\title{
Artin Transfer Patterns on Descendant Trees of Finite $p$-Groups
}

\author{
Daniel C. Mayer* \\ Naglergasse 53, 8010 Graz, Austria \\ Email: algebraic.number.theory@algebra.at
}

Received 25 November 2015; accepted 25 January 2016; published 29 January 2016

Copyright (C) 2016 by author and Scientific Research Publishing Inc.

This work is licensed under the Creative Commons Attribution International License (CC BY).

http://creativecommons.org/licenses/by/4.0/

(c) (i) Open Access

\section{Abstract}

Based on a thorough theory of the Artin transfer homomorphism $T_{G, H}: G \rightarrow H / H^{\prime}$ from a group $G$ to the abelianization $H / H^{\prime}$ of a subgroup $H \leq G$ of finite index $n=(G: H)$, and its connection with the permutation representation $G \rightarrow S_{n}$ and the monomial representation $G \rightarrow H<S_{n}$ of $G$, the Artin pattern $G \mapsto(\tau(G), \varkappa(G))$, which consists of families $\tau(G)=\left(H / H^{\prime}\right)_{H \leq G}, \quad$ resp. $\varkappa(G)=\left(\operatorname{ker}\left(T_{G, H}\right)\right)_{H \leq G}$, of transfer targets, resp. transfer kernels, is defined for the vertices $G \in \mathcal{T}$ of any descendant tree $\mathcal{T}$ of finite $p$-groups. It is endowed with partial order relations $\tau(\pi(G)) \leq \tau(G)$ and $\varkappa(\pi(G)) \geq \varkappa(G)$, which are compatible with the parent-descendant relation $\pi(G)<G$ of the edges $G \rightarrow \pi(G)$ of the tree T. The partial order enables termination criteria for the $p$-group generation algorithm which can be used for searching and identifying a finite $p$-group $G$, whose Artin pattern $(\tau(G), \varkappa(G))$ is known completely or at least partially, by constructing the descendant tree with the abelianization $G / G^{\prime}$ of $G$ as its root. An appendix summarizes details concerning induced homomorphisms between quotient groups, which play a crucial role in establishing the natural partial order on Artin patterns $(\tau(G), \varkappa(G))$ and explaining the stabilization, resp. polarization, of their components in descendant trees $\mathcal{T}$ of finite $p$-groups.

\section{Keywords}

Artin Transfer, Kernel Type, Target Type, Descendant Tree, Coclass Tree, Coclass Graph

\footnotetext{
${ }^{*}$ Respectfully dedicated to Professor M. F. Newman.
} 


\section{Introduction}

P 1.1. In the mathematical field of group theory, an Artin transfer is a certain homomorphism from an arbitrary finite or infinite group to the commutator quotient group of a subgroup of finite index.

Originally, such transfer mappings arose as group theoretic counterparts of class extension homomorphisms of abelian extensions of algebraic number fields by applying Artin's reciprocity isomorphism ([1], §4, Allgemeines Reziprozitätsgesetz, p. 361) to ideal class groups and analyzing the resulting homomorphisms between quotients of Galois groups ([2], §2, p. 50).

However, independently of number theoretic applications, a natural partial order on the kernels and targets of Artin transfers, has recently been found to be compatible with parent-child relations between finite $p$-groups, where $p$ denotes a prime number. Such ancestor-descendant relations can be visualized conveniently in descendant trees ([3], §4, pp. 163-164).

Consequently, Artin transfers provide valuable information for classifying finite $p$-groups by kernel-target patterns and for searching and identifying particular groups in descendant trees by looking for patterns defined by kernels and targets of Artin transfers. These strategies of pattern recognition are useful not only in purely group theoretic context, but also, most importantly, for applications in algebraic number theory concerning Galois groups of higher $p$-class fields and Hilbert $p$-class field towers. The reason is that the unramified extensions of a base field contain information in the shape of capitulation patterns and class group structures, and these arithmetic invariants can be translated into group theoretic data on transfer kernels and targets by means of Artin's reciprocity law of class field theory. The natural partial order on Artin patterns admits termination criteria for a search through a descendant tree with the aid of recursive executions of the $p$-group generation algorithm by Newman [4] and O'Brien [5].

$\mathbf{P}$ 1.2. The organization of this article is as follows. The detailed theory of the transfer will be developed in $\S \S$ 2 and 3, followed by computational implementations in $\S 4$. It is our intention to present more than the least common multiple of the original papers by Schur [6] and Artin [2] and the relevant sections of the text books by Hall [7], Huppert [8], Gorenstein [9], Aschbacher [10], Doerk and Hawkes [11], Smith and Tabachnikova [12], and Isaacs [13].

However, we shall not touch upon fusion and focal subgroups, which form the primary goal of the mentioned authors, except Artin. Our focus will rather be on a sound foundation of Artin patterns, consisting of families of transfer kernels and targets, and their stabilization, resp. polarization, in descendant trees of finite $p$-groups. These phenomena arise from a natural partial order on Artin patterns which is compatible with ancestordescendant relations in trees, and is established in its most general form in $\S \S 5$ and 6 .

Since our endeavour is to give the most general view of each partial result, we came to the conviction that categories, functors and natural transformations are the adequate tools for expressing the appropriate range of validity for the facts connected with the partial order relation on Artin patterns. Inspired by Bourbaki's method of exposition [14], Appendix on induced homomorphisms, which is separated to avoid a disruption of the flow of exposition, goes down to the origins exploiting set theoretic facts concerning direct images and inverse pre-images of mappings which are crucial for explaining the natural partial order of Artin patterns.

\section{Transversals and Their Permutations}

\subsection{Transversals of a Subgroup}

Let $G$ be a group and $H \leq G$ be a subgroup of finite index $n=(G: H) \geq 1$.

Definition 2.1. See also ([6], p. 1013); ([7], (1.5.1), p. 11); ([8], Satz 2.5, p. 5).

1). A left transversal of $H$ in $G$ is an ordered system $\left(\ell_{1}, \cdots, \ell_{n}\right)$ of representatives for the left cosets of $H$ in $G$ such that $G=\bigcup_{i=1}^{n} \ell_{i} H$ is a disjoint union.

2). Similarly, a right transversal of $H$ in $G$ is an ordered system $\left(r_{1}, \cdots, r_{n}\right)$ of representatives for the right cosets of $H$ in $G$ such that $G=\bigcup_{i=1}^{n} H r_{i}$ is a disjoint union.

Remark 2.1. For any transversal of $H$ in $G$, there exists a unique subscript $1 \leq i_{0} \leq n$ such that $\ell_{i_{0}} \in H$, resp. $r_{i_{0}} \in H$. The element $\ell_{i_{0}}$, resp. $r_{i_{0}}$, which represents the principal coset (i.e., the subgroup $H$ itself) may be replaced by the neutral element 1 .

Lemma 2.1. See also ([6], p. 1015); ([7], (1.5.2), p. 11); ([8], Satz 2.6, p. 6).

1). If $G$ is non-abelian and $H$ is not a normal subgroup of $G$, then we can only say that the inverse elements $\left(\ell_{1}^{-1}, \cdots, \ell_{n}^{-1}\right)$ of a left transversal $\left(\ell_{1}, \cdots, \ell_{n}\right)$ form a right transversal of $H$ in $G$. 
2). However, if $H \unlhd G$ is a normal subgroup of $G$, then any left transversal is also a right transversal of $H$ in $G$.

Proof. 1). Since the mapping $G \rightarrow G, x \mapsto x^{-1}$ is an involution, that is a bijection which is its own inverse, we see that

$$
G=\bigcup_{i=1}^{n} \ell_{i} H \text { implies } G=G^{-1}=\bigcup_{i=1}^{n}\left(\ell_{i} H\right)^{-1}=\bigcup_{i=1}^{n} H^{-1} \ell_{i}^{-1}=\bigcup_{i=1}^{n} H \ell_{i}^{-1} .
$$

2). For a normal subgroup $H \unlhd G$, we have $x H=H x$ for each $x \in G$.

Let $\phi: G \rightarrow T$ be a group homomorphism and $\left(\ell_{1}, \cdots, \ell_{n}\right)$ be a left transversal of a subgroup $H$ in $G$ with finite index $n=(G: H) \geq 1$. We must check whether the image of this transversal under the homomorphism is a transversal again.

Proposition 2.1. The following two conditions are equivalent.

1). $\left(\phi\left(\ell_{1}\right), \cdots, \phi\left(\ell_{n}\right)\right)$ is a left transversal of the subgroup $\phi(H)$ in the image $\operatorname{im}(\phi)=\phi(G)$ with finite index $(\phi(G): \phi(H))=n$.

2). $\operatorname{ker}(\phi) \leq H$.

We emphasize this important equivalence in a formula:

$$
\phi(G)=\bigcup_{i=1}^{n} \phi\left(\ell_{i}\right) \phi(H) \text { and }(\phi(G): \phi(H))=n \Leftrightarrow \operatorname{ker}(\phi) \leq H
$$

Proof. By assumption, we have the disjoint left coset decomposition $G=\bigcup_{i=1}^{n} \ell_{i} H$ which comprises two statements simultaneously.

Firstly, the group $G=\bigcup_{i=1}^{n} \ell_{i} H$ is a union of cosets,

and secondly, any two distinct cosets have an empty intersection $\ell_{i} H \cap \ell_{j} H=\varnothing$, for $i \neq j$.

Due to the properties of the set mapping associated with $\phi$, the homomorphism $\phi$ maps the union to another union

$$
\phi(G)=\phi\left(\bigcup_{i=1}^{n} \ell_{i} H\right)=\bigcup_{i=1}^{n} \phi\left(\ell_{i} H\right)=\bigcup_{i=1}^{n} \phi\left(\ell_{i}\right) \phi(H)
$$

but weakens the equality for the intersection to a trivial inclusion

$$
\varnothing=\phi(\varnothing)=\phi\left(\ell_{i} H \cap \ell_{j} H\right) \subseteq \phi\left(\ell_{i} H\right) \cap \phi\left(\ell_{j} H\right)=\phi\left(\ell_{i}\right) \phi(H) \cap \phi\left(\ell_{j}\right) \phi(H), \text { for } i \neq j .
$$

To show that the images of the cosets remain disjoint we need the property $\operatorname{ker}(\phi) \leq H$ of the homomorphism $\phi$.

Suppose that $\phi\left(\ell_{i}\right) \phi(H) \cap \phi\left(\ell_{j}\right) \phi(H) \neq \varnothing$ for some $1 \leq i \leq j \leq n$,

then we have $\phi\left(\ell_{i}\right) \phi\left(h_{i}\right)=\phi\left(\ell_{j}\right) \phi\left(h_{j}\right)$ for certain elements $h_{i}, h_{j} \in H$.

Multiplying by $\phi\left(\ell_{j}\right)^{-1}$ from the left and by $\phi\left(h_{j}\right)^{-1}$ from the right, we obtain

$$
\phi\left(\ell_{j}^{-1} \ell_{i} h_{i} h_{j}^{-1}\right)=\phi\left(\ell_{j}\right)^{-1} \phi\left(\ell_{i}\right) \phi\left(h_{i}\right) \phi\left(h_{j}\right)^{-1}=1 \text {, that is, } \ell_{j}^{-1} \ell_{i} h_{i} h_{j}^{-1} \in \operatorname{ker}(\phi) \leq H .
$$

Since $h_{i} h_{j}^{-1} \in H$, this implies $\ell_{j}^{-1} \ell_{i} \in H$, resp. $\ell_{i} H=\ell_{j} H$, and thus $i=j$. (This part of the proof is also covered by ([13], Thm. X. 21, p. 340) and, in the context of normal subgroups instead of homomorphisms, by ([7], Thm. 2.3.4, p. 29) and ([8], Satz 3.10, p. 16))

Conversely, we use contraposition.

If the kernel $\operatorname{ker}(\phi)$ of $\phi$ is not contained in the subgroup $H$, then there exists an element $x \in G \backslash H$ such that $\phi(x)=1$.

But then the homomorphism $\phi$ maps the disjoint cosets $x H \bigcap 1 \cdot H=\varnothing$

to equal cosets $\phi(x) \phi(H) \cap \phi(1) \phi(H)=1 \cdot \phi(H) \cap 1 \cdot \phi(H)=\phi(H)$.

\subsection{Permutation Representation}

P 2.1. Suppose $\left(\ell_{1}, \cdots, \ell_{n}\right)$ is a left transversal of a subgroup $H \leq G$ of finite index $n=(G: H) \geq 1$ in a group $\mathrm{G}$. A fixed element $x \in G$ gives rise to a unique permutation $\lambda_{x} \in S_{n}$ of the left cosets of $\mathrm{H}$ in $\mathrm{G}$ by left 
multiplication such that

$$
x \ell_{i} H=\ell_{\lambda_{x}(i)} H, \quad \text { resp. } \quad x \ell_{i} \in \ell_{\lambda_{x}(i)} H, \quad \text { resp. } \quad u_{x}(i):=\ell_{\lambda_{x}(i)}^{-1} x \ell_{i} \in H,
$$

for each $1 \leq i \leq n$.

Similarly, if $\left(r_{1}, \cdots, r_{n}\right)$ is a right transversal of $H$ in $G$, then a fixed element $x \in G$ gives rise to a unique permutation $\rho_{x} \in S_{n}$ of the right cosets of $H$ in $G$ by right multiplication such that

$$
H r_{i} x=H r_{\rho_{x}(i)} \text {, resp. } \quad r_{i} x \in H r_{\rho_{x}(i)} \text {, resp. } \quad w_{x}(i):=r_{i} x r_{\rho_{x}(i)}^{-1} \in H,
$$

for each $1 \leq i \leq n$.

The elements $u_{x}(i)$, resp. $w_{x}(i), 1 \leq i \leq n$, of the subgroup $H$ are called the monomials associated with $x$ with respect to $\left(\ell_{1}, \cdots, \ell_{n}\right)$, resp. $\left(r_{1}, \cdots, r_{n}\right)$.

Definition 2.2 See also ([8], Hauptsatz 6.2, p. 28).

The mapping $G \rightarrow S_{n}, x \mapsto \lambda_{x}$, resp. $G \rightarrow S_{n}, x \mapsto \rho_{x}$, is called the permutation representation of $G$ in $S_{n}$ with respect to $\left(\ell_{1}, \cdots, \ell_{n}\right)$, resp. $\left(r_{1}, \cdots, r_{n}\right)$.

Lemma 2.2. For the special right transversal $\left(\ell_{1}^{-1}, \cdots, \ell_{n}^{-1}\right)$ associated to the left transversal $\left(\ell_{1}, \cdots, \ell_{n}\right)$, we have the following relations between the monomials and permutations corresponding to an element $x \in G$ :

$$
w_{x^{-1}}(i)=u_{x}(i)^{-1} \text { for } 1 \leq i \leq n \text { and } \rho_{x^{-1}}=\lambda_{x} .
$$

Proof. For the right transversal $\left(\ell_{1}^{-1}, \cdots, \ell_{n}^{-1}\right)$, we have $w_{x}(i)=\ell_{i}^{-1} x \ell_{\rho_{x}(i)}$, for each $1 \leq i \leq n$.

On the other hand, for the left transversal $\left(\ell_{1}, \cdots, \ell_{n}\right)$, we have

$$
u_{x}(i)^{-1}=\left(\ell_{\lambda_{x}(i)}^{-1} x \ell_{i}\right)^{-1}=\ell_{i}^{-1} x^{-1} \ell_{\lambda_{x}(i)}=\ell_{i}^{-1} x^{-1} \ell_{\rho_{x^{-1}}(i)}=w_{x^{-1}}(i) \text {, for each } 1 \leq i \leq n .
$$

This relation simultaneously shows that, for any $x \in G$, the permutation representations and the associated monomials are connected by

$$
\rho_{x^{-1}}=\lambda_{x} \text { and } w_{x^{-1}}(i)=u_{x}(i)^{-1} \text {, }
$$

for each $1 \leq i \leq n$.

\section{Artin Transfer}

Let $G$ be a group and $H \leq G$ be a subgroup of finite index $n=(G: H) \geq 1$. Assume that $\left(\ell_{1}, \cdots, \ell_{n}\right)$, resp. $\left(r_{1}, \cdots, r_{n}\right)$, is a left, resp. right, transversal of $H$ in $G$ with associated permutation representation $G \rightarrow S_{n}$, $x \mapsto \lambda_{x}$, resp. $\rho_{x}$, such that $u_{x}(i):=\ell_{\lambda_{x}(i)}^{-1} \chi \ell_{i} \in H$, resp. $w_{x}(i):=r_{i} x r_{\rho_{x}(i)}^{-1} \in H$, for $1 \leq i \leq n$.

Definition 3.3. See also ([6], p. 1014); ([2], §2, p. 50); ([7], (14.2.2-4), p. 202); ([8], p. 413); ([9], p. 248); ([10], p. 197); ([11], Dfn.(17.1), p. 60); ([12], p. 154); ([13], p. 149); ([15], p. 2).

The Artin transfer $T_{G, H}: G \rightarrow H / H^{\prime}$ from $G$ to the abelianization $H / H^{\prime}$ of $H$ with respect to $\left(\ell_{1}, \cdots, \ell_{n}\right)$, resp. $\left(r_{1}, \cdots, r_{n}\right)$, is defined by

$$
T_{G, H}^{(\ell)}(x):=\prod_{i=1}^{n} \ell_{\lambda_{x}(i)}^{-1} x \ell_{i} \cdot H^{\prime} \quad \text { or briefly } \quad T_{G, H}(x)=\prod_{i=1}^{n} u_{x}(i) \cdot H^{\prime},
$$

resp.

for $x \in G$.

$$
T_{G, H}^{(r)}(x):=\prod_{i=1}^{n} r_{i} x r_{\rho_{x}(i)}^{-1} \cdot H^{\prime} \quad \text { or briefly } \quad T_{G, H}(x)=\prod_{i=1}^{n} w_{x}(i) \cdot H^{\prime}
$$

Remark 3.1. I.M. Isaacs [13], p. 149 calls the mapping $P: G \rightarrow H, \quad x \mapsto \prod_{i=1}^{n} u_{x}(i)$, resp. $x \mapsto \prod_{i=1}^{n} w_{x}(i)$, the pre-transfer from $\mathrm{G}$ to $\mathrm{H}$. The pre-transfer can be composed with a homomorphism $\phi: H \rightarrow A$ from $\mathrm{H}$ into an abelian group A to define a more general version of the transfer $(\phi \circ P): G \rightarrow A, \quad x \mapsto \prod_{i=1}^{n} \phi\left(u_{x}(i)\right)$, resp. $x \mapsto \prod_{i=1}^{n} \phi\left(w_{x}(i)\right)$, from G to A via $\phi$, which occurs in the book by D. Gorenstein ([9], p. 248). Taking the natural epimorphism $\phi: H \rightarrow H / H^{\prime}, \quad v \mapsto v H^{\prime}$, yields the Definition 3.3 of the Artin transfer $T_{G, H}$ in its 
original form by I. Schur ([6], p. 1014) and by E. Artin ([2], §2, p. 50), which has also been dubbed Verlagerung by H. Hasse ([16], §27.4, pp. 170-171). Note that, in general, the pre-transfer is neither independent of the transversal nor a group homomorphism.

\subsection{Independence of the Transversal}

Assume that $\left(g_{1}, \cdots, g_{n}\right)$ is another left transversal of $H$ in $G$ such that $G=\bigcup_{i=1}^{n} g_{i} H$.

Proposition 3.1. See also ([6], p. 1014); ([7], Thm. 14.2.1, p. 202); ([8], Hilfssatz 1.5, p. 414); ([9], Thm. 3.2, p. 246); ([10], (37.1), p.198); ([11], Thm.(17.2), p.61); ([12], p.154); ([13], Thm.5.1, p.149); ([15], Prop.2, p. 2).

The Artin transfers with respect to $(g)$ and $(\ell)$ coincide, $T_{G, H}^{(g)}=T_{G, H}^{(\ell)}$.

Proof. There exists a unique permutation $\sigma \in S_{n}$ such that $\ell_{i} H=g_{\sigma(i)} H$, for all $1 \leq i \leq n$. Consequently, $h_{i}:=\ell_{i}^{-1} g_{\sigma(i)} \in H$, resp. $g_{\sigma(i)}=\ell_{i} h_{i}$ with $h_{i} \in H$, for all $1 \leq i \leq n$. For a fixed element $x \in G$, there exists a unique permutation $\gamma_{x} \in S_{n}$ such that we have

$$
g_{\gamma_{x}(\sigma(i))} H=x g_{\sigma(i)} H=x \ell_{i} h_{i} H=x \ell_{i} H=\ell_{\lambda_{x}(i)} H=\ell_{\lambda_{x}(i)} h_{\lambda_{x}(i)} H=g_{\sigma\left(\lambda_{x}(i)\right)} H,
$$

for all $1 \leq i \leq n$. Therefore, the permutation representation of $G$ with respect to $\left(g_{1}, \cdots, g_{n}\right)$ is given by $\gamma_{x} \circ \sigma=\sigma \circ \lambda_{x}$, resp. $\gamma_{x}=\sigma \circ \lambda_{x} \circ \sigma^{-1} \in S_{n}$, for $x \in G$. Furthermore, for the connection between the elements $v_{x}(i):=g_{\gamma_{x}(i)}^{-1} x g_{i} \in H$ and $u_{x}(i):=\ell_{\lambda_{x}(i)}^{-1} x \ell_{i} \in H$, we obtain

$$
\begin{aligned}
v_{x}(\sigma(i)) & =g_{\gamma_{x}(\sigma(i))}^{-1} x g_{\sigma(i)}=g_{\sigma\left(\lambda_{x}(i)\right)}^{-1} x \ell_{i} h_{i}=\left(\ell_{\lambda_{x}(i)} h_{\lambda_{x}(i)}\right)^{-1} x \ell_{i} h_{i} \\
& =h_{\lambda_{x}(i)}^{-1} \ell_{\lambda_{x}(i)}^{-1} x \ell_{i} h_{i}=h_{\lambda_{x}(i)}^{-1} u_{x}(i) h_{i},
\end{aligned}
$$

for all $1 \leq i \leq n$. Finally, due to the commutativity of the quotient group $H / H^{\prime}$ and the fact that $\sigma$ and $\lambda_{x}$ are permutations, the Artin transfer turns out to be independent of the left transversal:

$$
\begin{aligned}
T_{G, H}^{(g)}(x) & =\prod_{i=1}^{n} v_{x}(\sigma(i)) \cdot H^{\prime}=\prod_{i=1}^{n} h_{\lambda_{x}(i)}^{-1} u_{x}(i) h_{i} \cdot H^{\prime}=\prod_{i=1}^{n} u_{x}(i) \prod_{i=1}^{n} h_{\lambda_{x}(i)}^{-1} \prod_{i=1}^{n} h_{i} \cdot H^{\prime} \\
& =\prod_{i=1}^{n} u_{x}(i) \cdot 1 \cdot H^{\prime}=\prod_{i=1}^{n} u_{x}(i) \cdot H^{\prime}=T_{G, H}^{(\ell)}(x),
\end{aligned}
$$

as prescribed in Definition 3.1, Equation (3.1).

It is clear that a similar proof shows that the Artin transfer is independent of the choice between two different right transversals. It remains to show that the Artin transfer with respect to a right transversal coincides with the Artin transfer with respect to a left transversal.

For this purpose, we select the special right transversal $\left(\ell_{1}^{-1}, \cdots, \ell_{n}^{-1}\right)$ associated to the left transversal $\left(\ell_{1}, \cdots, \ell_{n}\right)$, as explained in Lemma 2.1 and Lemma 2.2.

Proposition 3.2. The Artin transfers with respect to $\left(\ell^{-1}\right)$ and $(\ell)$ coincide, $T_{G, H}^{\left(\ell^{-1}\right)}=T_{G, H}^{(\ell)}$.

Proof. Using (2.4) in Lemma 2.2 and the commutativity of $H / H^{\prime}$, we consider the expression

$$
\begin{aligned}
T_{G, H}^{\left(\ell^{-1}\right)}(x) & =\prod_{i=1}^{n} \ell_{i}^{-1} x \ell_{\rho_{x}(i)} \cdot H^{\prime}=\prod_{i=1}^{n} w_{x}(i) \cdot H^{\prime}=\prod_{i=1}^{n} u_{x^{-1}}(i)^{-1} \cdot H^{\prime} \\
& =\left(\prod_{i=1}^{n} u_{x^{-1}}(i) \cdot H^{\prime}\right)^{-1}=\left(T_{G, H}^{(\ell)}\left(x^{-1}\right)\right)^{-1}=T_{G, H}^{(\ell)}(x) .
\end{aligned}
$$

The last step is justified by the fact that the Artin transfer is a homomorphism. This will be shown in the following subsection 3.2.

\subsection{Artin Transfers as Homomorphisms}

Let $\left(\ell_{1}, \cdots, \ell_{n}\right)$ be a left transversal of $H$ in $G$.

Theorem 3.1. See also ([6], p. 1014); ([7], Thm. 14.2.1, p. 202); ([8], Hauptsatz 1.4, p. 413); ([9], Thm. 3.2, p. 246); ([10], (37.2), p.198); ([11], Thm.(17.2), p.61); ([12], p. 155); ([13], Thm.5.2, p. 150); ([15], Prop.1, p. 2). 
The Artin transfer $T_{G, H}: G \rightarrow H / H^{\prime}, \quad x \mapsto \prod_{i=1}^{n} \ell_{\lambda_{x}(i)}^{-1} x \ell_{i} \cdot H^{\prime}$ and the permutation representation $G \rightarrow S_{n}, x \mapsto \lambda_{x}$ are group homomorphisms:

$$
T_{G, H}(x y)=T_{G, H}(x) \cdot T_{G, H}(y) \text { and } \lambda_{x y}=\lambda_{x} \circ \lambda_{y} \text { for } x, y \in G .
$$

Proof. Let $x, y \in G$ be two elements with transfer images $T_{G, H}(x)=\prod_{i=1}^{n} \ell_{\lambda_{x}(i)}^{-1} x \ell_{i} \cdot H^{\prime}$ and $T_{G, H}(y)=\prod_{j=1}^{n} \ell_{\lambda_{y}(j)}^{-1} y \ell_{j} \cdot H^{\prime}$. Since $H / H^{\prime}$ is abelian and $\lambda_{y}$ is a permutation, we can change the order of the factors in the following product:

$$
\begin{aligned}
& T_{G, H}(x) \cdot T_{G, H}(y)=\prod_{i=1}^{n} \ell_{\lambda_{x}(i)}^{-1} x \ell_{i} \cdot H^{\prime} \cdot \prod_{j=1}^{n} \ell_{\lambda_{y}(j)}^{-1} y \ell_{j} \cdot H^{\prime} \\
&=\prod_{j=1}^{n} \ell_{\lambda_{x}\left(\lambda_{y}(j)\right)}^{-1} x \ell_{\lambda_{y}(j)} \cdot H^{\prime} \cdot \prod_{j=1}^{n} \ell_{\lambda_{y}(j)}^{-1} y \ell_{j} \cdot H^{\prime} \\
&=\prod_{j=1}^{n} \ell_{\lambda_{x}\left(\lambda_{y}(j)\right)}^{-1} x \ell_{\lambda_{y}(j)} \ell_{\lambda_{y}(j)}^{-1} y \ell_{j} \cdot H^{\prime} \\
&=\prod_{j=1}^{n} \ell^{-1}\left(\left(\lambda_{x} \circ \lambda_{y}\right)(j)\right) \\
& x y \ell_{j} \cdot H^{\prime}=T_{G, H}(x y) .
\end{aligned}
$$

This relation simultaneously shows that the Artin transfer $T_{G, H}$ and the permutation representation $G \rightarrow S_{n}, x \mapsto \lambda_{x}$ are homomorphisms, since $T_{G, H}(x y)=T_{G, H}(x) \cdot T_{G, H}(y)$ and $\lambda_{x y}=\lambda_{x} \circ \lambda_{y}$, in a covariant way.

\subsection{Monomial Representation}

Let $\left(\ell_{1}, \cdots, \ell_{n}\right)$, resp. $\left(r_{1}, \cdots, r_{n}\right)$, be a left, resp. right, transversal of a subgroup $H$ in a group $G$. Using the monomials $u_{x}(i)$, resp. $w_{x}(i)$, associated with an element $x \in G$ according to Equation (2.2), resp. (2.3), we define the following maps.

Definition 3.2. The mapping $G \rightarrow H^{n} \times S_{n}, x \mapsto\left(u_{x}(1), \cdots, u_{x}(n) ; \lambda_{x}\right)$, respectively $G \rightarrow H^{n} \times S_{n}, x \mapsto\left(w_{x}(1), \cdots, w_{x}(n) ; \rho_{x}\right)$, is called the monomial representation of $G$ in $H^{n} \times S_{n}$ with respect to $\left(\ell_{1}, \cdots, \ell_{n}\right)$, resp. $\left(r_{1}, \cdots, r_{n}\right)$.

P 3.1. It is illuminating to restate the homomorphism property of the Artin transfer in terms of the monomial representation. The images of the factors $x, y$ are given by $T_{G, H}(x)=\prod_{i=1}^{n} u_{x}(i) \cdot H^{\prime}$ and

$T_{G, H}(y)=\prod_{j=1}^{n} u_{y}(j) \cdot H^{\prime}$. In the proof of Theorem 3.1, the image of the product $x y$ turned out to be $T_{G, H}(x y)=\prod_{j=1}^{n} \ell_{\lambda_{x}\left(\lambda_{y}(j)\right)}^{-1} x \ell_{\lambda_{y}(j)} \ell_{\lambda_{y}(j)}^{-1} y \ell_{j} \cdot H^{\prime}=\prod_{j=1}^{n} u_{x}\left(\lambda_{y}(j)\right) \cdot u_{y}(j) \cdot H^{\prime}$, which is a very peculiar law of composition discussed in more detail in the sequel.

The law reminds of the crossed homomorphisms $x \mapsto u_{x}$ in the first cohomology group $\mathrm{H}^{1}(G, M)$ of a $G$-module $M$, which have the property $u_{x y}=u_{x}^{y} \cdot u_{y}$, for $x, y \in G$.

These peculiar structures can also be interpreted by endowing the cartesian product $H^{n} \times S_{n}$ with a special law of composition known as the wreath product $H \succ S_{n}$ of the groups $H$ and $S_{n}$ with respect to the set $\{1, \cdots, n\}$.

Definition 3.3. For $x, y \in G$, the wreath product of the associated monomials and permutations is given by

$$
\begin{aligned}
& \left(u_{x}(1), \cdots, u_{x}(n) ; \lambda_{x}\right) \cdot\left(u_{y}(1), \cdots, u_{y}(n) ; \lambda_{y}\right) \\
& :=\left(u_{x}\left(\lambda_{y}(1)\right) \cdot u_{y}(1), \cdots, u_{x}\left(\lambda_{y}(n)\right) \cdot u_{y}(n) ; \lambda_{x} \circ \lambda_{y}\right) \\
& =\left(u_{x y}(1), \cdots, u_{x y}(n) ; \lambda_{x y}\right),
\end{aligned}
$$

Theorem 3.2. See also ([7], Thm.14.1, p. 200); ([8], Hauptsatz 1.4, p. 413).

This law of composition on $H^{n} \times S_{n}$ causes the monomial representation $G \rightarrow H \imath S_{n}$, $x \mapsto\left(u_{x}(1), \cdots, u_{x}(n) ; \lambda_{x}\right)$ also to be a homomorphism. In fact, it is a faithful representation, that is an injective 
homomorphism, also called a monomorphism or embedding, in contrast to the permutation representation.

Proof. The homomorphism property has been shown above already. For a homomorphism to be injective, it suffices to show the triviality of its kernel. The neutral element of the group $H^{n} \times S_{n}$ endowed with the wreath product is given by $(1, \cdots, 1 ; 1)$, where the last 1 means the identity permutation. If

$\left(u_{x}(1), \cdots, u_{x}(n) ; \lambda_{x}\right)=(1, \cdots, 1 ; 1)$, for some $x \in G$, then $\lambda_{x}=1$ and consequently $1=u_{x}(i)=g_{\lambda_{x}(i)}^{-1} x g_{i}=g_{i}^{-1} x g_{i}$, for all $1 \leq i \leq n$. Finally, an application of the inverse inner automorphism with $g_{i}$ yields $x=1$, as required for injectivity.

The permutation representation cannot be injective if $G$ is infinite or at least of an order bigger than $n$ !, the factorial of $n$.

Remark 3.2. Formula (3.4) is an example for the left-sided variant of the wreath product on $H^{n} \times S_{n}$. However, we point out that the wreath product with respect to a right transversal $\left(r_{1}, \cdots, r_{n}\right)$ of $\mathrm{H}$ in $\mathrm{G}$ appears in its right-sided variant

$$
\begin{aligned}
& \left(w_{x}(1), \cdots, w_{x}(n) ; \rho_{x}\right) \cdot\left(w_{y}(1), \cdots, w_{y}(n) ; \rho_{y}\right) \\
& :=\left(w_{x}(1) \cdot w_{y}\left(\rho_{x}(1)\right), \cdots, w_{x}(n) \cdot w_{y}\left(\rho_{x}(n)\right) ; \rho_{y} \circ \rho_{x}\right) \\
& =\left(w_{x y}(1), \cdots, w_{x y}(n) ; \rho_{x y}\right),
\end{aligned}
$$

which implies that the permutation representation $G \rightarrow S_{n}, x \mapsto \rho_{x}$ is a homomorphism with respect to the opposite law of composition $\rho_{x y}=\rho_{y} \circ \rho_{x}$ on $S_{n}$, in a contravariant manner.

It can be shown that the left-sided and the right-sided variant of the wreath product lead to isomorphic group structures on $H^{n} \times S_{n}$.

A related viewpoint is taken by M. Hall ([7], p. 200), who uses the multiplication of monomial matrices to describe the wreath product. Such a matrix can be represented in the form $M_{x}=\operatorname{diag}\left(w_{x}(1), \cdots, w_{x}(n)\right) \cdot P_{\rho_{x}}$ as the product of an invertible diagonal matrix over the group ring $K[H]$, where $K$ denotes a field, and the permutation matrix $P_{\rho_{x}}$ associated with the permutation $\rho_{x} \in S_{n}$. Multiplying two such monomial matrices yields a law of composition identical to the wreath product in the right-sided variant,

$$
\begin{aligned}
M_{x} \cdot M_{y} & =\operatorname{diag}\left(w_{x}(1), \cdots, w_{x}(n)\right) \cdot P_{\rho_{x}} \cdot \operatorname{diag}\left(w_{y}(1), \cdots, w_{y}(n)\right) \cdot P_{\rho_{y}} \\
& =\operatorname{diag}\left(w_{x}(1) \cdot w_{y}\left(\rho_{x}(1)\right), \cdots, w_{x}(n) \cdot w_{y}\left(\rho_{x}(n)\right)\right) \cdot P_{\rho_{x} \rho_{y}} .
\end{aligned}
$$

Whereas B. Huppert ([8], p. 413) uses the monomial representation for defining the Artin transfer by composition with the unsigned determinant, we prefer giving the immediate Definition 3.3 and merely illustrating the homomorphism property of the Artin transfer with the aid of the monomial representation.

\subsection{Composition of Artin Transfers}

Let $G$ be a group with nested subgroups $K \leq H \leq G$ such that the indices $(G: H)=n,(H: K)=m$ and $(G: K)=(G: H) \cdot(H: K)=n \cdot m$ are finite.

Theorem 3.3. See also ([7], Thm.14.2.1, p. 202); ([8], Satz 1.6, p. 415); ([11], Lem.(17.3), p. 61); ([13], Thm.10.8, p. 301); ([15], Prop.3, p. 3).

Then the Artin transfer $T_{G, K}$ is the compositum of the induced transfer $\tilde{T}_{H, K}: H / H^{\prime} \rightarrow K / K^{\prime}$ (in the sense of Corollary 7.1 or Corollary 7.3 in the Appendix) and the Artin transfer $T_{G, H}$, i.e.,

$$
T_{G, K}=\tilde{T}_{H, K} \circ T_{G, H} .
$$

This can be seen in the following manner.

Proof. If $\left(\ell_{1}, \cdots, \ell_{n}\right)$ is a left transversal of $H$ in $G$ and $\left(h_{1}, \cdots, h_{m}\right)$ is a left transversal of $K$ in $H$, that is $G=\bigcup_{i=1}^{n} \ell_{i} H$ and $H=\bigcup_{j=1}^{m} h_{j} K$, then $G=\bigcup_{i=1}^{n} \bigcup_{j=1}^{m} \ell_{i} h_{j} K$ is a disjoint left coset decomposition of $G$ with respect to $K$. See also ([7], Thm.1.5.3, p. 12); ([8], Satz 2.6, p. 6). Given two elements $x \in G$ and $y \in H$, there exist unique permutations $\lambda_{x} \in S_{n}$, and $\sigma_{y} \in S_{m}$, such that the associated monomials are given by

$u_{x}(i):=\ell_{\lambda_{x}(i)}^{-1} x \ell_{i} \in H$, for each $1 \leq i \leq n$, and $v_{y}(j):=h_{\sigma_{y}(j)}^{-1} y h_{j} \in K$, for each $1 \leq j \leq m$.

Then, using Corollary 7.3, we have 


$$
T_{G, H}(x)=\prod_{i=1}^{n} u_{x}(i) \cdot H^{\prime} \text {, and } \tilde{T}_{H, K}\left(y \cdot H^{\prime}\right)=T_{H, K}(y)=\prod_{j=1}^{m} v_{y}(j) \cdot K^{\prime} .
$$

For each pair of subscripts $1 \leq i \leq n$ and $1 \leq j \leq m$, we put $y_{i}:=u_{x}(i) \in H$ and obtain

$$
\begin{aligned}
x \ell_{i} h_{j} & =\ell_{\lambda_{x}(i)} \ell_{\lambda_{x}(i)}^{-1} x \ell_{i} h_{j}=\ell_{\lambda_{x}(i)} u_{x}(i) h_{j}=\ell_{\lambda_{x}(i)} y_{i} h_{j} \\
& =\ell_{\lambda_{x}(i)} h_{\sigma_{y_{i}}(j)} h_{\sigma_{y_{i}}(j)}^{-1} y_{i} h_{j}=\ell_{\lambda_{x}(i)} h_{\sigma_{y_{i}}(j)} v_{y_{i}}(j),
\end{aligned}
$$

resp. $h_{\sigma_{y_{i}}(j)}^{-1} \ell_{\lambda_{x}(i)}^{-1} x \ell_{i} h_{j}=v_{y_{i}}(j)$. Thus, the image of $x$ under the Artin transfer $T_{G, K}$ is given by

$$
\begin{aligned}
T_{G, K}(x) & =\prod_{i=1}^{n} \prod_{j=1}^{m} v_{y_{i}}(j) \cdot K^{\prime}=\prod_{i=1}^{n} \prod_{j=1}^{m} h_{\sigma_{y_{i}}(j)}^{-1} \ell_{\lambda_{x}(i)}^{-1} x \ell_{i} h_{j} \cdot K^{\prime} \\
& =\prod_{i=1}^{n} \prod_{j=1}^{m} h_{\sigma_{y_{i}}(j)}^{-1} u_{x}(i) h_{j} \cdot K^{\prime}=\prod_{i=1}^{n} \prod_{j=1}^{m} h_{\sigma_{y_{i}}(j)}^{-1} y_{i} h_{j} \cdot K^{\prime}=\prod_{i=1}^{n} \tilde{T}_{H, K}\left(y_{i} \cdot H^{\prime}\right) \\
& =\tilde{T}_{H, K}\left(\prod_{i=1}^{n} y_{i} \cdot H^{\prime}\right)=\tilde{T}_{H, K}\left(\prod_{i=1}^{n} u_{x}(i) \cdot H^{\prime}\right)=\tilde{T}_{H, K}\left(T_{G, H}(x)\right) .
\end{aligned}
$$

\subsection{Wreath Product of $S_{m}$ and $S_{n}$}

P 3.2. Motivated by the proof of Theorem 3.3, we want to emphasize the structural peculiarity of the monomial representation

$$
G \rightarrow K^{n \cdot m} \times S_{n \cdot m}, x \mapsto\left(k_{x}(1,1), \cdots, k_{x}(n, m) ; \gamma_{x}\right),
$$

which corresponds to the compositum of Artin transfers, defining

$$
k_{x}(i, j):=\left((\ell h)_{\gamma_{x}(i, j)}\right)^{-1} x(\ell h)_{(i, j)} \in K
$$

for a permutation $\gamma_{x} \in S_{n \cdot m}$, and using the symbolic notation $(\ell h)_{(i, j)}:=\ell_{i} h_{j}$ for all pairs of subscripts $1 \leq i \leq n, 1 \leq j \leq m$.

The preceding proof has shown that $k_{x}(i, j)=h_{\sigma_{y_{i}}(j)}^{-1} \ell_{\lambda_{x}(i)}^{-1} x \ell_{i} h_{j}$. Therefore, the action of the permutation $\gamma_{x}$ on the set $1, n \times 1, m$ is given by $\gamma_{x}(i, j)=\left(\lambda_{x}(i), \sigma_{u_{x}(i)}(j)\right)$. The action on the second component $j$ depends on the first component $i$ (via the permutation $\sigma_{u_{x}(i)} \in S_{m}$ ), whereas the action on the first component $i$ is independent of the second component $j$. Therefore, the permutation $\gamma_{x} \in S_{n \cdot m}$ can be identified with the multiplet $\left(\lambda_{x} ; \sigma_{u_{x}(1)}, \cdots, \sigma_{u_{x}(n)}\right) \in S_{n} \times S_{m}^{n}$, which will be written in twisted form in the sequel.

The permutations $\gamma_{x}$, which arise as second components of the monomial representation

$$
G \rightarrow K 々 S_{n \cdot m}, \mapsto\left(k_{x}(1,1), \cdots, k_{x}(n, m) ; \gamma_{x}\right),
$$

are of a very special kind. They belong to the stabilizer of the natural equipartition of the set $[1, n] \times[1, m]$ into the $n$ rows of the corresponding matrix (rectangular array). Using the peculiarities of the composition of Artin transfers in the previous section, we show that this stabilizer is isomorphic to the wreath product $S_{m}<S_{n}$ of the symmetric groups $S_{m}$ and $S_{n}$ with respect to $\{1, \cdots, n\}$, whose underlying set $S_{m}^{n} \times S_{n}$ is endowed with the following law of composition in the left-sided variant.

$$
\begin{aligned}
\gamma_{x} \cdot \gamma_{z} & =\left(\sigma_{u_{x}(1)}, \cdots, \sigma_{u_{x}(n)} ; \lambda_{x}\right) \cdot\left(\sigma_{u_{z}(1)}, \cdots, \sigma_{u_{z}(n)} ; \lambda_{z}\right) \\
& =\left(\sigma_{u_{x}\left(\lambda_{z}(1)\right)} \circ \sigma_{u_{z}(1)}, \cdots, \sigma_{u_{x}\left(\lambda_{z}(n)\right)} \circ \sigma_{u_{z}(n)} ; \lambda_{x} \circ \lambda_{z}\right) \\
& =\left(\sigma_{u_{x z}(1)}, \cdots, \sigma_{u_{x z}(n)} ; \lambda_{x z}\right)=\gamma_{x z}
\end{aligned}
$$

for all $x, z \in G$.

This law reminds of the chain rule $D(g \circ f)(x)=D(g)(f(x)) \circ D(f)(x)$ for the Fréchet derivative in $x \in E$ of the compositum of differentiable functions $f: E \rightarrow F$ and $g: F \rightarrow G$ between complete normed spaces. 
The above considerations establish a third representation, the stabilizer representation,

$$
G \rightarrow S_{m} \prec S_{n}, x \mapsto\left(\sigma_{u_{x}(1)}, \cdots, \sigma_{u_{x}(n)} ; \lambda_{x}\right)
$$

of the group $G$ in the wreath product $S_{m} \prec S_{n}$, similar to the permutation representation and the monomial representation. As opposed to the latter, the stabilizer representation cannot be injective, in general. For instance, certainly not, if $G$ is infinite.

Formula (3.7) proves the following statement.

Theorem 3.4. The stabilizer representation $G \rightarrow S_{m} \prec S_{n}, x \mapsto \gamma_{x}=\left(\sigma_{u_{x}(1)}, \cdots, \sigma_{u_{x}(n)} ; \lambda_{x}\right)$ of the group $G$ in the wreath product $S_{m}\left\{S_{n}\right.$ of symmetric groups is a group homomorphism.

\subsection{Cycle Decomposition}

Let $\left(\ell_{1}, \cdots, \ell_{n}\right)$ be a left transversal of a subgroup $H \leq G$ of finite index $n=(G: H) \geq 1$ in a group $G$. Suppose the element $x \in G$ gives rise to the permutation $\lambda_{x} \in S_{n}$ of the left cosets of $H$ in $G$ such that $x \ell_{i} H=\ell_{\lambda_{x}(i)} H$, resp. $\ell_{\lambda_{x}(i)}^{-1} x \ell_{i}=: u_{x}(i) \in H$, for each $1 \leq i \leq n$.

Theorem 3.5. See also ([2], §2, p. 50); ([16], §27.4, p. 170); ([8], Hilfssatz 1.7, p. 415); ([9], Thm.3.3, p. 249); ([10], (37.3), p. 198); ([12], p. 154); ([13], Lem.5.5, p. 153); ([15], p. 5).

If the permutation $\lambda_{x}$ has the decomposition $\lambda_{x}=\prod_{j=1}^{t} \zeta_{j}$ into pairwise disjoint (and thus commuting) cycles $\zeta_{j} \in S_{n}$ of lengths $f_{j} \geq 1$, which is unique up to the ordering of the cycles, more explicitly, if

$$
\left(\ell_{j} H, \ell_{\zeta_{j}(j)} H, \ell_{\zeta_{j}^{2}(j)} H, \cdots, \ell_{\zeta_{j}^{f_{j}-1}(j)} H\right)=\left(\ell_{j} H, x \ell_{j} H, x^{2} \ell_{j} H, \cdots, x^{f_{j}-1} \ell_{j} H\right),
$$

for $1 \leq j \leq t$, and $\sum_{j=1}^{t} f_{j}=n$, then the image of $x \in G$ under the Artin transfer $T_{G, H}$ is given by

$$
T_{G, H}(x)=\prod_{j=1}^{t} \ell_{j}^{-1} x^{f_{j}} \ell_{j} \cdot H^{\prime} .
$$

Proof. The reason for this fact is that we obtain another left transversal of $H$ in $G$ by putting $g_{j, k}:=x^{k} \ell_{j}$ for $0 \leq k \leq f_{j}-1$ and $1 \leq j \leq t$, since

$$
G=\bigcup_{j=1}^{t} \bigcup_{k=0}^{f_{j}-1} x^{k} \ell_{j} H
$$

is a disjoint decomposition of $G$ into left cosets of $H$.

Let us fix a value of $1 \leq j \leq t$. For $0 \leq k \leq f_{j}-2$, we have

$$
x g_{j, k}=x x^{k} \ell_{j}=x^{k+1} \ell_{j}=g_{j, k+1} \in g_{j, k+1} H, \text { resp. } u_{x}(j, k):=g_{j, k+1}^{-1} x g_{j, k}=1 \in H .
$$

However, for $k=f_{j}-1$, we obtain

$$
x g_{j, f_{j}-1}=x x^{f_{j}-1} \ell_{j}=x^{f_{j}} \ell_{j} \in \ell_{j} H=g_{j, 0} H \text {, resp. } u_{x}\left(j, f_{j}-1\right):=g_{j, 0}^{-1} x g_{j, f_{j}-1}=\ell_{j}^{-1} x^{f_{j}} \ell_{j} \in H .
$$

Consequently,

$$
T_{G, H}(x)=\prod_{j=1}^{t} \prod_{k=0}^{f_{j}-1} u_{x}(j, k) \cdot H^{\prime}=\prod_{j=1}^{t}\left(\prod_{k=0}^{f_{j}-2} 1\right) \cdot u_{x}\left(j, f_{j}-1\right) \cdot H^{\prime}=\prod_{j=1}^{t} \ell_{j}^{-1} x^{f_{j}} \ell_{j} \cdot H^{\prime} .
$$

P 3.3. The cycle decomposition corresponds to a double coset decomposition $G=\bigcup_{j=1}^{t}\langle x\rangle \ell_{j} H$ of the group $\mathrm{G}$ modulo the cyclic group $\langle x\rangle$ and modulo the subgroup $\mathrm{H}$. It was actually this cycle decomposition form of the transfer homomorphism which was given by E. Artin in his original 1929 paper ([2], §2, p. 50).

\subsection{Transfer to a Normal Subgroup}

P 3.4. Now let $H \unlhd G$ be a normal subgroup of finite index $n=(G: H) \geq 1$ in a group G. Then we have 
$x H=H x$, for all $x \in G$, and there exists the quotient group $G / H$ of order n. For an element $x \in G$, we let $f:=\operatorname{ord}(x H)$ denote the order of the coset $x H$ in $G / H$, and we let $\left(\ell_{1}, \cdots, \ell_{t}\right)$ be a left transversal of the subgroup $\langle x, H\rangle$ in $\mathrm{G}$, where $t=n / f$.

Theorem 3.6. See also ([16], §27.4, VII, p. 171).

Then the image of $x \in G$ under the Artin transfer $T_{G, H}$ is given by

$$
T_{G, H}(x)=\prod_{j=1}^{t} \ell_{j}^{-1} x^{f} \ell_{j} \cdot H^{\prime} .
$$

Proof. $\langle x H\rangle$ is a cyclic subgroup of order $f$ in $G / H$, and a left transversal $\left(\ell_{1}, \cdots, \ell_{t}\right)$ of the subgroup $\langle x, H\rangle$ in $G$, where $t=n / f$ and $G=\bigcup_{j=1}^{t} \ell_{j}\langle x, H\rangle$ is the corresponding disjoint left coset decomposition, can be refined to a left transversal $\ell_{j} x^{k} \quad(1 \leq j \leq t, 0 \leq k \leq f-1)$ with disjoint left coset decomposition

$$
G=\bigcup_{j=1}^{t} \bigcup_{k=0}^{f-1} \ell_{j} x^{k} H
$$

of $H$ in $G$. Hence, the formula for the image of $x$ under the Artin transfer $T_{G, H}$ in the previous section takes the particular shape

$$
T_{G, H}(x)=\prod_{j=1}^{t} \ell_{j}^{-1} x^{f} \ell_{j} \cdot H^{\prime}
$$

with exponent $f$ independent of $j$.

Corollary 3.1. See also ([13], Lem.10.6, p. 300) for a special case.

In particular, the inner transfer of an element $x \in H$ is given as a symbolic power

$$
T_{G, H}(x)=x^{\operatorname{Tr}_{G}(H)} \cdot H^{\prime}
$$

with the trace element

$$
\operatorname{Tr}_{G}(H)=\sum_{j=1}^{t} \ell_{j} \in \mathbb{Z}[G]
$$

of $H$ in $G$ as symbolic exponent.

The other extreme is the outer transfer of an element $x \in G \backslash H$ which generates $G$ modulo $H$, that is $G=\langle x, H\rangle$. It is simply an $n$th power

$$
T_{G, H}(x)=x^{n} \cdot H^{\prime} .
$$

Proof. The inner transfer of an element $x \in H$, whose coset $x H=H$ is the principal set in $G / H$ of order $f=1$, is given as the symbolic power

$$
T_{G, H}(x)=\prod_{j=1}^{t} \ell_{j}^{-1} x \ell_{j} \cdot H^{\prime}=\prod_{j=1}^{t} x^{\ell_{j}} \cdot H^{\prime}=x^{\sum_{j=1}^{t} \ell_{j}} \cdot H^{\prime}
$$

with the trace element

$$
\operatorname{Tr}_{G}(H)=\sum_{j=1}^{t} \ell_{j} \in \mathbb{Z}[G]
$$

of $H$ in $G$ as symbolic exponent.

The outer transfer of an element $x \in G \backslash H$ which generates $G$ modulo $H$, that is $G=\langle x, H\rangle$, whose coset $x H$ is generator of $G / H$ with order $f=n$, is given as the $n$th power

$$
T_{G, H}(x)=\prod_{j=1}^{1} 1^{-1} \cdot x^{n} \cdot 1 \cdot H^{\prime}=x^{n} \cdot H^{\prime} .
$$

P 3.5. Transfers to normal subgroups will be the most important cases in the sequel, since the central concept of this article, the Artin pattern, which endows descendant trees with additional structure, consists of targets and kernels (§5) of Artin transfers from a group $G$ to intermediate groups $G^{\prime} \leq H \leq G$ between $G$ and its commutator subgroup $G^{\prime}$. For these intermediate groups we have the following lemma.

Lemma 3.1. All subgroups $H \leq G$ of a group $G$ which contain the commutator subgroup $G^{\prime}$ are normal 
subgroups $H \unlhd G$.

Proof. Let $G^{\prime} \leq H \leq G$. If $H$ were not a normal subgroup of $G$, then we had $x^{-1} H x \nsubseteq H$ for some element $x \in G \backslash H$. This would imply the existence of elements $h \in H$ and $y \in G \backslash H$ such that $x^{-1} h x=y$, and consequently the commutator $[h, x]=h^{-1} x^{-1} h x=h^{-1} y$ would be an element in $G \backslash H$ in contradiction to $G^{\prime} \leq H$.

Explicit implementations of Artin transfers in the simplest situations are presented in the following section.

\section{Computational Implementation}

\subsection{Abelianization of Type $(p, p)$}

P 4.1. Let $\mathrm{G}$ be a pro-p group with abelianization $G / G^{\prime}$ of elementary abelian type $(p, p)$. Then $\mathrm{G}$ has $p+1$ maximal subgroups $H_{i}<G \quad(1 \leq i \leq p+1)$ of index $\left(G: H_{i}\right)=p$. In this particular case, the Frattini subgroup $\Phi(G):=\bigcap_{i=1}^{p+1} H_{i}$, which is defined as the intersection of all maximal subgroups, coincides with the commutator subgroup $G^{\prime}=[G, G]$, since the latter contains all pth powers $G^{\prime} \geq G^{p}$, and thus we have $\Phi(G)=G^{p} \cdot G^{\prime}=G^{\prime}$.

For each $1 \leq i \leq p+1$, let $T_{i}: G \rightarrow H_{i} / H_{i}^{\prime}$ be the Artin transfer homomorphism from $G$ to the abelianization of $H_{i}$. According to Burnside's basis theorem, the group $G$ has generator rank $d(G)=2$ and can therefore be generated as $G=\langle x, y\rangle$ by two elements $x, y$ such that $x^{p}, y^{p} \in G^{\prime}$. For each of the normal subgroups $H_{i} \triangleleft G$, we need a generator $h_{i}$ with respect to $G^{\prime}$, and a generator $t_{i}$ of a transversal $\left(1, t_{i}, t_{i}^{2}, \cdots, t_{i}^{p-1}\right)$ such that $H_{i}=\left\langle h_{i}, G^{\prime}\right\rangle$ and $G=\left\langle t_{i}, H_{i}\right\rangle=\bigcup_{j=0}^{p-1} t_{i}^{j} H_{i}$.

A convenient selection is given by

$$
h_{1}=y, t_{1}=x \text {, and } h_{i}=x y^{i-2}, t_{i}=y, \text { for all } 2 \leq i \leq p+1 .
$$

Then, for each $1 \leq i \leq p+1$, it is possible to implement the inner transfer by

$$
T_{i}\left(h_{i}\right)=h_{i}^{\mathrm{Tr}_{G}\left(H_{i}\right)} \cdot H_{i}^{\prime}=h_{i}^{1+t_{i}+t_{i}^{2}+\cdots+t_{i}^{p-1}} \cdot H_{i}^{\prime},
$$

according to Equation (3.13) of Corollary 3.1, which can also be expressed by a product of two $p$ th powers,

$$
T_{i}\left(h_{i}\right)=h_{i} \cdot t_{i}^{-1} h_{i} t_{i} \cdot t_{i}^{-2} h_{i} t_{i}^{2} \cdots t_{i}^{-p+1} h_{i} t_{i}^{p-1} \cdot H_{i}^{\prime}=\left(h_{i} t_{i}^{-1}\right)^{p} t_{i}^{p} \cdot H_{i}^{\prime},
$$

and to implement the outer transfer as a complete $p$ th power by

$$
T_{i}\left(t_{i}\right)=t_{i}^{p} \cdot H_{i}^{\prime},
$$

according to Equation (3.15) of Corollary 3.1. The reason is that $\operatorname{ord}\left(h_{i} H_{i}\right)=1$ and $\operatorname{ord}\left(t_{i} H_{i}\right)=p$ in the quotient group $G / H_{i}$.

It should be pointed out that the complete specification of the Artin transfers $T_{i}$ also requires explicit knowledge of the derived subgroups $H_{i}^{\prime}$. Since $G^{\prime}$ is a normal subgroup of index $p$ in $H_{i}$, a certain general reduction is possible by $H_{i}^{\prime}=\left[H_{i}, H_{i}\right]=\left[G^{\prime}, H_{i}\right]=\left(G^{\prime}\right)^{h_{i}-1}$ ([17], Lem.2.1, p. 52), but an explicit pro-p presentation of $G$ must be known for determining generators of $G^{\prime}=\left\langle s_{1}, \cdots, s_{n}\right\rangle$, whence

$$
H_{i}^{\prime}=\left(G^{\prime}\right)^{h_{i}-1}=\left\langle\left[s_{1}, h_{i}\right], \cdots,\left[s_{n}, h_{i}\right]\right\rangle .
$$

\subsection{Abelianization of Type $\left(p^{2}, p\right)$}

P 4.2. Let $\mathrm{G}$ be a pro-p group with abelianization $G / G^{\prime}$ of non-elementary abelian type $\left(p^{2}, p\right)$. Then $\mathrm{G}$ has $p+1$ maximal subgroups $H_{1, i}<G \quad(1 \leq i \leq p+1)$ of index $\left(G: H_{1, i}\right)=p$ and $p+1$ subgroups $H_{2, i}<G$ $(1 \leq i \leq p+1)$ of index $\left(G: H_{2, i}\right)=p^{2}$.

Figure 1 visualizes this smallest non-trivial example of a multi-layered abelianization $G / G^{\prime}$ ([18], Dfn.3.13, p. 288).

For each $1 \leq i \leq p+1$, let $T_{1, i}: G \rightarrow H_{1, i} / H_{1, i}^{\prime}$, resp. $T_{2, i}: G \rightarrow H_{2, i} / H_{2, i}^{\prime}$, be the Artin transfer homomorphism from $G$ to the abelianization of $H_{1, i}$, resp. $H_{2, i}$. Burnside's basis theorem asserts that the group $G$ has generator rank $d(G)=2$ and can therefore be generated as $G=\langle x, y\rangle$ by two elements $x, y$ such that 
Order $p^{n}$
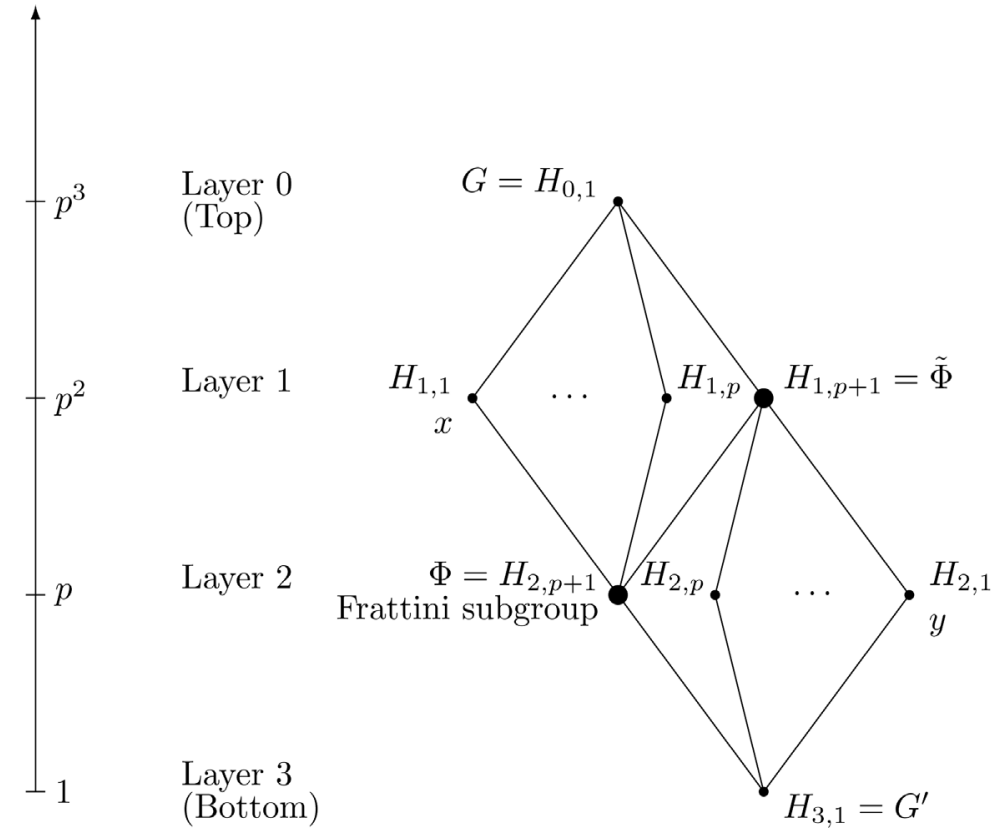

$\operatorname{Lyr}_{0}(G)=\left\{H_{0,1}\right\}$

$\operatorname{Lyr}_{1}(G)=\left\{H_{1,1}, \ldots, H_{1, p+1}\right\}$

Maximal subgroups

$\operatorname{Lyr}_{2}(G)=\left\{H_{2,1}, \ldots, H_{2, p+1}\right\}$

$\operatorname{Lyr}_{3}(G)=\left\{H_{3,1}\right\}$

Commutator subgroup

Figure 1. Layers of subgroups $G^{\prime} \leq H_{i, j} \leq G$ for $G / G^{\prime}=\left\langle x, y, G^{\prime}\right\rangle \simeq\left(p^{2}, p\right)$.

$x^{p^{2}}, y^{p} \in G^{\prime}$.

We begin by considering the first layer of subgroups. For each of the normal subgroups $H_{1, i} \triangleleft G$ $(1 \leq i \leq p)$, we select a generator

$$
h_{i}=x y^{i-1} \text { such that } H_{1, i}=\left\langle h_{i}, G^{\prime}\right\rangle .
$$

These are the cases where the factor group $H_{1, i} / G^{\prime}$ is cyclic of order $p^{2}$. However, for the distinguished maximal subgroup $H_{1, p+1}$, for which the factor group $H_{1, p+1} / G^{\prime}$ is bicyclic of type $(p, p)$, we need two generators

$$
h_{p+1}=y, h_{0}=x^{p} \text { such that } H_{1, p+1}=\left\langle h_{p+1}, h_{0}, G^{\prime}\right\rangle .
$$

Further, a generator $t_{i}$ of a transversal must be given such that $G=\left\langle t_{i}, H_{1, i}\right\rangle$, for each $1 \leq i \leq p+1$. It is convenient to define

$$
t_{i}=y \text {, for } 1 \leq i \leq p \text {, and } t_{p+1}=x .
$$

Then, for each $1 \leq i \leq p+1$, we have the inner transfer

$$
T_{1, i}\left(h_{i}\right)=h_{i}^{\mathrm{Tr}_{G}\left(H_{1, i}\right)} \cdot H_{1, i}^{\prime}=h_{i}^{1+t_{i}+t_{i}^{2}+\cdots+t_{i}^{p-1}} \cdot H_{1, i}^{\prime},
$$

which equals $\left(h_{i} t_{i}^{-1}\right)^{p} t_{i}^{p} \cdot H_{1, i}^{\prime}$, and the outer transfer

$$
T_{1, i}\left(t_{i}\right)=t_{i}^{p} \cdot H_{1, i}^{\prime}
$$

since $\operatorname{ord}\left(h_{i} H_{1, i}\right)=1$ and $\operatorname{ord}\left(t_{i} H_{1, i}\right)=p$.

Now we continue by considering the second layer of subgroups. For each of the normal subgroups $H_{2, i} \triangleleft G$ $(1 \leq i \leq p+1)$, we select a generator

$$
u_{1}=y, u_{i}=x^{p} y^{i-1} \text { for } 2 \leq i \leq p \text {, and } u_{p+1}=x^{p},
$$

such that $H_{2, i}=\left\langle u_{i}, G^{\prime}\right\rangle$. Among these subgroups, the Frattini subgroup $H_{2, p+1}=\left\langle x^{p}, G^{\prime}\right\rangle=G^{p} \cdot G^{\prime}$ is par- 
ticularly distinguished. A uniform way of defining generators $t_{i}, w_{i}$ of a transversal such that $G=\left\langle t_{i}, w_{i}, H_{2, i}\right\rangle$, is to set

$$
t_{i}=x, w_{i}=x^{p} \text {, for } 1 \leq i \leq p \text {, and } t_{p+1}=x, w_{p+1}=y \text {. }
$$

Since $\operatorname{ord}\left(u_{i} H_{2, i}\right)=1$, but on the other hand $\operatorname{ord}\left(t_{i} H_{2, i}\right)=p^{2}$ and $\operatorname{ord}\left(w_{i} H_{2, i}\right)=p$, for $1 \leq i \leq p+1$, with the single exception that $\operatorname{ord}\left(t_{p+1} H_{2, p+1}\right)=p$, we obtain the following expressions for the inner transfer

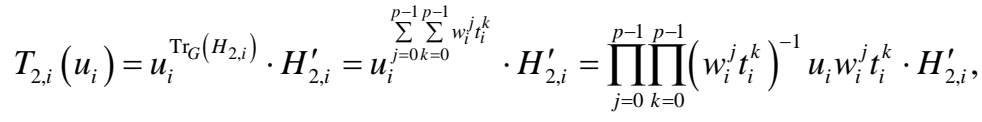

and for the outer transfer

$$
T_{2, i}\left(t_{i}\right)=t_{i}^{p^{2}} \cdot H_{2, i}
$$

exceptionally

$$
T_{2, p+1}\left(t_{p+1}\right)=\left(t_{p+1}^{p}\right)^{1+w_{p+1}+w_{p+1}^{2}+\cdots+w_{p+1}^{p-1}} \cdot H_{2, p+1}^{\prime}
$$

and

$$
T_{2, i}\left(w_{i}\right)=\left(w_{i}^{p}\right)^{1+t_{i}+t_{i}^{2}+\cdots+t_{i}^{p-1}} \cdot H_{2, i}^{\prime},
$$

for $1 \leq i \leq p+1$. Again, it should be emphasized that the structure of the derived subgroups $H_{1, i}^{\prime}$ and $H_{2, i}^{\prime}$ must be known explicitly to specify the action of the Artin transfers completely.

\section{Transfer Kernels and Targets}

P 5.1. After our thorough treatment of the general theory of Artin transfers in $\S \S 2$ and 3 , and their computational implementation for some simple cases in $\S 4$, we are now in the position to introduce Artin transfer patterns, which form the central concept of this article. They provide an incredibly powerful tool for classifying finite and infinite pro-p groups and for identifying a finite p-group $\mathrm{G}$ with sufficiently many assigned components of its Artin pattern by the strategy of pattern recognition. This is done in a search through the descendant tree with root $G / G^{\prime}$ by means of recursive applications of the p-group generation algorithm by Newman [4] and O'Brien [5].

An Artin transfer pattern consists of two families of transfer targets, resp. kernels, which are also called multiplets, whereas their individual components are referred to as singulets.

\subsection{Singulets of Transfer Targets}

Theorem 5.1. Let $G$ and $T$ be groups. Suppose that $H=\operatorname{im}(\varphi)=\varphi(G) \leq T$ is the image of $G$ under a homomorphism $\varphi: G \rightarrow T$, and $V=\varphi(U)$ is the image of an arbitrary subgroup $U \leq G$. Then the following claims hold without any further necessary assumptions.

1) The commutator subgroup of $V$ is the image of the commutator subgroup of $U$, that is

$$
V^{\prime}=\varphi\left(U^{\prime}\right) \text {. }
$$

2) The restriction $\left.\varphi\right|_{U}: U \rightarrow V$ is an epimorphism which induces a unique epimorphism

$$
\tilde{\varphi}: U / U^{\prime} \rightarrow V / V^{\prime}, x U^{\prime} \mapsto \varphi(x) V^{\prime} .
$$

Thus, the abelianization of $V$,

$$
V / V^{\prime} \simeq\left(U / U^{\prime}\right) / \operatorname{ker}(\tilde{\varphi}),
$$

is an epimorphic image of the abelianization of $U$, namely the quotient of $U / U^{\prime}$ by the kernel of $\tilde{\varphi}$, which is given by

$$
\operatorname{ker}(\tilde{\varphi})=\left(U^{\prime} \cdot \operatorname{ker}(\varphi) \cap U\right) / U^{\prime}
$$


3) Moreover, the map $\tilde{\varphi}$ is an isomorphism, and the quotients $V / V^{\prime} \simeq U / U^{\prime}$ are isomorphic, if and only if

$$
\operatorname{ker}(\varphi) \leq U^{\prime} .
$$

See Figure 2 for a visualization of this situation.

Proof. The statements can be seen in the following manner. The image of the commutator subgroup is given by

$$
\varphi\left(U^{\prime}\right)=\varphi([U, U])=\varphi(\langle[u, v] \mid u, v \in U\rangle)=\langle[\varphi(u), \varphi(v)] \mid u, v \in U\rangle=[\varphi(U), \varphi(U)]=\varphi(U)^{\prime}=V^{\prime} .
$$

The homomorphism $\varphi$ can be restricted to an epimorphism $\left.\varphi\right|_{U}: U \rightarrow \varphi(U)=V$. According to Theorem 7.1, in particular, by the Formulas (7.5) and (7.4) in the appendix, the condition $\varphi\left(U^{\prime}\right)=V^{\prime}$ implies the existence of a uniquely determined epimorphism $\tilde{\varphi}: U / U^{\prime} \rightarrow V / V^{\prime}$ such that $\tilde{\varphi} \circ \omega_{U^{\prime}}=\left.\omega_{V^{\prime}} \circ \varphi\right|_{U}$. The Isomorphism Theorem in Formula (7.7) in the appendix shows that $V / V^{\prime} \simeq\left(U / U^{\prime}\right) / \operatorname{ker}(\tilde{\varphi})$. Furthermore, by the Formulas (7.4) and (7.1), the kernel of $\tilde{\varphi}$ is given explicitly by

$$
\operatorname{ker}(\tilde{\varphi})=\left(\varphi^{-1}\left(\varphi\left(U^{\prime}\right)\right) \cap U\right) / U^{\prime}=\left(U^{\prime} \cdot \operatorname{ker}(\varphi) \cap U\right) / U^{\prime} .
$$

Thus, $\tilde{\varphi}$ is an isomorphism if and only if $\operatorname{ker}(\varphi) \unlhd U^{\prime}(\unlhd U)$.

$\mathbf{P}$ 5.2. Functor of derived quotients. In analogy to section $\S 7.6$ in the appendix, a covariant functor $F: \varphi \mapsto F(\varphi)=\tilde{\varphi}$ can be used to map a morphism $\varphi$ of one category to an induced morphism $\tilde{\varphi}$ of another category.

In the present situation, we denote by $\mathcal{G}$ the category of groups and we define the domain of the functor $F$ as the following category $\mathcal{G}_{s}$. The objects of the category are pairs $(G, U)$ consisting of a group $G$ and a subgroup $U \leq G$,

$$
\operatorname{Obj}\left(\mathcal{G}_{s}\right)=\{(G, U) \mid G \in \operatorname{Obj}(\mathcal{G}), U \leq G\} .
$$

For two objects $(G, U),(H, V) \in \operatorname{Obj}\left(\mathcal{G}_{s}\right)$, the set of morphisms $\operatorname{Mor}_{\mathcal{G}_{s}}((G, U),(H, V))$ consists of epimorphisms $\varphi: G \rightarrow H$ such that $\varphi(G)=H$ and $\varphi(U)=V$, briefly written as arrows $\varphi:(G, U) \rightarrow(H, V)$,

$$
\operatorname{Mor}_{\mathcal{G}_{\mathrm{s}}}((G, U),(H, V))=\left\{\varphi \in \operatorname{Mor}_{\mathcal{G}}(G, H) \mid \varphi(G)=H, \varphi(U)=V\right\} .
$$

The functor $F: \mathcal{G}_{s} \rightarrow \mathcal{A}$ from this category $\mathcal{G}_{s}$ to the category $\mathcal{A}$ of abelian groups maps a pair $(G, U) \in \operatorname{Obj}\left(\mathcal{G}_{s}\right)$ to the commutator quotient group $F((G, U)):=U / U^{\prime} \in \operatorname{Obj}(\mathcal{A})$ of the subgroup $U$, and it maps a morphism $\varphi \in \operatorname{Mor}_{\mathcal{G}_{s}}((G, U),(H, V))$ to the induced epimorphism $F(\varphi):=\tilde{\varphi} \in \operatorname{Mor}_{\mathcal{A}}\left(U / U^{\prime}, V / V^{\prime}\right)$ of the restriction $\left.\varphi\right|_{U}: U \rightarrow V$,

$$
F: \mathcal{G}_{s} \rightarrow \mathcal{A}, F((G, U)):=U / U^{\prime}, F(\varphi):=\tilde{\varphi} .
$$

Existence and uniqueness of $F(\varphi):=\tilde{\varphi}$ have been proved in Theorem 5.1 under the assumption that $\varphi(U)=V$, which is satisfied according to the definition of the arrow $\varphi:(G, U) \rightarrow(H, V)$ and automatically implies $\varphi\left(U^{\prime}\right)=V^{\prime}$.

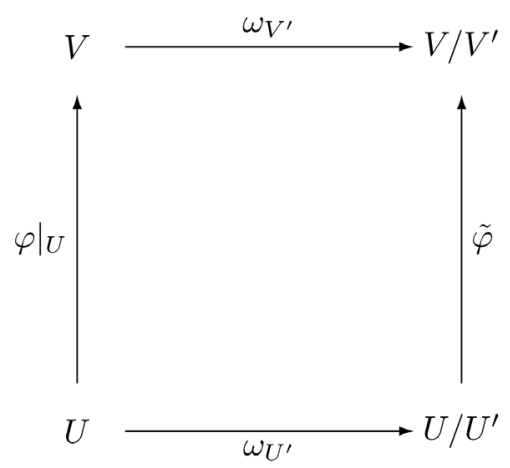

Figure 2. Induced homomorphism of derived quotients. 
Definition 5.1. Due to the results in Theorem 5.1, it makes sense to define a pre-order of transfer targets on the image $F\left(\operatorname{Obj}\left(\mathcal{G}_{s}\right)\right)$ of the functor $F$ in the object class $\operatorname{Obj}(\mathcal{A})$ of the category $\mathcal{A}$ of abelian groups in the following manner.

For two objects $(G, U),(H, V) \in \operatorname{Obj}\left(\mathcal{G}_{s}\right)$, a morphism $\varphi \in \operatorname{Mor}_{\mathcal{G}_{s}}((G, U),(H, V))$, and the images $F((G, U))=U / U^{\prime}, F((H, V))=V / V^{\prime} \in \operatorname{Obj}(\mathcal{A})$, and $F(\varphi)=\tilde{\varphi} \in \operatorname{Mor}_{\mathcal{A}}\left(U / U^{\prime}, V / V^{\prime}\right)$, let (non-strict) precedence be defined by

$$
V / V^{\prime} \preceq U / U^{\prime}: \Leftrightarrow V / V^{\prime} \simeq\left(U / U^{\prime}\right) / \operatorname{ker}(\tilde{\varphi}),
$$

and let equality be defined by

$$
V / V^{\prime}=U / U^{\prime}: \Leftrightarrow V / V^{\prime} \simeq U / U^{\prime},
$$

if the induced epimorphism $\tilde{\varphi}: U / U^{\prime} \rightarrow V / V^{\prime}$ is an isomorphism.

Corollary 5.1. If both components of the pairs $(G, U),(H, V) \in \operatorname{Obj}\left(\mathcal{G}_{s}\right)$ are restricted to Hopfian groups, then the pre-order of transfer targets $V / V^{\prime} \preceq U / U^{\prime}$ is actually a partial order.

Proof. We use the functorial properties of the functor $F$. The reflexivity of the partial order follows from the functorial identity in Formula (7.14), and the transitivity is a consequence of the functorial compositum in Formula (7.15), given in the appendix. The antisymmetry might be a problem for infinite groups, since it is known that there exist so-called non-Hopfian groups. However, for finite groups, and more generally for Hopfian groups, it is due to the implication $V / V^{\prime} \simeq\left(U / U^{\prime}\right) / \operatorname{ker}\left(\tilde{\varphi}_{1}\right) \simeq\left(\left(V / V^{\prime}\right) / \operatorname{ker}\left(\tilde{\varphi}_{2}\right)\right) / \operatorname{ker}\left(\tilde{\varphi}_{1}\right)$ $\Rightarrow \operatorname{ker}\left(\tilde{\varphi}_{1}\right)=\operatorname{ker}\left(\tilde{\varphi}_{2}\right)=1$.

\subsection{Singulets of Transfer Kernels}

Suppose that $G$ and $T$ are groups, $H=\operatorname{im}(\varphi)=\varphi(G) \leq T$ is the image of $G$ under a homomorphism $\varphi: G \rightarrow T$, and $V=\varphi(U)$ is the image of a subgroup $U \leq G$ of finite index $1 \leq n:=(G: U)<\infty$. Let $T_{G, U}$ be the Artin transfer from $G$ to $U / U^{\prime}$.

Theorem 5.2. If $\operatorname{ker}(\varphi) \leq U$, then the image $\left(\varphi\left(\ell_{1}\right), \cdots, \varphi\left(\ell_{n}\right)\right)$ of a left transversal $\left(\ell_{1}, \cdots, \ell_{n}\right)$ of $U$ in $G$ is a left transversal of $V$ in $H$, the index $(H: V)=(G: U)=n<\infty$ remains the same and is therefore finite, and the Artin transfer $T_{H, V}$ from $H$ to $V / V^{\prime}$ exists.

1) The following connections exist between the two Artin transfers: the required condition for the composita of mappings in the commutative diagram in Figure 3,

$$
\tilde{\varphi} \circ T_{G, U}=T_{H, V} \circ \varphi,
$$

and, consequently, the inclusion of the kernels,

$$
\varphi\left(\operatorname{ker}\left(T_{G, U}\right)\right) \leq \operatorname{ker}\left(T_{H, V}\right) .
$$

2) A sufficient (but not necessary) condition for the equality of the kernels is given by

$$
\operatorname{ker}\left(T_{H, V}\right)=\varphi\left(\operatorname{ker}\left(T_{G, U}\right)\right) \text { if } \operatorname{ker}(\varphi) \leq U^{\prime} .
$$

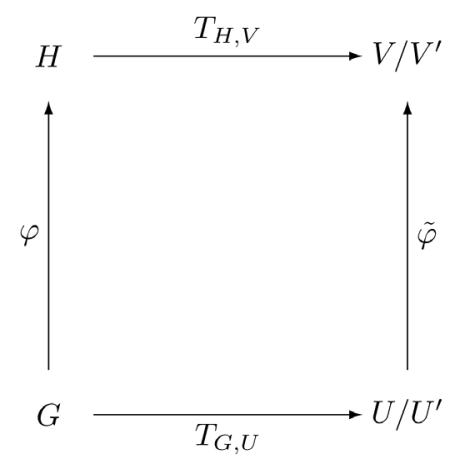

Figure 3. Epimorphism and Artin transfer. 
See Figure 3 for a visualization of this scenario.

Proof. The truth of these statements can be justified in the following way. The first part has been proved in Proposition 2.1 already: Let $\left(\ell_{1}, \cdots, \ell_{n}\right)$ be a left transversal of $U$ in $G$. Then $G=\bigcup_{i=1}^{n} \ell_{i} U$ is a disjoint union but the union $\varphi(G)=\bigcup_{i=1}^{n} \varphi\left(\ell_{i}\right) \varphi(U)$ is not necessarily disjoint. For $1 \leq j, k \leq n$, we have $\varphi\left(\ell_{j}\right) \varphi(U)=\varphi\left(\ell_{k}\right) \varphi(U) \Leftrightarrow \varphi(U)=\varphi\left(\ell_{j}\right)^{-1} \varphi\left(\ell_{k}\right) \varphi(U)=\varphi\left(\ell_{j}^{-1} \ell_{k}\right) \varphi(U) \Leftrightarrow \varphi\left(\ell_{j}^{-1} \ell_{k}\right)=\varphi(u)$ for some element $u \in U \Leftrightarrow \varphi\left(u^{-1} \ell_{j}^{-1} \ell_{k}\right)=1 \Leftrightarrow u^{-1} \ell_{j}^{-1} \ell_{k}=: x \in \operatorname{ker}(\varphi)$. However, if the condition $\operatorname{ker}(\varphi) \unlhd U$ is satisfied, then we are able to conclude that

$\ell_{j}^{-1} \ell_{k}=u x \in U$, and thus $j=k$.

Let $\tilde{\varphi}: U / U^{\prime} \rightarrow V / V^{\prime}$ be the epimorphism obtained in the manner indicated in the proof of Theorem 5.1 and Formula (5.2). For the image of $x \in G$ under the Artin transfer, we obtain

$$
\begin{aligned}
\tilde{\varphi}\left(T_{G, U}(x)\right) & =\tilde{\varphi}\left(\prod_{i=1}^{n} \ell_{\lambda_{x}(i)}^{-1} x \ell_{i} \cdot U^{\prime}\right)=\prod_{i=1}^{n} \tilde{\varphi}\left(\ell_{\lambda_{x}(i)}^{-1} x \ell_{i} \cdot U^{\prime}\right) \\
& =\prod_{i=1}^{n} \varphi\left(\ell_{\lambda_{x}(i)}^{-1} x \ell_{i}\right) \cdot \varphi\left(U^{\prime}\right)=\prod_{i=1}^{n} \varphi\left(\ell_{\lambda_{x}(i)}\right)^{-1} \varphi(x) \varphi\left(\ell_{i}\right) \cdot \varphi\left(U^{\prime}\right) .
\end{aligned}
$$

Since $\varphi\left(U^{\prime}\right)=\varphi(U)^{\prime}=V^{\prime}$, the right hand side equals $T_{H, V}(\varphi(x))$, provided that $\left(\varphi\left(\ell_{1}\right), \cdots, \varphi\left(\ell_{n}\right)\right)$ is a left transversal of $V$ in $H$, which is correct when $\operatorname{ker}(\varphi) \unlhd U$. This shows that the diagram in Figure 3 is commutative, that is, $\tilde{\varphi} \circ T_{G, U}=T_{H, V} \circ \varphi$. It also yields the connection between the permutations $\lambda_{\varphi(x)}=\lambda_{x}$ and the monomials $u_{\varphi(x)}(i)=\varphi\left(u_{x}(i)\right)$ for all $1 \leq i \leq n$. As a consequence, we obtain the inclusion $\varphi\left(\operatorname{ker}\left(T_{G, U}\right)\right) \leq \operatorname{ker}\left(T_{H, V}\right)$, if $\operatorname{ker}(\varphi) \unlhd U$. Finally, if $\operatorname{ker}(\varphi) \unlhd U^{\prime}$, then the previous section has shown that $\tilde{\varphi}$ is an isomorphism. Using the inverse isomorphism, we get $T_{G, U}=\tilde{\varphi}^{-1} \circ T_{H, V} \circ \varphi$, which proves the equation $\varphi\left(\operatorname{ker}\left(T_{G, U}\right)\right)=\operatorname{ker}\left(T_{H, V}\right)$. More explicitly, we have the following chain of equivalences and implications:

$$
x \in \operatorname{ker}\left(T_{G, U}\right) \Leftrightarrow T_{G, U}(x)=1 \Rightarrow 1=\tilde{\varphi}\left(T_{G, U}(x)\right)=T_{H, V}(\varphi(x)) \Leftrightarrow \varphi(x) \in \operatorname{ker}\left(T_{H, V}\right) .
$$

Conversely, $\varphi(x) \in \operatorname{ker}\left(T_{H, V}\right)$ only implies $T_{G, U}(x) \in \operatorname{ker}(\tilde{\varphi})=\left(U^{\prime} \cdot \operatorname{ker}(\varphi) \cap U\right) / U^{\prime}$. Therefore, we certainly have $\operatorname{ker}\left(T_{H, V}\right)=\varphi\left(\operatorname{ker}\left(T_{G, U}\right)\right)$ if $\operatorname{ker}(\varphi) \leq U^{\prime}$, which is, however, not necessary.

$P$ 5.3. Artin transfers as natural transformations. Artin transfers $T_{G, U}$ can be viewed as components of a natural transformation $\mathrm{T}$ between two functors ? and F from the following category $\mathcal{G}_{f}$ to the usual category $\mathcal{G}$ of groups.

The objects of the category $\mathcal{G}_{f}$ are pairs $(G, U)$ consisting of a group $G$ and a subgroup $U \leq G$ of finite index $(G: U)<\infty$,

$$
\operatorname{Obj}\left(\mathcal{G}_{f}\right)=\{(G, U) \mid G \in \operatorname{Obj}(\mathcal{G}), U \leq G,(G: U)<\infty\} .
$$

For two objects $(G, U),(H, V) \in \operatorname{Obj}\left(\mathcal{G}_{f}\right)$, the set of morphisms $\operatorname{Mor}_{\mathcal{G}_{f}}((G, U),(H, V))$ consists of epimorphisms $\varphi: G \rightarrow H$ satisfying $\varphi(G)=H, \varphi(U)=V$, and the additional condition $\operatorname{ker}(\varphi) \leq U$ for their kernels, briefly written as arrows $\varphi:(G, U) \rightarrow(H, V)$,

$$
\operatorname{Mor}_{\mathcal{G}_{f}}((G, U),(H, V))=\left\{\varphi \in \operatorname{Mor}_{\mathcal{G}}(G, H) \mid \varphi(G)=H, \varphi(U)=V, \operatorname{ker}(\varphi) \leq U\right\} .
$$

The forgetful functor ?: $\mathcal{G}_{f} \rightarrow \mathcal{G}$ from this category $\mathcal{G}_{f}$ to the category $\mathcal{G}$ of groups maps a pair. $(G, U) \in \operatorname{Obj}\left(\mathcal{G}_{f}\right)$ to its first component $?((G, U)):=G \in \operatorname{Obj}(\mathcal{G})$, and it maps a morphism $\varphi \in \operatorname{Mor}_{\mathcal{G}_{f}}((G, U),(H, V))$ to the underlying epimorphism $?(\varphi):=\varphi \in \operatorname{Mor}_{\mathcal{G}}(G, H)$.

$$
?: \mathcal{G}_{f} \rightarrow \mathcal{G}, ?((G, U)):=G, ?(\varphi):=\varphi \text {. }
$$

The functor $F: \mathcal{G}_{f} \rightarrow \mathcal{G}$ from $\mathcal{G}_{f}$ to the category $F: \mathcal{G}_{f} \rightarrow \mathcal{G}$ of groups maps a pair $(G, U) \in \operatorname{Obj}\left(\mathcal{G}_{f}\right)$ to the commutator quotient group $F((G, U)):=U / U^{\prime} \in \operatorname{Obj}(\mathcal{G})$ of the subgroup $U$, and it maps a morphism $\varphi \in \operatorname{Mor}_{\mathcal{G}_{f}}((G, U),(H, V))$ to the induced epimorphism $F(\varphi):=\tilde{\varphi} \in \operatorname{Mor}_{\mathcal{G}}\left(U / U^{\prime}, V / V^{\prime}\right)$ of the restriction $\left.\varphi\right|_{U}: U \rightarrow V$. Note that we must abstain here from letting $F$ map into the subcategory $\mathcal{A}$ of abelian groups. 


$$
F: \mathcal{G}_{f} \rightarrow \mathcal{G}, F((G, U)):=U / U^{\prime}, F(\varphi):=\tilde{\varphi}
$$

The system $T$ of all Artin transfers fulfils the requirements for a natural transformation $T:$ ? $\rightarrow F$ between these two functors, since we have

$$
F(\varphi) \circ T_{G, U}=\tilde{\varphi} \circ T_{G, U}=T_{H, V} \circ \varphi=T_{H, V} \circ ?(\varphi),
$$

for every morphism $\varphi:(G, U) \rightarrow(H, V)$ of the category $\mathcal{G}_{f}$.

Definition 5.2. Due to the results in Theorem 5.2, it makes sense to define a pre-order of transfer kernels on the kernels $\operatorname{ker}\left(T_{G, U}\right)$ of the components $T_{G, U}$ of the natural transformation $T$ in the object class $\operatorname{Obj}(\mathcal{G})$ of the category $\mathcal{G}$ of groups in the following manner.

For two objects $(G, U),(H, V) \in \operatorname{Obj}\left(\mathcal{G}_{f}\right)$, a morphism $\varphi \in \operatorname{Mor}_{\mathcal{G}_{f}}((G, U),(H, V))$, and the images $F((G, U))=U / U^{\prime}, F((H, V))=V / V^{\prime} \in \operatorname{Obj}(\mathcal{G})$, and $F(\varphi)=\tilde{\varphi} \in \operatorname{Mor}_{\mathcal{G}}\left(U / U^{\prime}, V / V^{\prime}\right)$,

let (non-strict) precedence be defined by

$$
\operatorname{ker}\left(T_{G, U}\right) \preceq \operatorname{ker}\left(T_{H, V}\right): \Leftrightarrow \varphi\left(\operatorname{ker}\left(T_{G, U}\right)\right) \leq \operatorname{ker}\left(T_{H, V}\right),
$$

and let equality be defined by

$$
\operatorname{ker}\left(T_{G, U}\right)=\operatorname{ker}\left(T_{H, V}\right): \Leftrightarrow \varphi\left(\operatorname{ker}\left(T_{G, U}\right)\right)=\operatorname{ker}\left(T_{H, V}\right),
$$

if the induced epimorphism $\tilde{\varphi}: U / U^{\prime} \rightarrow V / V^{\prime}$ is an isomorphism.

Corollary 5.2. If both components of the pairs $(G, U),(H, V) \in \operatorname{Obj}\left(\mathcal{G}_{s}\right)$ are restricted to Hopfian groups, then the pre-order of transfer kernels $\operatorname{ker}\left(T_{G, U}\right) \preceq \operatorname{ker}\left(T_{H, V}\right)$ is actually a partial order.

Proof. Similarly as in the proof of Corollary 5.1, we use the properties of the functor $F$. The reflexivity is due to the functorial identity in Formula (7.14). The transitivity is due to the functorial compositum in Formula (7.15), where we have to observe the relations $\operatorname{ker}(\varphi) \leq U, \operatorname{ker}(\psi) \leq V$, and Formula (7.1) in the appendix for verifying the kernel relation

$$
\operatorname{ker}(\psi \circ \varphi)=(\psi \circ \varphi)^{-1}(1)=\varphi^{-1}\left(\psi^{-1}(1)\right)=\varphi^{-1}(\operatorname{ker}(\psi)) \leq \varphi^{-1}(V)=\varphi^{-1}(\varphi(U))=U \cdot \operatorname{ker}(\varphi)=U
$$

additionally to the image relation

$$
(\psi \circ \varphi)(U)=\psi(\varphi(U))=\psi(V)=W .
$$

The antisymmetry is certainly satisfied for finite groups, and more generally for Hopfian groups.

\subsection{Multiplets of Transfer Targets and Kernels}

Instead of viewing various pairs $(G, U)$ which share the same first component $G$ as distinct objects in the categories $\mathcal{G}_{s}$, resp. $\mathcal{G}_{f}$, which we used for describing singulets of transfer targets, resp. kernels, we now consider a collective accumulation of singulets in multiplets. For this purpose, we shall define a new category $\mathcal{G}_{(f)}$ of families, which generalizes the category $\mathcal{G}_{f}$, rather than the category $\mathcal{G}_{s}$. However, we have to prepare this definition with a criterion for the compatibility of a system of subgroups with its image under a homomorphism.

Proposition 5.1. See also ([7], Thm.2.3.4, p. 29); ([8], Satz 3.10, p. 16); ([9], Thm.2.4, p. 6); ([13], Thm.X.21, p. 340).

For an epimorphism $\phi: G \rightarrow H$ of groups, the associated set mappings

$$
\phi: \mathcal{U} \rightarrow \mathcal{V} \text { and } \phi^{-1}: \mathcal{V} \rightarrow \mathcal{U}
$$

are inverse bijections between the following systems of subgroups

$$
\mathcal{U}:=\{U \mid \operatorname{ker}(\phi) \leq U \leq G\} \text { and } \mathcal{V}:=\{V \mid V \leq H\}
$$

Proof. The fourth and fifth statement of Lemma 7.1 in the appendix show that usually the associated set mappings $\phi^{-1}$ and $\phi$ of a homomorphism $\phi: G \rightarrow H$ are not inverse bijections between systems of subgroups of $G$ and $H$. However, if we replace the homomorphism $\phi$ by an epimorphism with $H=\phi(G)$, then 
the Formula (7.2) yields the first desired equality

$$
\phi\left(\phi^{-1}(V)\right)=\phi(G) \cap V=H \cap V=V \text { for all } V \in \mathcal{V}:=\{V \mid V \leq H\} .
$$

Guided by the property $\operatorname{ker}(\phi)=\phi^{-1}(1) \leq \phi^{-1}(V)$ of all pre-images $\phi^{-1}(V)$ of $V \in \mathcal{V}$, we define a restricted system of subgroups of the domain $G$,

$$
\mathcal{U}:=\{U \mid \operatorname{ker}(\phi) \leq U \leq G\},
$$

and, according to Formula (7.1.), we consequently obtain the second required equality

$$
\phi^{-1}(\phi(U))=U \cdot \operatorname{ker}(\phi)=U \text { for all } U \in \mathcal{U},
$$

which yields the crucial pair of inverse set bijections

$$
\phi^{-1}: \mathcal{V} \rightarrow \mathcal{U} \text { and } \phi: \mathcal{U} \rightarrow \mathcal{V}
$$

P 5.4. After this preparation, we are able to specify the new category $\mathcal{G}_{(f)}$. The objects of the category $\mathcal{G}_{(f)}$ are pairs $\left(G,\left(U_{i}\right)_{i \in I}\right)$ consisting of a group $G$ and the family of all subgroups $U_{i} \leq G$ with finite index $\left(G: U_{i}\right)<\infty$,

$$
\operatorname{Obj}\left(\mathcal{G}_{(f)}\right)=\left\{\left(G,\left(U_{i}\right)_{i \in I}\right) \mid G \in \operatorname{Obj}(\mathcal{G}), U_{i} \leq G,\left(G: U_{i}\right)<\infty \text {, for all } i \in I\right\},
$$

where $I$ denotes a suitable indexing set. Note that $G$ itself is one of the subgroups $U_{i}$.

The morphisms of the new category are subject to more restrictive conditions, which concern entire families of subgroups instead of just a single subgroup.

For two objects $\left(G,\left(U_{i}\right)_{i \in I}\right),\left(H,\left(V_{i}\right)_{i \in I}\right) \in \operatorname{Obj}\left(\mathcal{G}_{(f)}\right)$, the set $\operatorname{Mor}_{\mathcal{G}_{(f)}}\left(\left(G,\left(U_{i}\right)_{i \in I}\right),\left(H,\left(V_{i}\right)_{i \in I}\right)\right)$ of morphisms consists of epimorphisms $\varphi: G \rightarrow H$ satisfying $\varphi(G)=H$, the image conditions $\varphi\left(U_{i}\right)=V_{i}$, and the kernel conditions $\operatorname{ker}(\varphi) \leq U_{i}$, which imply the pre-image conditions $\varphi^{-1}\left(V_{i}\right)=U_{i}$, for all $i \in I$, briefly written as arrows $\varphi:\left(G,\left(U_{i}\right)_{i \in I}\right) \rightarrow\left(H,\left(V_{i}\right)_{i \in I}\right)$,

$$
\operatorname{Mor}_{\mathcal{G}_{(f)}}\left(\left(G,\left(U_{i}\right)_{i \in I}\right),\left(H,\left(V_{i}\right)_{i \in I}\right)\right)=\left\{\varphi \in \operatorname{Mor}_{\mathcal{G}}(G, H) \mid \varphi\left(U_{i}\right)=V_{i}, \operatorname{ker}(\varphi) \leq U_{i} \text {, for all } i \in I\right\} \text {. }
$$

Note that, in view of Proposition 5.1, we can always use the same indexing set $I$ for the domain and for the codomain of morphisms, provided they satisfy the required kernel condition.

Now we come to the essential definition of Artin transfer patterns.

Definition 5.3. Let $\left(G,\left(U_{i}\right)_{i \in I}\right) \in \operatorname{Obj}\left(\mathcal{G}_{(f)}\right)$ be an object of the category $\mathcal{G}_{(f)}$.

The transfer target type (TTT) of $G$ is the family

$$
\tau(G):=\left(U_{i} / U_{i}^{\prime}\right)_{i \in I} \text { of abelian groups in } \operatorname{Obj}(\mathcal{A}) .
$$

The transfer kernel type (TKT) of $G$ is the family

$$
\varkappa(G):=\left(\operatorname{ker}\left(T_{G, U_{i}}\right)\right)_{i \in I} \text { of groups in } \operatorname{Obj}(\mathcal{G}) .
$$

The complete Artin pattern of $G$ is the pair

$$
\operatorname{AP}_{c}(G):=(\tau(G), \varkappa(G)) .
$$

P 5.5. The natural partial order on TTTs and TKTs is reduced to the partial order on the components, according to the Definitions 5.1 and 5.2 .

Definition 5.4. Let $\left(G,\left(U_{i}\right)_{i \in I}\right),\left(H,\left(V_{i}\right)_{i \in I}\right) \in \operatorname{Obj}\left(\mathcal{G}_{(f)}\right)$ be two objects of the category $\mathcal{G}_{(f)}$, where all members of the families $\left(U_{i}\right)_{i \in I}$ and $\left(V_{i}\right)_{i \in I}$ are Hopfian groups.

Then (non-strict) precedence of TTTs is defined by 


$$
\tau(H) \preceq \tau(G): \Leftrightarrow V_{i} / V_{i}^{\prime} \preceq U_{i} / U_{i}^{\prime}, \text { for all } i \in I,
$$

and equality of TTTs is defined by

$$
\tau(H)=\tau(G): \Leftrightarrow V_{i} / V_{i}^{\prime}=U_{i} / U_{i}^{\prime}, \text { for all } i \in I .
$$

(Non-strict) precedence of TKTs is defined by

$$
\varkappa(G) \preceq \varkappa(H): \Leftrightarrow \operatorname{ker}\left(T_{G, U_{i}}\right) \preceq \operatorname{ker}\left(T_{H, V_{i}}\right) \text {, for all } i \in I,
$$

and equality of TKTs is defined by

$$
\varkappa(G)=\varkappa(H): \Leftrightarrow \operatorname{ker}\left(T_{G, U_{i}}\right)=\operatorname{ker}\left(T_{H, V_{i}}\right) \text {, for all } i \in I .
$$

We partition the indexing set $I$ in two disjoint components, according to whether components of the Artin pattern remain fixed or change under an epimorphism.

Definition 5.5. Let $\left(G,\left(U_{i}\right)_{i \in I}\right),\left(H,\left(V_{i}\right)_{i \in I}\right) \in \operatorname{Obj}\left(\mathcal{G}_{(f)}\right)$ be two objects of the category $\mathcal{G}_{(f)}$, and let $\varphi \in \operatorname{Mor}_{\mathcal{G}_{(f)}}\left(\left(G,\left(U_{i}\right)_{i \in I}\right),\left(H,\left(V_{i}\right)_{i \in I}\right)\right)$ be a morphism between these objects.

The stable part and the polarized part of the Artin pattern $\operatorname{AP}(G)$ of $G$ with respect to $\varphi$ are defined by

$$
\begin{aligned}
& \operatorname{Stb}_{\varphi}(G):=\left\{i \in I \mid \operatorname{ker}(\varphi) \leq U_{i}^{\prime}\right\}, \\
& \operatorname{Pol}_{\varphi}(G):=\left\{i \in I \mid \operatorname{ker}(\varphi) \nless U_{i}^{\prime}\right\} .
\end{aligned}
$$

Accordingly, we have

$$
\begin{aligned}
& V_{i} / V_{i}^{\prime}=U_{i} / U_{i}^{\prime} \text { and } \operatorname{ker}\left(T_{G, U_{i}}\right)=\operatorname{ker}\left(T_{H, V_{i}}\right) \text {, for all } i \in \operatorname{Stb}_{\varphi}(G), \\
& V_{i} / V_{i}^{\prime} \prec U_{i} / U_{i}^{\prime} \text { and } \operatorname{ker}\left(T_{G, U_{i}}\right) \preceq \operatorname{ker}\left(T_{H, V_{i}}\right), \text { for all } i \in \operatorname{Pol}_{\varphi}(G) .
\end{aligned}
$$

Note that the precedence of polarized targets is strict as opposed to polarized kernels.

\subsection{The Artin Pattern on a Descendant Tree}

P 5.6. Before we specialize to the usual kinds of descendant trees of finite p-groups ([3], §4, pp. 163-164) we consider an abstract form of a rooted directed tree $\mathcal{T}$, which is characterized by two relations.

Firstly, a basic relation $\pi(G)<G$ between parent and child (also called immediate descendant), corresponding to a directed edge $G \rightarrow \pi(G)$ of the tree, for any vertex $G \in \mathcal{T} \backslash\{R\}$ which is different from the root $R$ of the tree.

Secondly, an induced non-strict partial order relation, $\pi^{n}(G) \leq G$ for some integer $n \geq 0$, between ancestor and descendant, corresponding to a path $G=\pi^{0}(G) \rightarrow \pi^{1}(G) \rightarrow \cdots \rightarrow \pi^{n}(G)$ of directed edges, for an arbitrary vertex $G \in \mathcal{T}$, that is, the ancestor $\pi^{n}(G)$ is an iterated parent of the descendant. Note that only an empty path with $n=0$ starts from the root $R$ of the tree, which has no parent.

Just a brief justification of the partial order: Reflexivity is due to the relation $G=\pi^{0}(G)$. Transitivity follows from the rule $\pi^{m}\left(\pi^{n}(G)\right)=\pi^{m+n}(G)$. Antisymmetry is a consequence of the absence of cycles, that is, $H=\pi^{m}(G)=\pi^{m}\left(\pi^{n}(H)\right)=\pi^{m+n}(H)$ implies $m=n=0$ and thus $H=G$.

P 5.7. The category of a tree. Now let $\mathcal{T}$ be a rooted directed tree whose vertices are groups $G \in \operatorname{Obj}(\mathcal{G})$. Then we define $\mathcal{G}_{\mathcal{T}}$, the category associated with $\mathcal{T}$, as a subcategory of the category $\mathcal{G}_{(f)}$ which was introduced in the Formulas (5.23) and (5.24).

The objects of the category $\mathcal{G}_{\mathcal{T}}$ are those pairs $\left(G,\left(U_{i}\right)_{i \in I}\right)$ in the object class of the category $\mathcal{G}_{(f)}$ whose first component is a vertex of the tree $\mathcal{T}$,

$$
\operatorname{Obj}\left(\mathcal{G}_{\mathcal{T}}\right)=\left\{\left(G,\left(U_{i}\right)_{i \in I}\right) \in \operatorname{Obj}\left(\mathcal{G}_{(f)}\right) \mid G \in \mathcal{T}\right\} .
$$

The morphisms of the category $\mathcal{G}_{\mathcal{T}}$ are selected along the paths of the tree $\mathcal{T}$ only. 
For two objects $\left(G,\left(U_{i}\right)_{i \in I}\right),\left(H,\left(V_{i}\right)_{i \in I}\right) \in \operatorname{Obj}\left(\mathcal{G}_{\mathcal{T}}\right)$, the set $\operatorname{Mor}_{\mathcal{G}_{\mathcal{T}}}\left(\left(G,\left(U_{i}\right)_{i \in I}\right),\left(H,\left(V_{i}\right)_{i \in I}\right)\right)$ of morphisms is either empty or consists of a single element only,

$$
\operatorname{Mor}_{\mathcal{G}_{\mathcal{T}}}\left(\left(G,\left(U_{i}\right)_{i \in I}\right),\left(H,\left(V_{i}\right)_{i \in I}\right)\right)= \begin{cases}\varnothing & \text { if } H \text { is not an ancestor of } G, \\ \left\{\pi^{n}\right\} & \text { if } H=\pi^{n}(G) \text { for some integer } n \geq 0 .\end{cases}
$$

In the case of an ancestor-descendant relation between $H$ and $G$, the specification of the supercategory $\mathcal{G}_{(f)}$ enforces the following constraints on the unique morphism $\pi^{n}$ : the image relations $\pi^{n}\left(U_{i}\right)=V_{i}$ and the kernel relations $\operatorname{ker}\left(\pi^{n}\right) \leq U_{i}$, for all $i \in I$.

$\mathbf{P}$ 5.8. At this position, we must start to be more concrete. In the descendant tree $\mathcal{T}=\mathcal{T}(R)$ of a group $\mathrm{R}$, which is the root of the tree, the formal parent operator $\pi$ gets a second meaning as a natural projection $\pi: G \rightarrow \pi(G)=G / N, x \mapsto \pi(x)=x N$, from the child $G$ onto its parent $\pi(G)$, which is always the quotient of $G$ by a suitable normal subgroup $N \unlhd G$. To be precise, the epimorphism $\pi=\pi_{G}$ with kernel $\operatorname{ker}(\pi)=N$ is actually dependent on its domain G. Therefore, the formal power $\pi^{n}$ is only a convenient abbreviation for the compositum $\pi_{\pi^{n-1}(G)} \circ \cdots \circ \pi_{\pi^{2}(G)} \circ \pi_{\pi(G)} \circ \pi_{G}$.

As described in [3], there are several possible selections of the normal subgroup $N$ in the parent definition $G / N$. Here, we would like to emphasize the following three choices of characteristic subgroups $N$ of the child $G$. If $p$ denotes a prime number and $\mathcal{T}(R)$ is the descendant tree of a finite $p$-group $R$, then it is usual to take for $N \unlhd G$

1) either the last non-trivial member $N:=\gamma_{c}(G)$ of the lower central series of $G$

2) or the last non-trivial member $N:=P_{\tilde{\tilde{c}}-1}(G)$ of the lower exponent- $p$ central series of $G$

3) or the last non-trivial member $N:=G^{(d-1)}$ of the derived series of $G$,

where $c=\operatorname{cl}(G)$ denotes the nilpotency class, $\tilde{c}=\operatorname{cl}_{p}(G)$ the lower exponent $p$-class, and $d=\operatorname{dl}(G)$ the derived length of $G$, respectively.

Note that every descendant tree of finite $p$-groups is subtree of a descendant tree with abelian root. Therefore, it is no loss of generality to restrict our attention to descendant trees with abelian roots.

Theorem 5.3. A uniform warranty for the comparability of the Artin patterns $(\tau(G), \varkappa(G))$ of all vertices $G$ of a descendant tree $\mathcal{T}=\mathcal{T}(R)$ of finite p-groups with abelian root $R$, in the sense of the natural partial order, is given by the following restriction of the family of subgroups $\left(U_{i}\right)_{i \in I}$ in the corresponding object $\left(G,\left(U_{i}\right)_{i \in I}\right)$ of the category $\mathcal{G}_{\mathcal{T}}$. The restriction depends on the definition of a parent $\pi(G)$ in the descendant tree.

1) $\gamma_{2}(G) \leq U_{i}$ for all $i \in I$, when $\pi(G)=G / \gamma_{c}(G)$ with $c=\operatorname{cl}(G)$.

2) $P_{1}(G) \leq U_{i}$ for all $i \in I$, when $\pi(G)=G / P_{\tilde{c}-1}(G)$ with $\tilde{c}=\mathrm{cl}_{p}(G)$.

3) $G^{(1)} \leq U_{i}$ for all $i \in I$, when $\pi(G)=G / G^{(d-1)}$ with $d=\operatorname{dl}(G)$.

Proof. If parents are defined by $\pi(G)=G / \gamma_{c}(G)$ with $c=\operatorname{cl}(G)$, then we have $\operatorname{ker}(\pi)=\gamma_{c}(G)$ and $\operatorname{ker}\left(\pi^{n}\right)=\gamma_{c+1-n}(G)$ for any $0 \leq n<c$. The largest of these kernels arises for $n=c-1$. Therefore, uniform comparability of Artin patterns is warranted by the restriction $\operatorname{ker}\left(\pi^{c-1}\right)=\gamma_{2}(G) \leq U_{i}$ for all $i \in I$.

The parent definition $\pi(G)=G / P_{c-1}(G)$ with $c=\mathrm{cl}_{p}(G)$ implies $\operatorname{ker}(\pi)=P_{c-1}(G)$ and $\operatorname{ker}\left(\pi^{n}\right)=P_{c-n}(G)$ for any $0 \leq n<c$. The largest of these kernels arises for $n=c-1$. Consequently, a uniform comparability of Artin patterns is guaranteed by the restriction $\operatorname{ker}\left(\pi^{c-1}\right)=P_{1}(G) \leq U_{i}$ for all $i \in I$.

Finally, in the case of the parent definition $\pi(G)=G / G^{(d-1)}$ with $d=\operatorname{dl}(G)$, we have $\operatorname{ker}(\pi)=G^{(d-1)}$ and $\operatorname{ker}\left(\pi^{n}\right)=G^{(d-n)}$ for any $0 \leq n<d$. The largest of these kernels arises for $n=d-1$. Consequently, a uniform comparability of Artin patterns is guaranteed by the condition $\operatorname{ker}\left(\pi^{d-1}\right)=G^{(1)} \leq U_{i}$ for all $i \in I$.

P 5.9. Note that the first and third condition coincide since both, $G^{(1)}$ and $\gamma_{2}(G)$, denote the commutator subgroup $G^{\prime}$. So the family $\left(U_{i}\right)_{i \in I}$ is restricted to the normal subgroups which contain $G^{\prime}$, as announced in the paragraph preceding Lemma 3.1.

The second condition restricts the family $\left(U_{i}\right)_{i \in I}$ to the maximal subgroups of $G$ inclusively the group $G$ itself and the Frattini subgroup $P_{1}(G)=\Phi(G)$. 
P 5.10. Since we shall mainly be concerned with the first and third parent definition for descendant trees, that is, either with respect to the lower central series or to the derived series, the comparability condition in Theorem 5.3 suggests the definition of a category $\mathcal{G}_{(h)}$ whose objects are subject to more severe conditions than those in Formula (5.23),

$$
\operatorname{Obj}\left(\mathcal{G}_{(h)}\right)=\left\{\left(G,\left(U_{i}\right)_{i \in I}\right) \mid G \in \operatorname{Obj}(\mathcal{G}),\left(G: G^{\prime}\right)<\infty, G^{\prime} \leq U_{i} \leq G, \text { for all } i \in I\right\},
$$

but whose morphism are defined exactly as in Formula (5.24). The new viewpoint leads to a corresponding modification of Artin transfer patterns.

Definition 5.6. Let $\left(G,\left(U_{i}\right)_{i \in I}\right) \in \operatorname{Obj}\left(\mathcal{G}_{(h)}\right)$ be an object of the category $\mathcal{G}_{(h)}$.

The Artin pattern, more precisely the restricted Artin pattern, of $G$ is the pair

$$
\operatorname{AP}(G):=\operatorname{AP}_{r}(G):=(\tau(G), \varkappa(G)),
$$

whose components, the TTT and the TKT of $G$, are defined as in the Formulas (5.25) and (5.26), but now with respect to the smaller system of subgroups of $G$.

P 5.11. The following Main Theorem shows that any non-metabelian group $G$ with derived length $\operatorname{dl}(G) \geq 3$ and finite abelianization $G / G^{\prime}$ shares its Artin transfer pattern $(\tau(G), \varkappa(G))$, in the restricted sense, with its metabelianization, that is the second derived quotient $G / G^{\prime \prime}$.

Theorem 5.4. (Main Theorem.) Let $G$ be a (non-metabelian) group with finite abelianization $G / G^{\prime}$, and denote by $G^{(n)}, n \geq 0$, the terms of the derived series of $G$, that is $G^{(0)}:=G$ and $G^{(n+1)}:=\left[G^{(n)}, G^{(n)}\right]$ for $n \geq 0$, in particular, $G^{(1)}=G^{\prime}$ and $G^{(2)}=G^{\prime \prime}$, then

1) every subgroup $U \leq G$ which contains the commutator subgroup $G^{\prime}$ is a normal subgroup $U \unlhd G$ of finite index $1 \leq m:=(G: U)<\infty$,

2) for each $G^{\prime} \unlhd U \unlhd G$, there is a chain of normal subgroups

$$
G^{\prime \prime} \unlhd U^{\prime} \unlhd G^{\prime} \unlhd U \unlhd G \text {, and }\left(U / G^{\prime \prime}\right)^{\prime}=U^{\prime} / G^{\prime \prime},
$$

3) for each $G^{\prime} \unlhd U \unlhd G$, the targets of the transfers $T_{G, U}: G \rightarrow U / U^{\prime}$ and

$T_{G / G^{\prime \prime}, U / G^{\prime \prime}}: G / G^{\prime \prime} \rightarrow\left(U / G^{\prime \prime}\right) /\left(U^{\prime} / G^{\prime \prime}\right)$ are equal in the sense of the natural order,

$$
U / U^{\prime}=\left(U / G^{\prime \prime}\right) /\left(U^{\prime} / G^{\prime \prime}\right),
$$

4) for each $G^{\prime} \unlhd U \unlhd G$, the kernels of the transfers $T_{G, U}: G \rightarrow U / U^{\prime}$ and

$T_{G / G^{\prime \prime}, U / G^{\prime \prime}}: G / G^{\prime \prime} \rightarrow\left(U / G^{\prime \prime}\right) /\left(U^{\prime} / G^{\prime \prime}\right)$ are equal in the sense of the natural order,

$$
\operatorname{ker}\left(T_{G, U}\right)=\operatorname{ker}\left(T_{G / G^{\prime \prime}, G^{\prime \prime}}\right) \text {. }
$$

Proof. We use the natural epimorphism $\omega: G \rightarrow G / G^{\prime \prime}, \quad x \mapsto x G^{\prime \prime}$.

1) If $U$ is an intermediate group $G^{\prime} \leq U \leq G$, then $U \unlhd G$ is a normal subgroup of $G$, according to Lemma 3.1. The assumption $\infty>\left(G: G^{\prime}\right)=(G: U) \cdot\left(U: G^{\prime}\right)$ implies that $m:=(G: U)$ is a divisor of the integer $\left(G: G^{\prime}\right)$. Therefore, the Artin transfer $T_{G, U}$ exists.

2) Firstly, $U \leq G$ implies $U^{\prime}=[U, U] \leq[G, G]=G^{\prime}$. Since $U^{\prime}$ is characteristic in $U$, we also have $U^{\prime} \unlhd G^{\prime}$. Similarly, $G^{\prime \prime}$ is characteristic in $G^{\prime}$ and thus normal in $U^{\prime}$. Finally, we obtain

$\left(U / G^{\prime \prime}\right)^{\prime}=\omega(U)^{\prime}=\omega\left(U^{\prime}\right)=U^{\prime} / G^{\prime \prime}$.

3) The mapping $f: U / G^{\prime \prime} \rightarrow U / U^{\prime}, \quad x G^{\prime \prime} \mapsto x U^{\prime}$, is an epimorphism with kernel $\operatorname{ker}(f)=U / G^{\prime \prime}$. Consequently, the isomorphism theorem in Remark 7.3 of the appendix yields the isomorphism $U / U^{\prime}=\operatorname{im}(f) \simeq\left(U / G^{\prime \prime}\right) / \operatorname{ker}(f)=\left(U / G^{\prime \prime}\right) /\left(U^{\prime} / G^{\prime \prime}\right)$.

4) Firstly, the restriction $\left.\omega\right|_{U}: U \rightarrow U / G^{\prime \prime}$ is an epimorphism which induces an isomorphism $\tilde{\omega}: U / U^{\prime} \rightarrow\left(U / G^{\prime \prime}\right) /\left(U^{\prime} / G^{\prime \prime}\right)$, since $\omega\left(U^{\prime}\right)=U / G^{\prime \prime}$ and $\operatorname{ker}(\tilde{\omega})=\omega^{-1}\left(\omega\left(U^{\prime}\right)\right) / U^{\prime}=\left(U^{\prime} \cdot \operatorname{ker}(\omega) \cap U\right) / U^{\prime}=U^{\prime} / U^{\prime} \simeq 1$, according to Theorem 5.1. Secondly, according to Theorem 5.2, the condition $\operatorname{ker}(\omega)=G^{\prime \prime} \leq U$ implies that the index $\left(\left(G / G^{\prime \prime}\right):\left(U / G^{\prime \prime}\right)\right)=(G: U)=m$ is finite, the Artin transfer $T_{G / G^{\prime \prime}, U / G^{\prime \prime}}$ exists, the composite mappings $\tilde{\omega} \circ T_{G, U}=T_{G / G^{\prime \prime}, U / G^{\prime \prime}} \circ \omega$ commute, and, since 
we even have $\operatorname{ker}(\omega)=G^{\prime \prime} \leq U^{\prime}$, the transfer kernels satisfy the relation $\operatorname{ker}\left(T_{G / G^{\prime \prime}}\right)=\omega\left(\operatorname{ker}\left(T_{G, U}\right)\right)=\operatorname{ker}\left(T_{G, U}\right) / G^{\prime \prime}$. In the sense of the natural partial order on transfer kernels this means equality $\operatorname{ker}\left(T_{G / G^{\prime \prime}}\right)=\operatorname{ker}\left(T_{G, U}\right)$, since $G^{\prime} \leq \operatorname{ker}\left(T_{G, U}\right) \leq G$ and thus, similarly as in Proposition 5.1, the map $\omega$ establishes a set bijection between the systems of subgroups $\mathcal{U}:=\left\{U \mid G^{\prime} \leq U \leq G\right\}$ and $\mathcal{V}:=\left\{V \mid G^{\prime} / G^{\prime \prime} \leq V \leq G / G^{\prime \prime}\right\}$, where $G^{\prime} / G^{\prime \prime}=\left(G / G^{\prime \prime}\right)^{\prime}$.

Remark 5.1. At this point it is adequate to emphasize how similar concepts in previous publications are related to the concept of Artin patterns. The restricted Artin pattern $\operatorname{AP}_{r}(G)$ in Definition 5.6 was essentially introduced in ([19], Dfn.1.1, p. 403), for a special case already earlier in ([20], §1, p. 417). The name Artin pattern appears in ([21], Dfn.3.1, p. 747) for the first time. The complete Artin pattern $\operatorname{AP}_{c}(G)$ in Definition 5.3 is new in the present article, but we should point out that it includes the iterated IPADs (index-p abelianization data) in ([18], Dfn.3.5, p. 289) and the iterated IPODs (index-p obstruction data) in ([22], Dfn.4.5).

In a second remark, we emphasize the importance of the preceding Main Theorem for arithmetical applications.

Remark 5.2. In algebraic number theory, Theorem 5.4 has striking consequences for the determination of the length $\ell_{p}(K) \geq 2$ of the p-class tower $\mathrm{F}_{p}^{\infty}(K)$, that is the maximal unramified pro-p extension, of an algebraic number field $K$ with respect to a given prime number $p$. It shows the impossibility of deciding, exclusively with the aid of the restricted Artin pattern $\operatorname{AP}_{r}(G)$, which of several assigned candidates $G$ with distinct derived lengths $\operatorname{dl}(G) \geq 2$ is the actual p-class tower group $\operatorname{Gal}\left(\mathrm{F}_{p}^{\infty}(K) \mid K\right)$. (In contrast, $\ell_{p}(K)=1$ can always be recognized with $\operatorname{AP}_{r}(G)$.)

This is the point where the complete Artin pattern $\operatorname{AP}_{c}(G)$ enters the stage. Most recent investigations by means of iterated IPADs of $2^{\text {nd }}$ order, whose components are contained in $\operatorname{AP}_{c}(G)$, enabled decisions between $2 \leq \ell_{p}(K) \leq 3$ in [18] [22].

Another successful method is to employ cohomological results by I.R. Shafarevich on the relation rank $d_{2}(G)=\operatorname{dim}_{\mathbb{F}_{p}} \mathrm{H}^{2}\left(G, \mathbb{F}_{p}\right)$ for selecting among several candidates $G$ for the $p$-class tower group, in dependence on the torsion-free unit rank of the base field $K$, for instance in [21] [23].

Important examples for the concepts in $\S 5$ are provided in the following subsections.

\subsection{Abelianization of Type $(p, p)$}

Let $G$ be a $p$-group with abelianization $G / G^{\prime}$ of elementary abelian type $(p, p)$. Then $G$ has $p+1$ maximal subgroups $H_{i}<G \quad(1 \leq i \leq p+1)$ of index $\left(G: H_{i}\right)=p$. For each $1 \leq i \leq p+1$, let $T_{i}: G \rightarrow H_{i} / H_{i}^{\prime}$ be the Artin transfer homomorphism from $G$ to the abelianization of $H_{i}$.

Definition 5.7. The family of normal subgroups $\varkappa_{H}(G)=\left(\operatorname{ker}\left(T_{i}\right)\right)_{1 \leq i \leq p+1}$ is called the transfer kernel type (TKT) of $G$ with respect to $H_{1}, \cdots, H_{p+1}$.

Remark 5.3. For brevity, the TKT is identified with the multiplet $(\varkappa(i))_{1 \leq i \leq p+1}$, whose integer components are given by

$$
\varkappa(i)= \begin{cases}0 & \text { if } \operatorname{ker}\left(T_{i}\right)=G, \\ j & \text { if } \operatorname{ker}\left(T_{i}\right)=H_{j} \text { for some } 1 \leq j \leq p+1 .\end{cases}
$$

Here, we take into consideration that each transfer kernel $\operatorname{ker}\left(T_{i}\right)$ must contain the commutator subgroup $G^{\prime}$ of $G$, since the transfer target $H_{i} / H_{i}^{\prime}$ is abelian. However, the minimal case $\operatorname{ker}\left(T_{i}\right)=G^{\prime}$ cannot occur, according to Hilbert's Theorem 94.

A renumeration of the maximal subgroups $K_{i}=H_{\pi(i)}$ and of the transfers $V_{i}=T_{\pi(i)}$ by means of a permutation $\pi \in S_{p+1}$ gives rise to a new TKT $\lambda_{K}(G)=\left(\operatorname{ker}\left(V_{i}\right)\right)_{1 \leq i \leq p+1}$ with respect to $K_{1}, \cdots, K_{p+1}$, identified with $(\lambda(i))_{1 \leq i \leq p+1}$, where 


$$
\lambda(i)= \begin{cases}0 & \text { if } \operatorname{ker}\left(V_{i}\right)=G, \\ j & \text { if } \operatorname{ker}\left(V_{i}\right)=K_{j} \text { for some } 1 \leq j \leq p+1 .\end{cases}
$$

It is adequate to view the TKTs $\lambda_{K}(G) \sim \varkappa_{H}(G)$ as equivalent. Since we have

$$
K_{\lambda(i)}=\operatorname{ker}\left(V_{i}\right)=\operatorname{ker}\left(T_{\pi(i)}\right)=H_{\varkappa(\pi(i))}=K_{\tilde{\pi}^{-1}(\varkappa(\pi(i)))},
$$

the relation between $\lambda$ and $\varkappa$ is given by $\lambda=\tilde{\pi}^{-1} \circ \varkappa \circ \pi$. Therefore, $\lambda$ is another representative of the orbit $\varkappa^{S_{p+1}}$ of $\varkappa$ under the operation $(\pi, \mu) \mapsto \tilde{\pi}^{-1} \circ \mu \circ \pi$ of the symmetric group $S_{p+1}$ on the set of all mappings from $\{1, \cdots, p+1\}$ to $\{0, \cdots, p+1\}$, where the extension $\tilde{\pi} \in S_{p+2}$ of the permutation $\pi \in S_{p+1}$ is defined by $\tilde{\pi}(0)=0$, and we formally put $H_{0}=G, K_{0}=G$.

Definition 5.8. The orbit $\varkappa(G)=\varkappa^{S_{p+1}}$ of any representative $\varkappa$ is an invariant of the p-group $G$ and is called its transfer kernel type, briefly TKT.

Remark 5.4. This definition of $\varkappa(G)$ goes back to the origins of the capitulation theory and was introduced by Scholz and Taussky for $p=3$ in 1934 [24]. Several other authors used this original definition and investigated capitulation problems further. In historical order, Chang in 1977 [25], Chang and Foote in 1980 [26], Heider and Schmithals in 1982 [27], Brink in 1984 [28], Brink and Gold in 1987 [29], Nebelung in 1989 [30], and ourselves in 1991 [31] and in 2012 [32].

In the brief form of the TKT $\varkappa(G)$, the natural order is expressed by $i \prec 0$ for $1 \leq i \leq p+1$.

Let $\# \mathcal{H}_{0}(G):=\#\{1 \leq i \leq p+1 \mid \varkappa(i)=0\}$ denote the counter of total transfer kernels $\operatorname{ker}\left(T_{i}\right)=G$, which is an invariant of the group $G$. In 1980, Chang and Foote [26] proved that, for any odd prime $p$ and for any integer $0 \leq n \leq p+1$, there exist metabelian $p$-groups $G$ having abelianization $G / G^{\prime}$ of type $(p, p)$ such that $\# \mathcal{H}_{0}(G)=n$. However, for $p=2$, there do not exist non-abelian 2-groups $G$ with $G / G^{\prime} \simeq(2,2)$, such that $\# \mathcal{H}_{0}(G) \geq 2$. Such groups must be metabelian of maximal class. Only the elementary abelian 2-group $G=C_{2} \times C_{2}$ has $\# \mathcal{H}_{0}(G)=3$.

In the following concrete examples for the counters $\# \mathcal{H}_{0}(G)$, and also in the remainder of this article, we use identifiers of finite $p$-groups in the SmallGroups Library by Besche, Eick and O’Brien [33] [34].

Example 5.1. For $p=3$, we have the following TKTs $\varkappa=\varkappa(G)$ :

- $\# \mathcal{H}_{0}(G)=0$ for the extra special group $G=\langle 27,4\rangle$ of exponent 9 with $\varkappa=(1,1,1,1)$,

- $\# \mathcal{H}_{0}(G)=1$ for the two groups $G \in\{\langle 243,6\rangle,\langle 243,8\rangle\}$ with $\varkappa \in\{(0,1,2,2),(2,0,3,4)\}$,

- $\# \mathcal{H}_{0}(G)=2$ for the group $G=\langle 243,3\rangle$ with $\varkappa=(0,0,4,3)$,

- $\# \mathcal{H}_{0}(G)=3$ for the group $G=\langle 81,7\rangle$ with $\varkappa=(2,0,0,0)$,

- $\# \mathcal{H}_{0}(G)=4$ for the extra special group $G=\langle 27,3\rangle$ of exponent 3 with $\varkappa=(0,0,0,0)$.

\subsection{Abelianization of Type $\left(p^{2}, p\right)$}

Let $G$ be a $p$-group with abelianization $G / G^{\prime}$ of non-elementary abelian type $\left(p^{2}, p\right)$. Then $G$ possesses $p+1$ maximal subgroups $H_{1, i}<G \quad(1 \leq i \leq p+1)$ of index $\left(G: H_{1, i}\right)=p$, and $p+1$ subgroups $H_{2, i}<G \quad(1 \leq i \leq p+1)$ of index $\left(G: H_{2, i}\right)=p^{2}$. See Figure 1 .

P 5.12. Convention. Suppose that $H_{1, p+1}=\prod_{j=1}^{p+1} H_{2, j}$ is the distinguished maximal subgroup which is the product of all subgroups of index $p^{2}$, and $H_{2, p+1}=\bigcap_{j=1}^{p+1} H_{1, j}$ is the distinguished subgroup of index $p^{2}$ which is the intersection of all maximal subgroups, that is the Frattini subgroup $\Phi(G)$ of $G$.

P 5.13. First layer. For each $1 \leq i \leq p+1$, let $T_{1, i}: G \rightarrow H_{1, i} / H_{1, i}^{\prime}$ be the Artin transfer homomorphism from $G$ to the ab- elianization of $H_{1, i}$.

Definition 5.9. The family $\varkappa_{1, H}(G)=\left(\operatorname{ker}\left(T_{1, i}\right)\right)_{1 \leq i \leq p+1}$ is called the first layer transfer kernel type of $G$ with respect to $H_{1,1} \cdots, H_{1, p+1}$ and $H_{2,1}, \cdots, H_{2, p+1}$, and is identified with $\left(\varkappa_{1}(i)\right)_{1 \leq i \leq p+1}$, where

$$
\varkappa_{1}(i)= \begin{cases}0 & \text { if } \operatorname{ker}\left(T_{1, i}\right)=H_{1, p+1}, \\ j & \text { if } \operatorname{ker}\left(T_{1, i}\right)=H_{2, j} \text { for some } 1 \leq j \leq p+1 .\end{cases}
$$


Remark 5.5. Here, we observe that each first layer transfer kernel is of exponent $p$ with respect to $G^{\prime}$ and consequently cannot coincide with $H_{1, j}$ for any $1 \leq j \leq p$, since $H_{1, i} / G^{\prime}$ is cyclic of order $p^{2}$, whereas $H_{1, p+1} / G^{\prime}$ is bicyclic of type $(p, p)$.

P 5.14. Second layer. For each $1 \leq i \leq p+1$, let $T_{2, i}: G \rightarrow H_{2, i} / H_{2, i}^{\prime}$ be the Artin transfer homomorphism from $G$ to the abelianization of $H_{2, i}$.

Definition 5.10. The family $\varkappa_{2, H}(G)=\left(\operatorname{ker}\left(T_{2, i}\right)\right)_{1 \leq i \leq p+1}$ is called the second layer transfer kernel type of $G$ with respect to $H_{2,1}, \cdots, H_{2, p+1}$ and $H_{1,1} \cdots, H_{1, p+1}$, and is identified with $\left(\varkappa_{2}(i)\right)_{1 \leq i \leq p+1}$, where

$$
\varkappa_{2}(i)= \begin{cases}0 & \text { if } \operatorname{ker}\left(T_{2, i}\right)=G, \\ j & \text { if } \operatorname{ker}\left(T_{2, i}\right)=H_{1, j} \text { for some } 1 \leq j \leq p+1 .\end{cases}
$$

\section{P 5.15. Transfer kernel type.}

Combining the information on the two layers, we obtain the (complete) transfer kernel type

$\varkappa_{H}(G)=\left(\varkappa_{1, H}(G) ; \varkappa_{2, H}(G)\right)$

of the $p$-group $G$ with respect to $H_{1,1}, \cdots, H_{1, p+1}$ and $H_{2,1}, \cdots, H_{2, p+1}$.

Remark 5.6. The distinguished subgroups $H_{1, p+1}$ and $H_{2, p+1}=\Phi(G)$ are unique invariants of $G$ and should not be renumerated. However, independent renumerations of the remaining maximal subgroups $K_{1, i}=H_{1, \tau(i)}(1 \leq i \leq p)$ and the transfers $V_{1, i}=T_{1, \tau(i)}$ by means of a permutation $\tau \in S_{p}$, and of the remaining subgroups $K_{2, i}=H_{2, \sigma(i)}(1 \leq i \leq p)$ of index $p^{2}$ and the transfers $V_{2, i}=T_{2, \sigma(i)}$ by means of a permutation $\sigma \in S_{p}$, give rise to new TKTs $\lambda_{1, K}(G)=\left(\operatorname{ker}\left(V_{1, i}\right)\right)_{1 \leq i \leq p+1}$ with respect to $K_{1,1}, \cdots, K_{1, p+1}$ and $K_{2,1}, \cdots, K_{2, p+1}$, identified with $\left(\lambda_{1}(i)\right)_{1 \leq i \leq p+1}$, where

$$
\lambda_{1}(i)= \begin{cases}0 & \text { if } \operatorname{ker}\left(V_{1, i}\right)=K_{1, p+1}, \\ j & \text { if } \operatorname{ker}\left(V_{1, i}\right)=K_{2, j} \text { for some } 1 \leq j \leq p+1,\end{cases}
$$

and $\lambda_{2, K}(G)=\left(\operatorname{ker}\left(V_{2, i}\right)\right)_{1 \leq i \leq p+1}$ with respect to $K_{2,1}, \cdots, K_{2, p+1}$ and $K_{1,1}, \cdots, K_{1, p+1}$, identified with $\left(\lambda_{2}(i)\right)_{1 \leq i \leq p+1}$, where

$$
\lambda_{2}(i)= \begin{cases}0 & \text { if } \operatorname{ker}\left(V_{2, i}\right)=G, \\ j & \text { if } \operatorname{ker}\left(V_{2, i}\right)=K_{1, j} \text { for some } 1 \leq j \leq p+1 .\end{cases}
$$

It is adequate to view the TKTs $\lambda_{1, K}(G) \sim \varkappa_{1, H}(G)$ and $\lambda_{2, K}(G) \sim \varkappa_{2, H}(G)$ as equivalent. Since we have

$$
K_{2, \lambda_{1}(i)}=\operatorname{ker}\left(V_{1, i}\right)=\operatorname{ker}\left(T_{1, \hat{\tau}(i)}\right)=H_{2, \varkappa_{1}(\hat{\imath}(i))}=K_{2, \tilde{\sigma}^{-1}\left(\varkappa_{1}(\hat{\tau}(i))\right)}
$$

resp.

$$
K_{1, \lambda_{2}(i)}=\operatorname{ker}\left(V_{2, i}\right)=\operatorname{ker}\left(T_{2, \hat{\sigma}(i)}\right)=H_{1, \varkappa_{2}(\hat{\sigma}(i))}=K_{1, \tilde{\tau}^{-1}\left(\varkappa_{2}(\hat{\sigma}(i))\right)},
$$

the relations between $\lambda_{1}$ and $\varkappa_{1}$, resp. $\lambda_{2}$ and $\varkappa_{2}$, are given by $\lambda_{1}=\tilde{\sigma}^{-1} \circ \varkappa_{1} \circ \hat{\tau}$, resp. $\lambda_{2}=\tilde{\tau}^{-1} \circ \varkappa_{2} \circ \hat{\sigma}$. Therefore, $\lambda=\left(\lambda_{1}, \lambda_{2}\right)$ is another representative of the orbit $\varkappa^{S_{p} \times S_{p}}$ of $\varkappa_{1}\left(\varkappa_{1}, \varkappa_{2}\right)$ under the operation

$$
\left((\sigma, \tau),\left(\mu_{1}, \mu_{2}\right)\right) \mapsto\left(\tilde{\sigma}^{-1} \circ \mu_{1} \circ \hat{\tau}, \tilde{\tau}^{-1} \circ \mu_{2} \circ \hat{\sigma}\right)
$$

of the product of two symmetric groups $S_{p} \times S_{p}$ on the set of all pairs of mappings from $\{1, \cdots, p+1\}$ to $\{0, \cdots, p+1\}$, where the extensions $\hat{\pi} \in S_{p+1}$ and $\tilde{\pi} \in S_{p+2}$ of a permutation $\pi \in S_{p}$ are defined by $\hat{\pi}(p+1)=\tilde{\pi}(p+1)=p+1$ and $\tilde{\pi}(0)=0$, and formally

$$
H_{1,0}=K_{1,0}=G, K_{1, p+1}=H_{1, p+1}, \quad H_{2,0}=K_{2,0}=H_{1, p+1} \text {, and } K_{2, p+1}=H_{2, p+1}=\Phi(G) .
$$

Definition 5.11. The orbit $\varkappa(G)=\varkappa^{S_{p} \times S_{p}}$ of any representative $\varkappa=\left(\varkappa_{1}, \varkappa_{2}\right)$ is an invariant of the $p$ group $G$ and is called its transfer kernel type, briefly TKT.

$P$ 5.16. Connections between layers. 
The Artin transfer $T_{2, i}: G \rightarrow H_{2, i} / H_{2, i}^{\prime}$ from $G$ to a subgroup $H_{2, i}$ of index $\left(G: H_{2, i}\right)=p^{2} \quad(1 \leq i \leq p+1)$ is the compositum $T_{2, i}=\tilde{T}_{H_{1, j}, H_{2, i}} \circ T_{1, j}$ of the induced transfer $\tilde{T}_{H_{1, j}, U_{2, i}}: H_{1, j} / H_{1, j}^{\prime} \rightarrow H_{2, i} / H_{2, i}^{\prime}$ from $H_{1, j}$ to $H_{2, i}$ (in the sense of Corollary 7.1 or Corollary 7.3 in the appendix) and the Artin transfer $T_{1, j}: G \rightarrow H_{1, j} / H_{1, j}^{\prime}$ from $G$ to $H_{1, j}$, for any intermediate subgroup $H_{2, i}<H_{1, j}<G$ of index $\left(G: H_{1, j}\right)=p(1 \leq j \leq p+1)$. There occur two situations:

- For the subgroups $H_{2,1}, \cdots, H_{2, p}$ only the distinguished maximal subgroup $H_{1, p+1}$ is an intermediate subgroup.

- For the Frattini subgroup $H_{2, p+1}=\Phi(G)$ all maximal subgroups $H_{1,1}, \cdots, H_{1, p+1}$ are intermediate subgroups.

This causes restrictions for the transfer kernel type $\varkappa_{2}(G)$ of the second layer, since $\operatorname{ker}\left(T_{2, i}\right)=\operatorname{ker}\left(\tilde{T}_{H_{1, j}, H_{2, i}} \circ T_{1, j}\right) \supset \operatorname{ker}\left(T_{1, j}\right)$, and thus

- $\operatorname{ker}\left(T_{2, i}\right) \supset \operatorname{ker}\left(T_{1, p+1}\right)$, for all $1 \leq i \leq p$,

- but even $\operatorname{ker}\left(T_{2, p+1}\right) \supset\left\langle\bigcup_{j=1}^{p+1} \operatorname{ker}\left(T_{1, j}\right)\right\rangle$.

Furthermore, when $G=\langle x, y\rangle$ with $x^{p} \notin G^{\prime}$ and $y^{p} \in G^{\prime}$, an element $x y^{k-1}(1 \leq k \leq p)$ which is of order $p^{2}$ with respect to $G^{\prime}$, can belong to the transfer kernel $\operatorname{ker}\left(T_{2, i}\right)$ only if its $p$ th power $x^{p}$ is contained in $\operatorname{ker}\left(T_{1, j}\right)$, for all intermediate subgroups $H_{2, i}<H_{1, j}<G$, and thus:

- $x y^{k-1} \in \operatorname{ker}\left(T_{2, i}\right)$, for certain $1 \leq i, k \leq p$, enforces the first layer TKT singulet $\varkappa_{1}(p+1)=p+1$,

- but $x y^{k-1} \in \operatorname{ker}\left(T_{2, p+1}\right)$, for some $1 \leq k \leq p$, even specifies the complete first layer TKT multiplet $\varkappa_{1}=\left((p+1)^{p+1}\right)$, that is $\varkappa_{1}(j)=p+1$, for all $1 \leq j \leq p+1$.

\section{Stabilization and Polarization in Descendant Trees}

P 6.1. Theorem 5.4 has proved that it suffices to get an overview of the restricted Artin patterns of metabelian groups $\mathrm{G}$ with $\operatorname{dl}(G)=2$, since groups $\mathrm{G}$ of derived length $\operatorname{dl}(G) \geq 3$ will certainly reveal exactly the same patterns as their metabelianizations $G / G^{\prime \prime}$.

In this section, we present the complete theory of stabilization and polarization of the restricted Artin patterns for an extensive exemplary case, namely for all metabelian 3-groups $G$ with abelianization $G / G^{\prime}$ of type (3,3).

Since the bottom layer, resp. the top layer, of the restricted Artin pattern will be considered in Theorem 6.4 on the commutator subgroup $G^{\prime}$, resp. Theorem 6.5 on the entire group $G$, we first focus on the intermediate layer $\left(\tau_{1}, \varkappa_{1}\right)$ of the maximal subgroups $G^{\prime}<U_{i}<G$.

\subsection{3-Groups of Non-Maximal Class}

P 6.2. We begin with groups $G$ of non-maximal class. Denoting by $m$ the index of nilpotency of $G$, we let $\chi_{j}(G)$ with $2 \leq j \leq m-1$ be the centralizers of two-step factor groups $\gamma_{j}(G) / \gamma_{j+2}(G)$ of the lower central series, that is, the biggest subgroups of $G$ with the property $\left[\chi_{j}(G), \gamma_{j}(G)\right] \leq \gamma_{j+2}(G)$. They form an ascending chain of characteristic subgroups of $G, G^{\prime} \leq \chi_{2}(G) \leq \cdots \leq \chi_{m-2}(G)<\chi_{m-1}(G)=G$, which contain the commutator subgroup. $\chi_{j}(G)$ coincides with $\mathrm{G}$ if and only if $j \geq m-1$. We characterize the smallest two-step centralizer different from the commutator group by an isomorphism invariant $s=s(G)=\min \left\{2 \leq j \leq m-1 \mid \chi_{j}(G)>G^{\prime}\right\}$. According to Nebelung [30], we can assume that G has order $|G|=3^{n}$, class $\mathrm{cl}(G)=c=m-1 \geq 3$, and coclass $\operatorname{cc}(G)=r=e-1=n-c \geq 2$, where $4 \leq m<n \leq 2 m-3$. Let generators of $G=\langle x, y\rangle$ be selected such that $\gamma_{3}(G)=\left\langle y^{3}, x^{3}, \gamma_{4}(G)\right\rangle, x \in G \backslash \chi_{s}(G)$, if $s<m-1$, and $y \in \chi_{s}(G) / G^{\prime}$. Suppose that a fixed ordering of the four maximal subgroups of $\mathrm{G}$ is defined by $U_{i}=\left\langle g_{i}, G^{\prime}\right\rangle$ with $g_{1}=y, g_{2}=x, g_{3}=x y$, and $g_{4}=x y^{-1}$. Let the main commutator of $G$ be declared by $s_{2}=t_{2}=[y, x] \in \gamma_{2}(G)=G^{\prime}$ and higher commutators recursively by $s_{j}=\left[s_{j-1}, x\right], t_{j}=\left[t_{j-1}, y\right] \in \gamma_{j}(G)$ for $j \geq 3$. Starting with the powers $\sigma_{3}=y^{3}, \tau_{3}=x^{3} \in \gamma_{3}(G)$, let $\sigma_{j}=\left[\sigma_{j-1}, x\right], \tau_{j}=\left[\tau_{j-1}, y\right] \in \gamma_{j}(G)$ for $j \geq 4$, and put $\Sigma_{4}=\left\langle\sigma_{4}, \cdots, \sigma_{m-1}\right\rangle, T_{4}=\left\langle\tau_{4}, \cdots, \tau_{e+1}\right\rangle$.

Theorem 6.1. (Non-maximal class.) Let $G$ be a metabelian 3-group of nilpotency class $\operatorname{cl}(G)=c=m-1 \geq 4$ and coclass $\operatorname{cc}(G)=r=e-1 \geq 2$ with abelianization $G / G^{\prime} \simeq(3,3)$. With respect to the projection $\pi(G)=G / \gamma_{c}(G)$ onto the parent, the restricted Artin pattern $\operatorname{AP}_{r}(G)=(\tau(G), \varkappa(G))$ of G reveals

1) a bipolarization and partial stabilization, if $G$ is an interface group with bicyclic last lower central equal to 
the bicyclic first upper central, more precisely

$$
\operatorname{Pol}_{\pi}(G)=\{1,2\}, \operatorname{Stb}_{\pi}(G)=\{3,4\} \Leftrightarrow \gamma_{m-1}(G)=\left\langle\sigma_{m-1}, \tau_{e}\right\rangle=\zeta_{1}(G),
$$

2) a unipolarization and partial stabilization, if $G$ is a core group with cyclic last lower central and bicyclic first upper central, more precisely

$$
\operatorname{Pol}_{\pi}(G)=\{1\}, \operatorname{Stb}_{\pi}(G)=\{2,3,4\} \Leftrightarrow \gamma_{m-1}(G)=\left\langle\sigma_{m-1}\right\rangle<\zeta_{1}(G)=\left\langle\sigma_{m-1}, \tau_{e}\right\rangle,
$$

3) a nilpolarization and total stabilization, if $G$ is a core group with cyclic last lower central equal to the cyclic first upper central, more precisely

$$
\operatorname{Pol}_{\pi}(G)=\varnothing, \operatorname{Stb}_{\pi}(G)=\{1,2,3,4\} \Leftrightarrow \gamma_{m-1}(G)=\left\langle\sigma_{m-1}\right\rangle=\left\langle\tau_{e+1}\right\rangle=\zeta_{1}(G) .
$$

Proof. Theorems 5.1 and 5.2 tell us that for detecting whether stabilization occurs from parent $\pi(G)$ to child $G$, we have to compare the projection kernel $\operatorname{ker}(\pi)$ with the commutator subgroups $U_{i}^{\prime}$ of the four maximal normal subgroups $U_{i} \triangleleft G, 1 \leq i \leq 4$. According to ([35], Cor.3.2, p. 480) these derived subgroups are given by

$$
\begin{aligned}
& U_{1}^{\prime}=\left\langle t_{3}, \tau_{4}, \cdots, \tau_{e+1}\right\rangle, \\
& U_{2}^{\prime}=\left\langle s_{3}, \sigma_{4}, \cdots, \sigma_{m-1}\right\rangle, \\
& U_{3}^{\prime}=\left\langle s_{3} t_{3}, \gamma_{4}(G)\right\rangle, \\
& U_{4}^{\prime}=\left\langle s_{3} t_{3}^{-1}, \gamma_{4}(G)\right\rangle,
\end{aligned}
$$

provided the generators of $G$ are selected as indicated above. On the other hand, the projection kernel $\operatorname{ker}(\pi)=\gamma_{m-1}(G)$ is given by

$$
\operatorname{ker}(\pi)= \begin{cases}\left\langle\sigma_{m-1}, \tau_{e}\right\rangle & \text { if } e=m-1, \\ \left\langle\sigma_{m-1}\right\rangle & \text { if } e<m-1, \zeta_{1}(G)=\left\langle\sigma_{m-1}, \tau_{e}\right\rangle, \\ \left\langle\sigma_{m-1}\right\rangle=\left\langle\tau_{e+1}\right\rangle & \text { if } e<m-1, \zeta_{1}(G)=\left\langle\sigma_{m-1}\right\rangle .\end{cases}
$$

Combining this information with $m-1=c$, we obtain the following results.

- $\operatorname{ker}(\pi)=\gamma_{c}(G) \leq \gamma_{4}(G) \subset U_{i}^{\prime}$ for $3 \leq i \leq 4$ if $c \geq 4$, independently of $3 \leq e \leq m-1$.

- $\operatorname{ker}(\pi)=\left\langle\sigma_{m-1}, \tau_{e}\right\rangle \nsubseteq U_{i}^{\prime}$ for $1 \leq i \leq 2$ if $e=m-1$, which implies $\Sigma_{4} \cap T_{4}=1$.

- $\operatorname{ker}(\pi)=\left\langle\sigma_{m-1}\right\rangle \subset U_{2}^{\prime}$ but $\operatorname{ker}(\pi)=\left\langle\sigma_{m-1}\right\rangle \nsubseteq U_{1}^{\prime}$ if $e<m-1, \quad \zeta_{1}(G)=\left\langle\sigma_{m-1}, \tau_{e}\right\rangle$, which also implies $\Sigma_{4} \cap T_{4}=1$.

- $\operatorname{ker}(\pi)=\left\langle\sigma_{m-1}\right\rangle \subset U_{i}^{\prime}$ for $1 \leq i \leq 2$ if $e<m-1, \quad \zeta_{1}(G)=\left\langle\sigma_{m-1}\right\rangle$, which implies $\Sigma_{4} \cap T_{4}=\left\langle\sigma_{m-1}\right\rangle=\left\langle\tau_{e+1}\right\rangle$.

Taken together, these results justify all claims.

Example 6.1. Generally, the parent $\pi(G)$ of an interface group G ([19], Dfn.3.3, p. 430) with bicyclic last non-trivial lower central is a vertex of a different coclass graph with lower coclass. In the case of a bipolarization ([19], Dfn.3.2, p. 430), which is now also characterized via the Artin pattern by Formula (6.1) for $p=3$, we can express the membership in coclass graphs by the implication: If $G \in \mathcal{G}(p, r)$ with $r \geq 3$, then $\pi(G) \in \mathcal{G}(p, r-1)$. A typical example is the group $G=\langle 2187,77\rangle$ of coclass 3 with parent $\pi(G)=\langle 243,3\rangle \prec G$ of coclass 2 (again with identifiers in the SmallGroups database [33] [34]), where

$$
\tau(\pi(G))=[(9,3),(9,3),(3,3,3),(3,3,3)] \prec \tau(G)=[(9,9),(9,9),(3,3,3),(3,3,3)]
$$

and

$$
\varkappa(G)=(3,4,4,3) \prec \varkappa(\pi(G))=(0,0,4,3) .
$$

In contrast, a core group $G$ ([19], Dfn.3.3, p. 430) with cyclic last non-trivial lower central and its parent $\pi(G)$ are vertices of the same coclass graph. In dependence on the $p$-rank of its centre $\zeta_{1}(G)$, the Artin pattern either shows a unipolarization as in Formula (6.2), if the centre is bicyclic, or a total stabilization as in Formula (6.3), if the centre is cyclic. Typical examples are the group $G=\langle 2187,304\rangle$ with parent $\pi(G)=\langle 729,54\rangle \prec G$, both of coclass 2 , where the Artin pattern shows a unipolarization 


$$
\tau(\pi(G))=[(9,9),(9,3),(9,3),(9,3)] \prec \tau(G)=[(27,9),(9,3),(9,3)),(9,3)]
$$

and

$$
\varkappa(G)=(1,2,3,1) \prec \varkappa(\pi(G))=(0,2,3,1),
$$

and the group $G=\langle 729,45\rangle$ with parent $\pi(G)=\langle 243,4\rangle \prec G$, both of coclass 2, where the Artin pattern shows a total stabilization

$$
\tau(\pi(G))=[(3,3,3),(3,3,3),(9,3),(3,3,3)]=\tau(G)
$$

and

$$
\varkappa(G)=(4,4,4,3)=\varkappa(\pi(G)) .
$$

\section{2. $p$-Groups of Maximal Class}

P 6.3. Next we consider p-groups of maximal class, that is, of coclass $\operatorname{cc}(G)=1$, but now for an arbitrary prime number $p \geq 2$. According to Blackburn [17] and Miech [36], we can assume that $\mathrm{G}$ is a metabelian p-group of order $|G|=p^{n}$ and nilpotency class $\operatorname{cl}(G)=c=m-1$, where $n=m \geq 3$. Then $\mathrm{G}$ is of coclass

$\operatorname{cc}(G)=r=n-c=1$ and the commutator factor group $G / G^{\prime}$ of $\mathrm{G}$ is of type $(p, p)$. The lower central series of $G$ is defined recursively by $\gamma_{1}(G)=G$ and $\gamma_{j}(G)=\left[\gamma_{j-1}(G), G\right]$ for $j \geq 2$, in particular $\gamma_{2}(G)=G^{\prime}$.

The centralizer $\chi_{2}(G)=\left\{g \in G \mid[g, u] \in \gamma_{4}(G)\right.$ for all $\left.u \in \gamma_{2}(G)\right\}$ of the two-step factor group $\gamma_{2}(G) / \gamma_{4}(G)$, that is,

$$
\gamma_{2}(G) / \gamma_{4}(G)=\text { Centralizer }_{G / \gamma_{4}(G)}\left(\gamma_{2}(G) / \gamma_{4}(G)\right)
$$

is the biggest subgroup of $G$ such that $\left[\chi_{2}(G), \gamma_{2}(G)\right] \leq \gamma_{4}(G)$. It is characteristic, contains the commutator subgroup $G^{\prime}$, and coincides with $G$, if and only if $m=3$. Let the isomorphism invariant $k=k(G)$ of $G$ be defined by

$$
\left[\chi_{2}(G), \gamma_{2}(G)\right]=\gamma_{m-k}(G),
$$

where $k=0$ for $3 \leq m \leq 4,0 \leq k \leq m-4$ for $m \geq 5$, and $0 \leq k \leq \min (m-4, p-2)$ for $m \geq p+1$, according to Miech ([36], p. 331).

Suppose that generators of $G=\langle x, y\rangle$ are selected such that $x \in G \backslash \chi_{2}(G)$, if $m \geq 4$, and $y \in \chi_{2}(G) \backslash G^{\prime}$. We define the main commutator $s_{2}=[y, x] \in \gamma_{2}(G)$ and the higher commutators $s_{j}=\left[s_{j-1}, x\right]=s_{j-1}^{x-1} \in \gamma_{j}(G)$ for $j \geq 3$.

The maximal subgroups $U_{i}$ of $G$ contain the commutator subgroup $G^{\prime}$ of $G$ as a normal subgroup of index $p$ and thus are of the shape $U_{i}=\left\langle g_{i}, G^{\prime}\right\rangle$. We define a fixed ordering by $g_{1}=y$ and $g_{i}=x y^{i-2}$ for $2 \leq i \leq p+1$.

Theorem 6.2. (Maximal class.) Let $G$ be a metabelian p-group of nilpotency class $\operatorname{cl}(G)=c=m-1 \geq 3$ and coclass $\operatorname{cc}(G)=r=e-1=1$, which automatically implies an abelianization $G / G^{\prime}$ of type $(p, p)$. With respect to the projection $\pi(G)=G / \gamma_{c}(G)$ onto the parent, the restricted Artin pattern $\operatorname{AP}_{r}(G)=(\tau(G), \varkappa(G))$ of $G$ reveals

1) a unipolarization and partial stabilization, if the first maximal subgroup $U_{1}$ of $G$ is abelian, more precisely

$$
\operatorname{Pol}_{\pi}(G)=\{1\}, \operatorname{Stb}_{\pi}(G)=\{2, \cdots, p+1\} \Leftrightarrow U_{1}^{\prime}=1, k=0,
$$

2) a nilpolarization and total stabilization, if all four maximal subgroups $U_{i}$ of $G$ are non-abelian, more precisely

$$
\operatorname{Pol}_{\pi}(G)=\varnothing, \operatorname{Stb}_{\pi}(G)=\{1, \cdots, p+1\} \Leftrightarrow U_{1}^{\prime}=\left\langle s_{m-k}, \cdots, s_{m-1}\right\rangle=\gamma_{m-k}(G), k \geq 1 .
$$

In both cases, the commutator subgroups of the other maximal normal subgroups of $G$ are given by

$$
U_{i}^{\prime}=\left\langle s_{3}, \cdots, s_{m-1}\right\rangle=\gamma_{3}(G) \text { for } 2 \leq i \leq p+1 .
$$


Proof. We proceed in the same way as in the proof of Theorem 6.1 and compare the projection kernel $\operatorname{ker}(\pi)$ with the commutator subgroups $U_{i}^{\prime}$ of the $p+1$ maximal normal subgroups $U_{i} \triangleleft G, 1 \leq i \leq p+1$. According to ([35], Cor.3.1, p. 476) they are given by

$$
\begin{aligned}
& U_{1}^{\prime}=\left\langle s_{m-k}, \cdots, s_{m-1}\right\rangle=\gamma_{m-k}(G), k \geq 0, \\
& U_{i}^{\prime}=\left\langle s_{3}, \cdots, s_{m-1}\right\rangle=\gamma_{3}(G) \text { for } 2 \leq i \leq p+1,
\end{aligned}
$$

if the generators of $G$ are chosen as indicated previously. The cyclic projection kernel is given uniformly by

$$
\operatorname{ker}(\pi)=\gamma_{m-1}(G)=\left\langle s_{m-1}\right\rangle=\zeta_{1}(G) .
$$

Using the relation $m-1=c$, we obtain the following results.

- $\operatorname{ker}(\pi)=\gamma_{c}(G) \leq \gamma_{3}(G)=U_{i}^{\prime}$ for $2 \leq i \leq p+1$ if $c \geq 3$.

- $\operatorname{ker}(\pi)=\gamma_{m-1}(G) \leq \gamma_{m-k}(G)=U_{1}^{\prime}$ if and only if $m-1 \geq m-k$, that is, $k \geq 1$.

The claims follow by applying Theorems 5.1 and 5.2.

Example 6.2. For $p=5$, typical examples are the group $G=\langle 625,8\rangle$ with parent $\pi(G)=\langle 125,3\rangle \prec G$, both of coclass 1, where the Artin pattern shows a unipolarization ([19], Dfn.3.1, p. 413)

$$
\tau(\pi(G))=[(5,5,5),(5,5),(5,5),(5,5),(5,5),(5,5)] \prec \tau(G)=[(5,5),(5,5),(5,5),(5,5),(5,5),(5,5)]
$$

and

$$
\varkappa(G)=(1,0,0,0,0,0) \prec \varkappa(\pi(G))=(0,0,0,0,0,0),
$$

and the group $G=\langle 3125,33\rangle$ with parent $\pi(G)=\langle 625,7\rangle \prec G$, both of coclass 1 , where the Artin pattern shows a total stabilization

$$
\tau(\pi(G))=[(5,5,5),(5,5),(5,5),(5,5),(5,5),(5,5)]=\tau(G)
$$

and

$$
\varkappa(G)=(0,0,0,0,0,0)=\varkappa(\pi(G)) .
$$

\subsection{Extreme Interfaces of $p$-Groups}

P 6.4. Finally, what can be said about the extreme cases (excluded in Theorems 6.1 and 6.2) of non-abelian p-groups having the smallest possible nilpotency class $c=3$ for coclass $r \geq 2$ and $c=2$ for coclass $r=1$ ? In these particular situations, the answers can be given for arbitrary prime numbers $p \geq 2$.

Theorem 6.3. Let $G$ be a metabelian p-group with abelianization $G / G^{\prime}$ of type $(p, p)$.

1) If $G$ is of coclass $\operatorname{cc}(G) \geq 2$ and nilpotency class $\operatorname{cl}(G)=3$, then $p \geq 3$ must be odd and the coclass must be $\operatorname{cc}(G)=2$ exactly.

2) If $G$ is of coclass $\operatorname{cc}(G)=1$ and nilpotency class $\operatorname{cl}(G)=2$, then $G$ is an extra special $p$-group of order $p^{3}$ and exponent $p$ or $p^{2}$.

In both cases, there occurs a total polarization and no stabilization at all, more explicitly

$$
\operatorname{Pol}_{\pi}(G)=\{1, \cdots, p+1\}, \operatorname{Stb}_{\pi}(G)=\varnothing .
$$

Proof. Suppose that $G$ is a metabelian $p$-group with $G / G^{\prime} \simeq(p, p)$.

1) According to O. Taussky [37], a 2-group $G$ with abelianization $G / G^{\prime}$ of type $(2,2)$ must be of coclass $\operatorname{cc}(G)=1$. Consequently, $\operatorname{cc}(G) \geq 2$ implies $p \geq 3$.

Since the minimal nilpotency class $c$ of a non-abelian group with coclass $r \geq 1$ is given by $c=r+1$, the case $\operatorname{cl}(G)=3$ cannot occur for $\operatorname{cc}(G) \geq 3$.

So we are considering metabelian $p$-groups $G$ with $G / G^{\prime} \simeq(p, p)$, nilpotency class $\operatorname{cl}(G)=3$ and coclass $\operatorname{cc}(G)=2$ for odd $p \geq 3$, which form the stem of the isoclinism family $\Phi_{6}$ in the sense of P. Hall. According to ([19], Lem.3.1, p. 446), the commutator subgroups $U_{i}^{\prime}$ of the maximal subgroups $U_{i}<G$ are cyclic of degree $p$, for such a group $G \in \Phi_{6}(0)$. However, the kernel of the parent projection $\pi$ is the bicyclic group $\operatorname{ker}(\pi)=\gamma_{3}(G)$ of type $(p, p)$ ([19], §3.5, p. 445), which cannot be contained in any of the cyclic $U_{i}^{\prime}$ with $1 \leq i \leq p+1$. 
2) According to ([35], Cor.3.1, p. 476), the commutator subgroups $U_{i}^{\prime}$ of all maximal subgroups $U_{i}<G$ are trivial, for a metabelian $p$-group $G$ of coclass $\operatorname{cc}(G)=1$ and nilpotency class $m-1=c=\operatorname{cl}(G)=2$, which implies $k=0$. Thus, the kernel of the parent projection $\operatorname{ker}(\pi)=\gamma_{2}(G)=\left\langle s_{2}\right\rangle$ is not contained in any $U_{i}^{\prime}=1$.

In both cases, the final claim is a consequence of the Theorems 5.1 and 5.2.

Example 6.3. For $p=3$, a typical example for the interface between groups of coclass 2 and 1 is the group $G=\langle 243,9\rangle$ of coclass 2 with parent $\pi(G)=\langle 27,3\rangle \prec G$ of coclass 1 , where the Artin pattern shows a total polarization

$$
\tau(\pi(G))=[(9,3),(9,3),(9,3),(9,3)] \prec[(3,3),(3,3),(3,3),(3,3)]=\tau(G)
$$

and

$$
\varkappa(G)=(2,1,4,3) \prec \varkappa(\pi(G))=(0,0,0,0) .
$$

For $p=2$, a typical example for the interface between non-abelian and abelian groups is the extra special quaternion group $Q=\langle 8,4\rangle$ with parent $\pi(Q)=\langle 4,2\rangle \prec Q$ both of coclass 1, where the Artin pattern shows a total polarization

$$
\tau(\pi(Q))=[(2),(2),(2)] \prec \tau(Q)=[(4),(4),(4)]
$$

and

$$
\varkappa(Q)=(1,2,3) \prec \varkappa(\pi(Q))=(0,0,0) .
$$

Summarizing, we can say that the last three Theorems 6.1, 6.2, and 6.3 underpin the fact that Artin transfer patterns provide a marvellous tool for classifying finite $p$-groups.

\subsection{Bottom and Top Layer of the Artin Pattern}

P 6.5. We conclude this section with supplementary general results concerning the bottom layer and top layer of the restricted Artin pattern.

Theorem 6.4. (Bottom layer.) The type of the commutator subgroup $G^{\prime}$ can never remain stable for a metabelian vertex $G \in \mathcal{T}$ of a descendant tree $\mathcal{T}$ with respect to the lower central series, lower exponent-p central series, or derived series. The kernel of $T_{G, G^{\prime}}: G \rightarrow G^{\prime} / G^{\prime \prime}$ is equal to $G$ (Principal Ideal Theorem).

Proof. All possible kernels $\operatorname{ker}(\pi)=\gamma_{c}(G)>1$, resp. $\operatorname{ker}(\pi)=\mathrm{P}_{c-1}(G)>1$, resp. $\operatorname{ker}(\pi)=G^{(d-1)}>1$, of the parent projections $\pi$ are non trivial, and can therefore never be contained in the trivial second derived subgroup G". According to Theorem 5.1, the type of the commutator subgroup $G^{\prime}$ cannot be stable. The Principal Ideal Theorem is due to Furtwängler [38] and is also proved in ([13] Thm.10.18, p. 313).

Example 6.4. In Example 6.1, we point out that the group $G=\langle 729,45\rangle$ with cyclic centre and its parent $\pi(G)=\langle 243,4\rangle \prec G$, both of coclass 2 , cannot be distinguished by their TTT

$$
\tau_{1}(\pi(G))=[(3,3,3),(3,3,3),(9,3),(3,3,3)]=\tau_{1}(G)
$$

and TKT

$$
\varkappa_{1}(G)=(4,4,4,3)=\varkappa_{1}(\pi(G)),
$$

due to a total stabilization of the restricted Artin pattern as in Formula (6.3). However, the type of their commutator subgroup (the second layer of their TTT) admits a distinction, since

$$
\tau_{2}(\pi(G))=(3,3,3) \prec(9,3,3)=\tau_{2}(G) .
$$

Theorem 6.5. (Top layer.) In a descendant tree $\mathcal{T}$ with respect to the lower central series or derived series, the type of the abelianization $G / G^{\prime}$ of $G \in \mathcal{T}$ remains stable. The kernel of $T_{G . G}: G \rightarrow G / G^{\prime}$ is equal to $G^{\prime}$.

Proof. This follows from Theorem 5.1, since even the maximal possible kernel $\operatorname{ker}(\pi)=\gamma_{2}(G)=G^{\prime}$, resp. $\operatorname{ker}(\pi)=G^{(1)}=G^{\prime}$, of the parent projections $\pi$ is contained in the commutator subgroup $G^{\prime}$ of $G$.

We briefly emphasize the different behaviour of trees where parents are defined with the lower exponent- $p$ 
central series.

Theorem 6.6. In a descendant tree $\mathcal{T}$ with respect to the lower exponent-p central series, only the p-rank of the abelianization $r_{p}\left(G / G^{\prime}\right)$ of the vertices $G \in \mathcal{T}$ remains stable.

Proof. Denote by $\rho:=r_{p}\left(G / G^{\prime}\right)$ the $p$-rank of the abelianization of $G$. According to Theorem 5.1, the maximal possible kernel $\operatorname{ker}(\pi)=\mathrm{P}_{1}(G)=\Phi(G)$ of the parent projections $\pi$ is the Frattini subgroup which is contained in all maximal subgroups $U_{i}$ of $G$. According to Proposition 5.1, the map $\pi$ induces a bijection between the sets of maximal subgroups of the child $G$ and the parent $\pi(G)$, whose cardinality is given by $\frac{p^{\rho}-1}{p-1}$. Consequently, we have $r_{p}\left(\pi(G) / \pi(G)^{\prime}\right)=\rho=r_{p}\left(G / G^{\prime}\right)$.

\section{Acknowledgements}

The author would like to express his heartfelt gratitude to Professor Mike F. Newman from the Australian National University in Canberra, Australian Capital Territory, for his continuing encouragement and interest in our endeavour to strengthen the bridge between group theory and class field theory which was initiated by the ideas of Emil Artin, and for his untiring willingness to share his extensive knowledge and expertise and to be a source of advice in difficult situations.

We also gratefully acknowledge that our research is supported by the Austrian Science Fund (FWF): P 26008N25.

\section{References}

[1] Artin, E. (1927) Beweis des allgemeinen Reziprozitätsgesetzes. Abhandlungen aus dem Mathematischen Seminar der Universität Hamburg, 5, 353-363. http://dx.doi.org/10.1007/BF02952531

[2] Artin, E. (1929) Idealklassen in Oberkörpern und allgemeines Reziprozitätsgesetz. Abhandlungen aus dem Mathematischen Seminar der Universität Hamburg, 7, 46-51. http://dx.doi.org/10.1007/BF02941159

[3] Mayer, D.C. (2015) Periodic Bifurcations in Descendant Trees of Finite p-Groups. Advances in Pure Mathematics, 5, 162-195. http://dx.doi.org/10.4236/apm.2015.54020

[4] Newman, M.F. (1977) Determination of Groups of Prime-Power Order. In: Bryce, R.A., Cossey, J. and Newman, M.F., Eds., Group Theory, Lecture Notes in Math, Vol.573, Springer, Berlin, 73-84.

[5] O’Brien, E.A. (1990) The p-Group Generation Algorithm. Journal of Symbolic Computation, 9, 677-698. http://dx.doi.org/10.1016/S0747-7171(08)80082-X

[6] Schur, I. (1902) Neuer Beweis eines Satzes über endliche Gruppen. Sitzungsberichte der Königlich Preussischen Akademie der Wissenschaften zu Berlin, 42, 1013-1019.

[7] Hall Jr., M. (1999) The Theory of Groups. AMS Chelsea Publishing, American Mathematical Society, Providence.

[8] Huppert, B. (1979) Endliche Gruppen I. Grundlehren der mathematischen Wissenschaften, Vol. 134. Springer-Verlag, Berlin, Heidelberg and New York.

[9] Gorenstein, D. (2012) Finite Groups. AMS Chelsea Publishing, American Mathematical Society, Providence.

[10] Aschbacher, M. (1986) Finite Group Theory. Cambridge Studies in Advanced Mathematics, Vol. 10. Cambridge University Press, Cambridge.

[11] Doerk, K. and Hawkes, T. (1992) Finite Soluble Groups, de Gruyter Expositions in Mathematics, Vol.4. Walter de Gruyter, Berlin and New York. http://dx.doi.org/10.1515/9783110870138

[12] Smith, G. and Tabachnikova, O. (2000) Topics in Group Theory. Springer Undergraduate Mathematics Series (SUMS), Springer-Verlag, London.

[13] Isaacs, I.M. (2011) Finite Group Theory. Graduate Studies in Mathematics, Vol.92. American Mathematical Society, Providence.

[14] Bourbaki, N. (2007) Éléments de mathématique, Livre 2, Algèbre. Springer-Verlag, Berlin Heidelberg.

[15] Olsen, J. (2010) The Transfer Homomorphism. Math 434. University of Puget Sound, Tacoma, WA. http://buzzard.ups.edu/courses/2010spring/projects/olsen-transfer-homomorphism-ups-434-2010.pdf

[16] Hasse, H. (1932) Bericht über neuere Untersuchungen und Probleme aus der Theorie der algebraischen Zahlkörper, Teil II: Reziprozitätsgesetz. Jahresbericht der Deutschen Mathematiker-Vereinigung, 6, 1-204.

[17] Blackburn, N. (1958) On a Special Class of p-Groups. Acta Mathematica, 100, 45-92. 
http://dx.doi.org/10.1007/BF02559602

[18] Mayer, D.C. (2015) Index-p abelianization data of p-class tower groups. Advances in Pure Mathematics, 5, $286-313$. http://dx.doi.org/10.4236/apm.2015.55029

[19] Mayer, D.C. (2011) The Distribution of Second p-Class Groups on Coclass Graphs. Journal de Théorie des Nombres de Bordeaux, 25, 401-456.

[20] Mayer, D.C. (2014) Principalization Algorithm via Class Group Structure. Journal de Théorie des Nombres de Bordeaux, 26, 415-464. http://dx.doi.org/10.5802/jtnb.874

[21] Mayer, D.C. (2015) Periodic Sequences of p-Class Tower Groups. Journal of Applied Mathematics and Physics, 3, 746-756. http://dx.doi.org/10.4236/jamp.2015.37090

[22] Mayer, D.C. (2015) New Number Fields with Known p-Class Tower. 22nd Czech and Slovak International Conference on Number Theory, Liptovský Ján, 31 August-4 September 2015.

[23] Bush, M.R. and Mayer, D.C. (2015) 3-Class Field Towers of Exact Length 3. Journal of Number Theory 147, $766-777$. http://dx.doi.org/10.1016/j.jnt.2014.08.010

[24] Scholz, A. and Taussky, O. (1934) Die Hauptideale der kubischen Klassenkörper imaginär quadratischer Zahlkörper: ihre rechnerische Bestimmung und ihr Einfluß auf den Klassenkörperturm. Journal für die reine und angewandte Mathematik, 171, 19-41.

[25] Chang, S.M. (1977) Capitulation Problems in Algebraic Number Fields. Ph.D. Thesis, University of Toronto, Toronto.

[26] Chang, S.M. and Foote, R. (1980) Capitulation in Class Field Extensions of Type (p,p). Canadian Journal of Mathematics, 32, 1229-1243. http://dx.doi.org/10.4153/CJM-1980-091-9

[27] Heider, F.-P. and Schmithals, B. (1982) Zur Kapitulation der Idealklassen in unverzweigten primzyklischen Erweiterungen. Journal für die reine und angewandte Mathematik., 336, 1-25.

[28] Brink, J.R. (1984) The Class Field Tower for Imaginary Quadratic Number Fields of Type (3,3). Dissertation, Ohio State University, Columbus.

[29] Brink, J.R. and Gold, R. (1987) Class Field Towers of Imaginary Quadratic Fields. Manuscripta Mathematica, 57, 425-450.

[30] Nebelung, B. (1989) Klassifikation metabelscher 3-Gruppen mit Faktorkommutatorgruppe vom Typ $(3,3)$ und Anwendung auf das Kapitulationsproblem. Inaugural Dissertation, Universität zu Köln, Cologne.

[31] Mayer, D.C. (1990) Principalization in Complex $S_{3}$-Fields. Congressus Numerantium, 80, 73-87.

[32] Mayer, D.C. (2012) Transfers of Metabelian p-Groups. Monatshefte für Mathematik., 166, 467-495.

[33] Besche, H.U., Eick, B. and O’Brien, E.A. (2002) A Millennium Project: Constructing Small Groups. International Journal of Algebra and Computation, 12, 623-644. http://dx.doi.org/10.1142/S0218196702001115

[34] Besche, H.U., Eick, B. and O’Brien, E.A. (2005) The Small Groups Library-A Library of Groups of Small Order. An Accepted and Refereed GAP 4 Package, Available also in MAGMA.

[35] Mayer, D.C. (2012) The Second p-class Group of a Number Field. International Journal of Number Theory, 8, 471-505. http://dx.doi.org/10.1142/S179304211250025X

[36] Miech, R.J. (1970) Metabelian p-Groups of Maximal Class. Transactions of the American Mathematical Society, 152, 331-373.

[37] Taussky, O. (1937) A Remark on the Class Field Tower. Journal London Mathematical Society, S1-12, 82-85. http://dx.doi.org/10.1112/jlms/s1-12.1.82

[38] Furtwängler, Ph. (1929) Beweis des Hauptidealsatzes für die Klassenkörper algebraischer Zahlkörper. Abhandlungen aus dem Mathematischen Seminar der Universität Hamburg, 7, 14-36. http://dx.doi.org/10.1007/BF02941157

[39] Lang, S. (1980) Algebra. World Student Series, Addison-Wesley Publishing Company, Reading.

[40] Boston, N., Bush, M.R. and Hajir, F. (2014) Heuristics for p-Class Towers of Imaginary Quadratic Fields. http://arxiv.org/abs/1111.4679 


\section{Appendix: Induced Homomorphism between Quotient Groups}

Throughout this appendix, let $\phi: G \rightarrow H$ be a homomorphism from a source group (domain) $G$ to a target group (codomain) $H$.

\section{A.1. Image, Pre-Image and Kernel}

P 7.1. First, we recall some basic facts concerning the image and pre-image of normal subgroups and the kernel of the homomorphism $\phi$.

Lemma 7.1. Suppose that $U \leq G$ and $V \leq H$ are subgroups, and $x, y \in G$ are elements.

1) If $U \unlhd G$ is a normal subgroup of $G$, then its image $\phi(U) \unlhd \phi(G)$ is a normal subgroup of the (total) image $\operatorname{im}(\phi)=\phi(G)$.

2) If $V \unlhd \phi(G)$ is a normal subgroup of the image $\operatorname{im}(\phi)=\phi(G)$, then the pre-image $\phi^{-1}(V) \unlhd G$ is a normal subgroup of $G$.

In particular, the kernel $\operatorname{ker}(\phi)=\phi^{-1}(1) \unlhd G$ of $\phi$ is a normal subgroup of $G$.

3) If $\phi(x)=\phi(y)$, then there exists an element $k \in \operatorname{ker}(\phi)$ such that $x=y \cdot k$.

4) If $\phi(x) \in \phi(U)$, then $x \in U \cdot \operatorname{ker}(\phi)$, i.e., the pre-image of the image satisfies

$$
U \leq \phi^{-1}(\phi(U))=U \cdot \operatorname{ker}(\phi)=\operatorname{ker}(\phi) \cdot U \text {. }
$$

5) Conversely, the image of the pre-image is given by

$$
\phi\left(\phi^{-1}(V)\right)=\phi(G) \cap V \leq V .
$$

The situation of Lemma 7.1 is visualized by Figure 4, where we briefly write $K:=\operatorname{ker}(\phi)=\phi^{-1}(1)$ and $I:=\operatorname{im}(\phi)=\phi(G)$.

Remark 7.1. Note that, in the first statement of Lemma 7.1, we cannot conclude that $\phi(U) \unlhd H$ is a normal subgroup of the target group $H$, and in the second statement of Lemma 7.1, we need not require that $V \unlhd H$ is a normal subgroup of the target group $H$.

Proof. 1) If $U \unlhd G$, then $x^{-1} U x=U$ for all $x \in G$, and thus $\phi(x)^{-1} \phi(U) \phi(x)=\phi\left(x^{-1} U x\right)=\phi(U)$ for all $\phi(x) \in \phi(G)$, i.e., $\phi(U) \unlhd \phi(G)$.

2) If $V \unlhd \phi(G)$, then $(\forall x \in G) \phi(x)^{-1} V \phi(x) \subseteq V$, that is, $(\forall x \in G \forall v \in V) \phi(x)^{-1} v \phi(x) \in V$. In particular, we have $\left(\forall x \in G \forall u \in \phi^{-1}(V)\right) \phi\left(x^{-1} u x\right)=\phi(x)^{-1} \phi(u) \phi(x) \in V$, i.e., $\quad(\forall x \in G) x^{-1} \phi^{-1}(V) x \subseteq \phi^{-1}(V)$, and consequently $\phi^{-1}(V) \unlhd G$.

To prove the claim for the kernel, we put $V:=1 \unlhd G$. 27).

3) If $\phi(x)=\phi(y)$, then $\phi\left(y^{-1} x\right)=\phi(y)^{-1} \phi(x)=1$, and thus $y^{-1} x=k \in \operatorname{ker}(\phi)$. (See also [7], Thm.2.2.1, p.

4) If $\phi(x) \in \phi(U)$, then $(\exists u \in U) \phi(x)=\phi(u)$, and thus $(\exists u \in U \exists k \in \operatorname{ker}(\phi)) x=u \cdot k$, by (3). This shows $\phi^{-1}(\phi(U)) \subseteq U \cdot \operatorname{ker}(\phi)$, and the opposite inclusion is obvious.

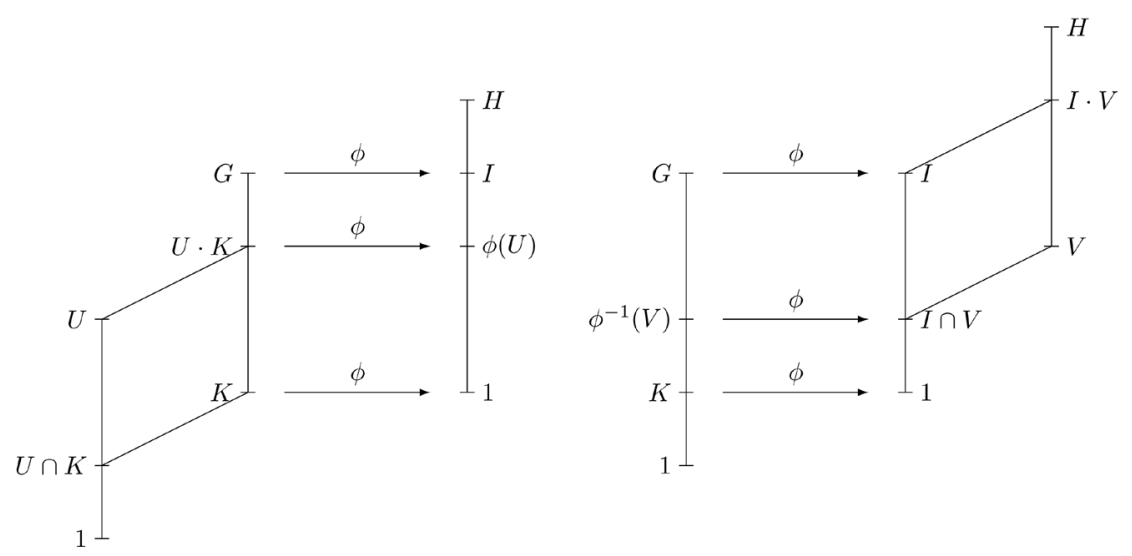

Figure 4. Kernel, image and pre-image under a homomorphism $\phi$. 
Finally, since $\operatorname{ker}(\phi)$ is normal, we have $U \cdot \operatorname{ker}(\phi)=\operatorname{ker}(\phi) \cdot U$.

5) This is a consequence of the properties of the set mappings $\phi^{-1}$ and $\phi$ associated with the homomorphism $\phi$.

\section{A.2. Criteria for the Existence of the Induced Homomorphism}

P 7.2. Now we state the central theorem which provides the foundation for lots of useful applications. It is the most general version of a series of related theorems, which is presented in Bourbaki ([14], Chap.1), Structures algëriques, Prop.5, p. A I.35]. Weaker versions will be given in the subsequent corollaries.

Theorem 7.1. (Main Theorem)

Suppose that $U \unlhd G$ is a normal subgroup of $G$ and $V \unlhd H$ is a normal subgroup of $H$. Let $\omega_{U}: G \rightarrow G / U$ and $\omega_{V}: H \rightarrow H / V$ denote the canonical projections onto the quotients.

- The following three conditions for the homomorphism $\phi: G \rightarrow H$ are equivalent.

1) There exists an induced homomorphism $\tilde{\phi}: G / U \rightarrow H / V$ such that $\tilde{\phi} \circ \omega_{U}=\omega_{V} \circ \phi$, that is,

$$
\tilde{\phi}(x U)=\phi(x) V \quad \text { for all } x \in G \text {. }
$$

2) $\phi(U) \subseteq V$.

3) $U \subseteq \phi^{-1}(V)$.

- If the induced homomorphism $\tilde{\phi}$ of the quotients exists, then it is determined uniquely by $\phi$, and its kernel, image and cokernel are given by

$$
\begin{aligned}
& \operatorname{ker}(\tilde{\phi})=\phi^{-1}(V) / U \supseteq(U \cdot \operatorname{ker}(\phi)) / U, \\
& \operatorname{im}(\tilde{\phi})=(V \cdot \phi(G)) / V, \\
& \operatorname{coker}(\tilde{\phi}) \simeq H /(V \cdot \phi(G)), \text { if } V \cdot \phi(G) \unlhd H .
\end{aligned}
$$

In particular, $\tilde{\phi}$ is a monomorphism if and only if $U=\phi^{-1}(V)$.

Moreover, $\tilde{\phi}$ is an epimorphism if and only if $H=V \cdot \phi(G)$.

In particular, $\tilde{\phi}$ is certainly an epimorphism if $\phi$ is onto.

We summarize the criteria for the existence of the induced homomorphism in a formula:

$$
(\exists \tilde{\phi}: G / U \rightarrow H / V) \tilde{\phi} \circ \omega_{U}=\omega_{V} \circ \phi \Leftrightarrow \phi(U) \subseteq V \Leftrightarrow U \subseteq \phi^{-1}(V) .
$$

The situation of Theorem 7.1 is shown in the commutative diagram of Figure 5.

Remark 7.2. If the normal subgroup $U \unlhd G$ in the assumptions of Theorem 7.17 is taken as $U:=\phi^{-1}(V)$, then the induced homomorphism $\tilde{\phi}$ exists automatically and is a monomorphism.

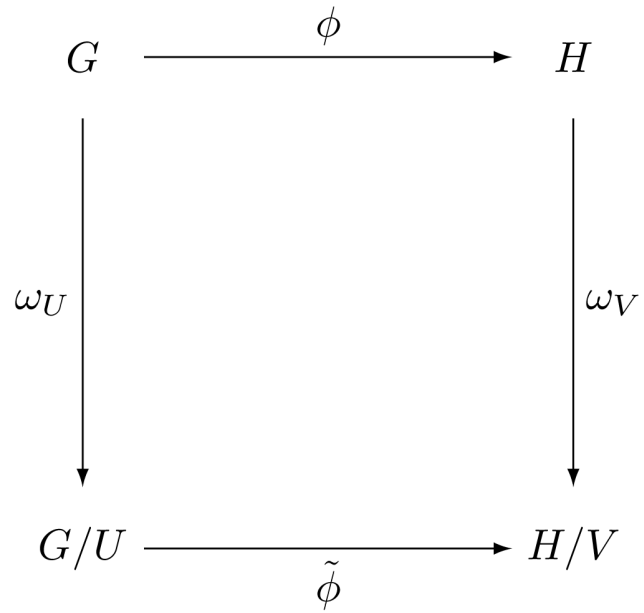

Figure 5. Induced homomorphism $\tilde{\phi}$ of quotients. 
Note that $U=\phi^{-1}(V)$ does not imply $\phi(U)=V$ but only $\phi(U) \subseteq V$, if $\phi$ is not an epimorphism. Similarly, $\phi(U)=V$ does not imply $U=\phi^{-1}(V)$ but only $U \subseteq \phi^{-1}(V)$, if $\phi$ is not a monomorphism. Proof.

- (1) $\Rightarrow$ (2): If there exists a homomorphism $\tilde{\phi}: G / U \rightarrow H / V$ such that $\tilde{\phi}(x U)=\phi(x) V$ for all $x \in G$, then, for any $x \in U$, we have $x U=U=1_{G / U}$, and thus $\phi(x) V=\tilde{\phi}(x U)=\tilde{\phi}\left(1_{G / U}\right)=1_{H / V}=V$, which means $\phi(x) \in V$. It follows that $\phi(U) \subseteq V$.

(2) $\Rightarrow(1)$ : If $\phi(U) \subseteq V$, then the image $\tilde{\phi}(x U)$ of the coset $x U \in G / U$ under $\tilde{\phi}$ is independent of the representative $x \in G$ : If $x U=y U$ for $x, y \in G$, then $y^{-1} x \in U$ and thus $\phi(y)^{-1} \phi(x)=\phi\left(y^{-1} x\right) \in \phi(U) \subseteq V$. Consequently, we have $\tilde{\phi}(x U)=\phi(x) V=\phi(y) V=\tilde{\phi}(y U)$. Furthermore, $\tilde{\phi}$ is a homomorphism, since

$$
\tilde{\phi}(x U \cdot y U)=\tilde{\phi}(x y U)=\phi(x y) V=\phi(x) \phi(y) V=\phi(x) V \cdot \phi(y) V=\tilde{\phi}(x U) \cdot \tilde{\phi}(y U) .
$$

(2) $\Rightarrow$ (3): If $\phi(U) \subseteq V$, then $U \subseteq \phi^{-1}(\phi(U)) \subseteq \phi^{-1}(V)$.

(3) $\Rightarrow$ (2): If $U \subseteq \phi^{-1}(V)$, then $\phi(U) \subseteq \phi\left(\phi^{-1}(V)\right) \subseteq V$.

- The image of any $x U \in G / U$ under $\tilde{\phi}$ is determined uniquely by $\phi$, since $\tilde{\phi}(x U)=\phi(x) V$. The kernel of $\tilde{\phi}$ is given by $\operatorname{ker}(\tilde{\phi})=\tilde{\phi}^{-1}\left(1_{H / V}\right)=\tilde{\phi}^{-1}(V)$, and for $x \in G$ we have

$$
x U \in \tilde{\phi}^{-1}(V) \Leftrightarrow \phi(x) V=\tilde{\phi}(x U)=V \Leftrightarrow \phi(x) \in V \Leftrightarrow x \in \phi^{-1}(V),
$$

that is $\operatorname{ker}(\tilde{\phi})=\phi^{-1}(V) / U$, which clearly contains $\phi^{-1}(\phi(U)) / U=(U \cdot \operatorname{ker}(\phi)) / U$, since $\phi(U) \subseteq V$.

The cokernel of $\tilde{\phi}$ is given by $\operatorname{coker}(\tilde{\phi})=(H / V) / \tilde{\phi}(G / U)=H / V /(V \cdot \phi(G) / V) \simeq H /(V \cdot \phi(G))$, if $V \cdot \phi(G) \unlhd H$.

Finally, if $\phi$ is an epimorphism, then $\tilde{\phi} \circ \omega_{G}=\omega_{H} \circ \phi$ is also an epimorphism, which forces the terminal map $\tilde{\phi}$ to be an epimorphism.

\section{A.3. Factorization through a Quotient}

P 7.3. Theorem 7.1 can be used to derive numerous special cases. Usually it suffices to consider the quotient group $G / U$ corresponding to a normal subgroup $\mathrm{U}$ of the source group $\mathrm{G}$ of the homomorphism $\phi: G \rightarrow H$ and to view the target group $\mathrm{H}$ as the trivial quotient $\mathrm{H} / 1$. In this weaker form, the existence criterion for the induced homomorphism occurs in Lang's book ([39], p. 17).

Corollary 7.1. (Factorization through a quotient)

Suppose $U \unlhd G$ is a normal subgroup of $G$ and $\omega: G \rightarrow G / U$ denotes the natural epimorphism onto the quotient.

If $U \leq \operatorname{ker}(\phi)$, then there exists a unique homomorphism $\tilde{\phi}: G / U \rightarrow H$ such that $\tilde{\phi} \circ \omega=\phi$, that is, $\tilde{\phi}(x U)=\phi(x)$ for all $x \in G$.

Moreover, the kernel of $\tilde{\phi}$ is given by $\operatorname{ker}(\tilde{\phi})=\operatorname{ker}(\phi) / U$.

Again we summarize the criterion in a formula:

$$
(\exists \tilde{\phi}: G / U \rightarrow H) \tilde{\phi} \circ \omega=\phi \Leftrightarrow \phi(U)=1 \Leftrightarrow U \leq \operatorname{ker}(\phi) .
$$

In this situation the homomorphism $\phi$ is said to factor or factorize through the quotient $G / U$ via the canonical projection $\omega$ and the induced homomorphism $\tilde{\phi}$.

The scenario of Corollary 7.1 is visualized by Figure 6 .

Proof. The claim is a consequence of Theorem 7.1 in the special case that $V:=1$ is the trivial group. The equivalent conditions for the existence of the induced homomorphism $\tilde{\phi}$ are $\phi(U)=1$ resp.

$U \subseteq \phi^{-1}(1)=\operatorname{ker}(\phi)$.

Remark 7.3. Note that the well-known isomorphism theorem (sometimes also called homomorphism theorem) is a special case of Corollary 7.1. If we put $U_{\tilde{\phi}}:=\operatorname{ker}(\phi)$ and if we assume that $\phi$ is an epimorphism with $\phi(G)=H$, then the induced homomorphism $\tilde{\phi}: G / \operatorname{ker}(\phi) \rightarrow \phi(G)$ is an isomorphism, since $\operatorname{ker}(\tilde{\phi})=\operatorname{ker}(\phi) / U \simeq 1$.

In this weakest form,

$$
G / \operatorname{ker}(\phi) \simeq \operatorname{im}(\phi)
$$




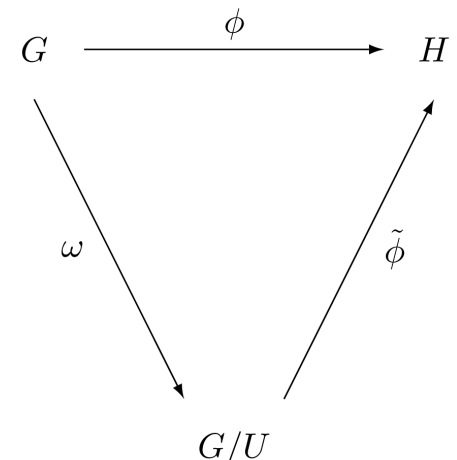

Figure 6. Homomorphism $\phi$ factorized through a quotient.

actually without any additional assumptions being required, the existence theorem for the induced homomorphism appears in almost every standard text book on group theory or algebra, e.g., ([7], Thm.2.3.2, p. 28) and ([13], Thm.X.18, p. 339).

\section{A.4. Application to Series of Characteristic Subgroups}

P 7.4. The normal subgroup $U \unlhd G$ in the assumptions of Corollary 7.1 can be specialized to various characteristic subgroups of $\mathrm{G}$ for which the condition $U \subseteq \operatorname{ker}(\phi)$ can be expressed differently, namely by invariants of series of characteristic subgroups.

Corollary 7.2. The homomorphism $\phi: G \rightarrow H$ can be factorized through various quotients of $G$ in the following way. Let $n$ be a positive integer and $p$ be a prime number.

1) $\phi$ factors through the nth derived quotient $G / G^{(n)}$ if and only if the derived length of $\phi(G)$ is bounded by $\operatorname{dl}(\phi(G)) \leq n$.

2) $\phi$ factors through the nth lower central quotient $G / \gamma_{n}(G)$ if and only if the nilpotency class of $\phi(G)$ is bounded by $\operatorname{cl}(\phi(G)) \leq n-1$.

3) $\phi$ factors through the nth lower exponent-p central quotient $G / P_{n}(G)$ if and only if the p-class of $\phi(G)$ is bounded by $\operatorname{cl}_{p}(\phi(G)) \leq n$.

We summarize these criteria in terms of the length of series in a formula:

$$
\begin{aligned}
& \left(\exists \tilde{\phi}: G / G^{(n)} \rightarrow H\right) \tilde{\phi} \circ \omega=\phi \Leftrightarrow \operatorname{dl}(\phi(G)) \leq n, \\
& \left(\exists \tilde{\phi}: G / \gamma_{n}(G) \rightarrow H\right) \tilde{\phi} \circ \omega=\phi \Leftrightarrow \operatorname{cl}(\phi(G)) \leq n-1, \\
& \left(\exists \tilde{\phi}: G / P_{n}(G) \rightarrow H\right) \tilde{\phi} \circ \omega=\phi \Leftrightarrow \operatorname{cl}_{p}(\phi(G)) \leq n .
\end{aligned}
$$

Proof. By induction, we show that, firstly,

$$
\phi\left(G^{(n)}\right)=\phi\left(\left[G^{(n-1)}, \phi^{(n-1)}\right]\right)=\left[\phi\left(G^{(n-1)}\right), \phi\left(G^{(n-1)}\right)\right]=\left[(\phi(G))^{(n-1)},(\phi(G))^{(n-1)}\right]=(\phi(G))^{(n)},
$$

secondly, $\phi\left(\gamma_{n}(G)\right)=\phi\left(\left\lceil G, \gamma_{n-1}(G)\right\rceil\right)=\left\lceil\phi(G), \phi\left(\gamma_{n-1}(G)\right)\right\rceil=\left\lceil\phi(G), \gamma_{n-1}(\phi(G))\right\rceil=\gamma_{n}(\phi(G))$, and finally, $\phi\left(P_{n}(G)\right)=\phi\left(P_{n-1}(G)^{p} \cdot\left[G, P_{n-1}(G)\right]\right)=\phi\left(P_{n-1}(G)\right)^{p} \cdot\left[\phi(G), \phi\left(P_{n-1}(G)\right)\right]$

$$
=P_{n-1}(\phi(G))^{p} \cdot\left[\phi(G), P_{n-1}(\phi(G))\right]=P_{n}(\phi(G)) \text {. }
$$

Now, the claims follow from Corollary 7.1 by observing that $(\phi(G))^{(n)}=1$ iff $\operatorname{dl}(\phi(G)) \leq n$, $\gamma_{n}(\phi(G))=1$ iff $\operatorname{cl}(\phi(G)) \leq n-1$, and $P_{n}(\phi(G))=1$ iff $\operatorname{cl}_{p}(\phi(G)) \leq n$

The following special case is particularly well known. Here we take the commutator subgroup $G^{\prime}$ of $G$ as our charecteristic subgroup, which can either be viewed as the term $\gamma_{2}(G)$ of the lower central series of $G$ or as the term $G^{(1)}$ of the derived series of $G$.

Corollary 7.3. A homomorphism $\phi: G \rightarrow H$ passes through the derived quotient $G / G^{\prime}$ of its source group $G$ if and only if its image $\operatorname{im}(\phi)=\phi(G)$ is abelian.

Proof. Putting $n=1$ in the first statement or $n=2$ in the second statement of Corollary 7.2 we obtain the 
well-known special case that $\phi$ passes through the abelianization $G / G^{\prime}$ if and only if $\phi(G)$ is abelian, which is equivalent to $\operatorname{dl}(\phi(G)) \leq 1$, and also to $\operatorname{cl}(\phi(G)) \leq 1$.

The situation of Corollary 7.3 is visualized in Figure 7.

Using the first part of the proof of Corollary 7.2 we can recognize the behavior of several central series under homomorphisms.

Lemma 7.2. Let $\phi: G \rightarrow H$ be a homomorphism of groups and suppose that $n \geq 1$ is an integer and $p \geq 2$ a prime number. Let $U \leq G$ be a subgroup with image $V=\phi(U) \leq H$.

1) If $n:=\operatorname{dl}(U)$, then

$$
\operatorname{dl}(V) \begin{cases}=n & \Leftrightarrow U^{(n-1)} \nsubseteq \operatorname{ker}(\phi), \\ <n & \Leftrightarrow U^{(n-1)} \leq \operatorname{ker}(\phi) .\end{cases}
$$

2) If $n:=\operatorname{cl}(U)$, then

$$
\operatorname{cl}(V) \begin{cases}=n & \Leftrightarrow \gamma_{n}(U) \nsubseteq \operatorname{ker}(\phi), \\ <n & \Leftrightarrow \gamma_{n}(U) \leq \operatorname{ker}(\phi) .\end{cases}
$$

3) If $n:=\mathrm{cl}_{p}(U)$, then

$$
\operatorname{cl}_{p}(V) \begin{cases}=n & \Leftrightarrow P_{n-1}(U) \nsubseteq \operatorname{ker}(\phi), \\ <n & \Leftrightarrow P_{n-1}(U) \leq \operatorname{ker}(\phi) .\end{cases}
$$

Proof. 1) Let $n:=\operatorname{dl}(U)$, then $U^{(n-1)}>U^{(n)}=1$ and

$V^{(n-1)}=\phi(U)^{(n-1)}=\phi\left(U^{(n-1)}\right) \geq \phi\left(U^{(n)}\right)=\phi(U)^{(n)}=V^{(n)}=1$. Consequently, we have $\operatorname{dl}(V)=n$ if

$U^{(n-1)} \nsubseteq \operatorname{ker}(\phi)$ and $\operatorname{dl}(V)<n$ if $U^{(n-1)} \leq \operatorname{ker}(\phi)$.

2) Let $n:=\operatorname{cl}(U)$, then $\gamma_{n}(U)>\gamma_{n+1}(U)=1$ and

$\gamma_{n}(V)=\gamma_{n}(\phi(U))=\phi\left(\gamma_{n}(U)\right) \geq \phi\left(\gamma_{n+1}(U)\right)=\gamma_{n+1}(\phi(U))=\gamma_{n+1}(V)=1$. Thus, we have $\mathrm{cl}(V)=n$ if

$\gamma_{n}(U) \nsubseteq \operatorname{ker}(\phi)$ and $\operatorname{cl}(V)<n$ if $\gamma_{n}(U) \leq \operatorname{ker}(\phi)$.

3) Let $n:=\operatorname{cl}_{p}(U)$, then $P_{n-1}(U)>P_{n}(U)=1$ and

$P_{n-1}(V)=P_{n-1}(\phi(U))=\phi\left(P_{n-1}(U)\right) \geq \phi\left(P_{n}(U)\right)=P_{n}(\phi(U))=P_{n}(V)=1$. Therefore, we have $\mathrm{cl}_{p}(V)=n$ if $P_{n-1}(U) \nsubseteq \operatorname{ker}(\phi)$ and $\mathrm{cl}_{p}(V)<n$ if $P_{n-1}(U) \leq \operatorname{ker}(\phi)$.

\section{A.5. Application to Automorphisms}

Corollary 7.4. (Induced automorphism)

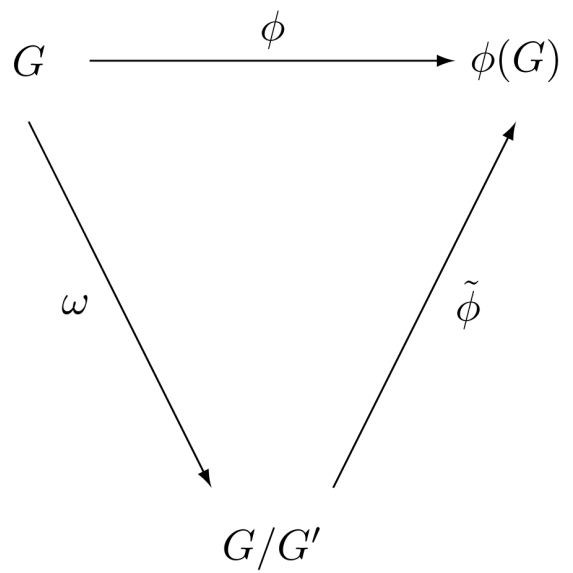

Figure 7. Homomorphism $\phi$ passing through the derived quotient. 
Let $\phi: G \rightarrow H$ be an epimorphism of groups, $\phi(G)=H$, and assume that $\sigma \in \operatorname{Aut}(G)$ is an automorphism of $G$.

1) There exists an induced epimorphism $\hat{\sigma}: H \rightarrow H$ such that $\hat{\sigma} \circ \phi=\phi \circ \sigma$, if and only if $\sigma(\operatorname{ker}(\phi)) \leq \operatorname{ker}(\phi)$, resp. $\operatorname{ker}(\phi) \leq \sigma^{-1}(\operatorname{ker}(\phi))$.

2) The induced epimorphism $\hat{\sigma}$ is also an automorphism of $H, \hat{\sigma} \in \operatorname{Aut}(H)$, if and only if

$$
\sigma(\operatorname{ker}(\phi))=\operatorname{ker}(\phi) \text {. }
$$

In the second statement, $\phi$ is said to have the kernel invariance property (KIP) with respect to $\sigma$.

The situation of Corollary 7.4 is visualized in Figure 8.

Proof. Since $\phi$ is supposed to be an epimorphism, the well-known isomorphism theorem in Remark 7.3 yields a representation of the image $H=\phi(G) \simeq G / \operatorname{ker}(\phi)$ as a quotient.

1) According to Theorem 7.1, the automorphism $\sigma \in \operatorname{Aut}(G)$, simply viewed as a homomorphism $\sigma: G \rightarrow G$, induces a homomorphism $\hat{\sigma}: G / \operatorname{ker}(\phi) \rightarrow G / \operatorname{ker}(\phi)$ if and only if $\sigma(\operatorname{ker}(\phi)) \leq \operatorname{ker}(\phi)$. Since $\sigma$ is an epimorphism, $\hat{\sigma}$ is also an epimorphism with kernel $\operatorname{ker}(\hat{\sigma})=\sigma^{-1}(\operatorname{ker}(\phi)) / \operatorname{ker}(\phi)$.

2) Finally, $\operatorname{ker}(\hat{\sigma})=1 \Leftrightarrow \sigma^{-1}(\operatorname{ker}(\phi))=\operatorname{ker}(\phi) \Leftrightarrow \operatorname{ker}(\phi)=\sigma(\operatorname{ker}(\phi))$.

Remark 7.4. If $\operatorname{ker}(\phi)$ is a characteristic subgroup of $\mathrm{G}$, then Corollary 7.4 makes sure that any automorphism $\sigma \in \operatorname{Aut}(G)$ induces an automorphism $\hat{\sigma} \in \operatorname{Aut}(H)$, where $H=\phi(G) \simeq G / \operatorname{ker}(\phi)$. The reason is that, by definition, a characteristic subgroup of $\mathrm{G}$ is invariant under any automorphism of $\mathrm{G}$.

$\mathbf{P}$ 7.5. We conclude this section with a statement about GI-automorphisms (generator-inverting automorphisms) which have been introduced by Boston, Bush and Hajir ([40], Dfn.2.1). The proof requires results of Theorem 7.1, Corollary 7.4, and Corollary 7.2.

Theorem 7.2. (Induced generator-inverting automorphism)

Let $\phi: G \rightarrow H$ be an epimorphism of groups with $\phi(G)=H$, and assume that $\sigma \in \operatorname{Aut}(G)$ is an automorphism satisfying the KIP $\sigma(\operatorname{ker}(\phi))=\operatorname{ker}(\phi)$, and thus induces an automorphism $\hat{\sigma} \in \operatorname{Aut}(H)$.

If $\sigma$ is generator-inverting, that is,

$$
\sigma(x) G^{\prime}=x^{-1} G^{\prime} \text { for all } x \in G,
$$

then $\hat{\sigma}$ is also generator-inverting, that is, $\hat{\sigma}(y) H^{\prime}=y^{-1} H^{\prime}$ for all $y \in H$.

Proof. According to Corollary 7.4,

$\sigma: G \rightarrow G$ induces an automorphism $\hat{\sigma}: H \rightarrow H$, since $\sigma(\operatorname{ker}(\phi))=\operatorname{ker}(\phi)$.

Two applications of the Remark 7.4 after Corollary 7.4, yield:

$\sigma: G \rightarrow G$ induces an automorphism $\tilde{\sigma}: G / G^{\prime} \rightarrow G / G^{\prime}$, since $G^{\prime}$ is characteristic in $G$, and

$\hat{\sigma}: H \rightarrow H$ induces an automorphism $\bar{\sigma}: H / H^{\prime} \rightarrow H / H^{\prime}$, since $H^{\prime}$ is characteristic in $H$.

Using Theorem 7.1 and the first part of the proof of Corollary 7.2, we obtain:

$\phi: G \rightarrow H$ induces an epimorphism $\tilde{\phi}: G / G^{\prime} \rightarrow H / H^{\prime}$, since $\phi\left(G^{\prime}\right)=\phi(G)^{\prime}=H^{\prime}$.

The actions of the various induced homomorphisms are given by

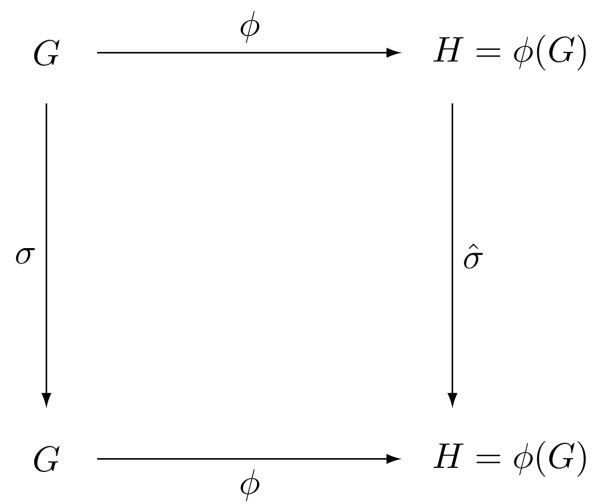

Figure 8. Induced automorphism $\hat{\sigma}$. 
$\hat{\sigma}(\phi(x))=\phi(\sigma(x))$ for $x \in G$,

$\tilde{\sigma}\left(x G^{\prime}\right)=\sigma(x) G^{\prime}$ for $x \in G$,

$\bar{\sigma}\left(y H^{\prime}\right)=\hat{\sigma}(y) H^{\prime}$ for $y \in H$, and

$\tilde{\phi}\left(x G^{\prime}\right)=\phi(x) H^{\prime}$ for $x \in G$.

Finally, combining all these formulas and expressing $H \ni y=\phi(x)$ for a suitable $x \in G$, we see that $\tilde{\sigma}\left(x G^{\prime}\right)=\sigma(x) G^{\prime}=x^{-1} G^{\prime}$ implies the required relation for $\bar{\sigma}\left(y H^{\prime}\right)=\hat{\sigma}(y) H^{\prime}$ :

$$
\hat{\sigma}(y) H^{\prime}=\hat{\sigma}(\phi(x)) H^{\prime}=\phi(\sigma(x)) H^{\prime}=\tilde{\phi}\left(\sigma(x) G^{\prime}\right)=\tilde{\phi}\left(x^{-1} G^{\prime}\right)=\phi\left(x^{-1}\right) H^{\prime}=\phi(x)^{-1} H^{\prime}=y^{-1} H^{\prime}
$$

\section{A.6. Functorial Properties}

P 7.6. The mapping $F: \phi \mapsto F(\phi)=\tilde{\phi}$ which maps a homomorphism of one category to an induced homomorphism of another category can be viewed as a functor $\mathrm{F}$.

In the special case of induced homomorphisms $\tilde{\phi}$ between quotient groups, we define the domain of the functor $F$ as the following category $\mathcal{G}_{n}$.

The objects of the category are pairs $(G, U)$ consisting of a group $G$ and a normal subgroup $U \unlhd G$,

$$
\operatorname{Obj}\left(\mathcal{G}_{n}\right)=\{(G, U) \mid G \in \operatorname{Obj}(\mathcal{G}), U \unlhd G\} .
$$

For two objects $(G, U),(H, V) \in \operatorname{Obj}\left(\mathcal{G}_{n}\right)$, the set of morphisms $\operatorname{Mor}_{\mathcal{G}_{n}}((G, U),(H, V))$ consists of homomorphisms $\phi: G \rightarrow H$ such that $\phi(U) \leq V$, briefly written as arrows $\phi:(G, U) \rightarrow(H, V)$,

$$
\operatorname{Mor}_{\mathcal{G}_{n}}((G, U),(H, V))=\left\{\phi \in \operatorname{Mor}_{\mathcal{G}}(G, H) \mid \phi(U) \leq V\right\} .
$$

The functor $F: \mathcal{G}_{n} \rightarrow \mathcal{G}$ from this new category $\mathcal{G}_{n}$ to the usual category $\mathcal{G}$ of groups maps a pair $(G, U) \in \operatorname{Obj}\left(\mathcal{G}_{n}\right)$ to the corresponding quotient group $F((G, U)):=G / U \in \operatorname{Obj}(\mathcal{G})$, and it maps a morphism $\phi \in \operatorname{Mor}_{\mathcal{G}_{n}}((G, U),(H, V))$ to the induced homomorphism $F(\phi):=\tilde{\phi} \in \operatorname{Mor}_{\mathcal{G}}(G / U, H / V)$,

$$
F: \mathcal{G}_{n} \rightarrow \mathcal{G}, F((G, U)):=G / U, F(\phi):=\tilde{\phi} .
$$

Existence and uniqueness of $F(\phi):=\tilde{\phi}$ have been proved in Theorem 7.1 under the assumption that $\phi(U) \leq V$, which is satisfied according to the definition of the arrow $\phi:(G, U) \rightarrow(H, V)$.

The functorial properties, which are visualized in Figure 9, can be expressed in the following form.

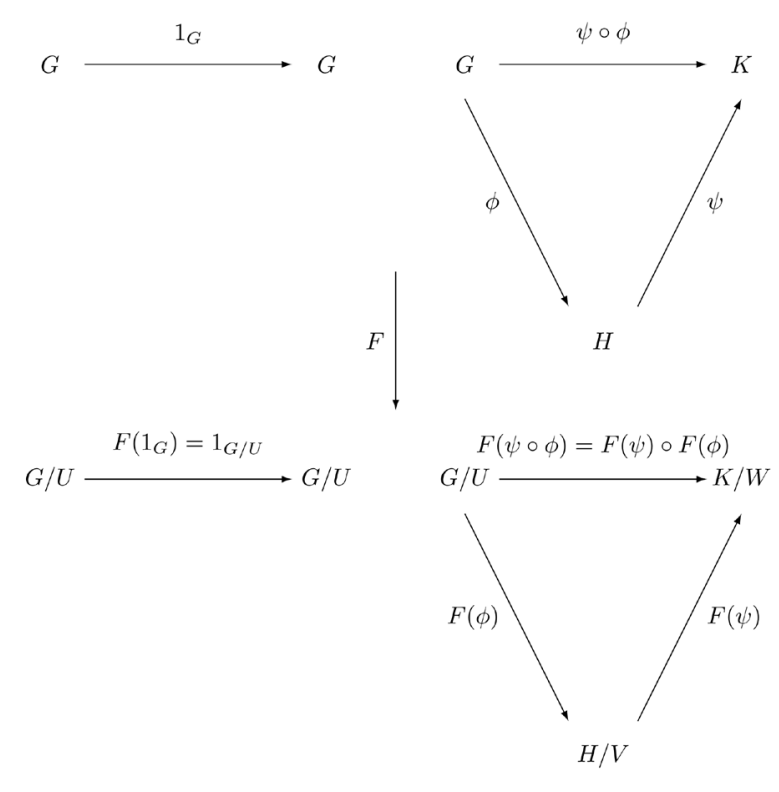

Figure 9. Functorial properties of induced homomorphisms. 
Firstly, $F$ maps the identity morphism $1_{G} \in \operatorname{Mor}_{\mathcal{G}_{n}}((G, U),(G, U))$ having the trivial property $1_{G}(U)=U$ to the identity homomorphism

$$
F\left(1_{G}\right)=\tilde{1}_{G}=1_{G / U} \in \operatorname{Mor}_{\mathcal{G}}(G / U, G / U),
$$

and secondly, $F$ maps the compositum $\psi \circ \phi \in \operatorname{Mor}_{\mathcal{G}_{n}}((G, U),(K, W))$ of two morphisms

$\phi \in \operatorname{Mor}_{\mathcal{G}_{n}}((G, U),(H, V))$ and $\psi \in \operatorname{Mor}_{\mathcal{G}_{n}}((H, V),(K, W))$, which obviously enjoys the required property

$$
(\psi \circ \phi)(U)=\psi(\phi(U)) \leq \psi(V) \leq W,
$$

to the compositum

$$
F(\psi \circ \phi)=(\psi \circ \phi)^{\sim}=\tilde{\psi} \circ \tilde{\phi}=F(\psi) \circ F(\phi) \in \operatorname{Mor}_{\mathcal{G}}(G / U, K / W)
$$

of the induced homomorphisms in the same order.

The last fact shows that $F$ is a covariant functor. 